\title{
Effects of metallicity, star-formation conditions, and evolution in $B$ and Be stars ${ }^{\star}$
}

\section{Small Magellanic Cloud, field of NGC 330}

\author{
C. Martayan ${ }^{1}$, Y. Frémat ${ }^{2}$, A.-M. Hubert ${ }^{1}$, M. Floquet $^{1}$, J. Zorec $^{3}$, and C. Neiner ${ }^{1}$ \\ 1 GEPI, UMR 8111 du CNRS, Observatoire de Paris-Meudon, 92195 Meudon Cedex, France \\ e-mail: christophe .martayan@obspm. fr \\ 2 Royal Observatory of Belgium, 3 avenue circulaire, 1180 Brussels, Belgium \\ 3 Institut d'Astrophysique de Paris (IAP), 98bis boulevard Arago, 75014 Paris, France
}

Received 23 February 2006 / Accepted 20 September 2006

\section{ABSTRACT}

\begin{abstract}
Aims. We search for the effects of metallicity on B and Be stars in the Small and Large Magellanic Clouds (SMC and LMC) and in the Milky Way (MW), by extending our previous analysis of B and Be star populations in the LMC to the SMC. The rotational velocities of massive stars and the evolutionary status of Be stars are examined with respect to their environments.

Methods. Spectroscopic observations of hot stars belonging to the young cluster SMC-NGC 330 and its surrounding region were obtained with the VLT-GIRAFFE facilities in MEDUSA mode. We determined fundamental parameters for B and Be stars with the GIRFIT code, taking the effect of fast rotation and the age of observed clusters into account. We compared the mean $V \sin i$ obtained by spectral type- and mass-selection for field and cluster B and Be stars in the SMC with the one in the LMC and MW.

Results. We find that (i) B and Be stars rotate faster in the SMC than in the LMC and in the LMC than in the MW; (ii) at a given metallicity, Be stars begin their main sequence life with a higher initial rotational velocity than B stars. Consequently, only a fraction of the B stars that reach the ZAMS with a sufficiently high initial rotational velocity can become Be stars; (iii) the distributions of initial rotational velocities at the ZAMS for Be stars in the SMC, LMC, and MW are mass- and metallicity-dependent; (iv) the angular velocities of B and Be stars are higher in the SMC than in the LMC and MW; (v) in the SMC and LMC, massive Be stars appear in the second part of the main sequence, in contrast to massive Be stars in the MW.
\end{abstract}

Key words. stars: early-type - stars: emission-line, Be - galaxies: Magellanic Clouds - stars: fundamental parameters stars: evolution - stars: rotation

\section{Introduction}

The origin of the Be phenomenon has given rise to long debates. Whether it is linked to stellar evolution or initial formation conditions remains a major issue. Thus, finding out differences in the physical properties of $\mathrm{B}$ and Be stars populations belonging to environments with different metallicity could provide new clues to understanding the Be phenomenon.

To investigate the influence of metallicity, star-formation conditions, and stellar evolution on the Be phenomenon, we have undertaken an exhaustive study of B and Be stars belonging to young clusters or the field of the Small and Large Magellanic Clouds (SMC and LMC), because these galaxies have a lower metallicity than the Milky Way (MW). For this purpose we made use of the new FLAMES-GIRAFFE instrumentation installed at the VLT-UT2 at ESO, which is particularly well-suited, in MEDUSA mode, to obtaining the high quality spectra of large samples needed to study stellar populations. In Martayan et al. (2006a, hereafter M06), we reported on the identification of $177 \mathrm{~B}$ and Be stars belonging to the young cluster LMCNGC 2004 and its surrounding region. In Martayan et al. (2006b, hereafter Paper I), we determined fundamental parameters of a large fraction of the sample in the LMC, taking into account

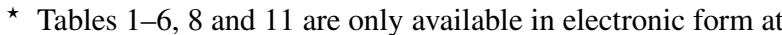
http://www. aanda.org rotational effects (stellar flattening, gravitational darkening) when appropriate. We then investigated the effects of metallicity on rotational velocities. We concluded that Be stars begin their life on the main sequence (MS) with a higher initial velocity than B stars. Moreover, this initial velocity is sensitive to the metallicity. Consequently, only a fraction of the B stars that reach the ZAMS with a sufficiently high initial rotational velocity can become Be stars. However, no clear influence of metallicity on the rotational velocity of B stars was found.

The present paper deals with a large sample of $\mathrm{B}$ and $\mathrm{Be}$ stars in the SMC, which has a lower metallicity than the LMC. With the determination of fundamental parameters and the study of the evolutionary status, we aim at confirming and enlarging our results derived from the study of B and Be stars in Martayan et al. (2005a) and from the LMC (Paper I).

\section{Observations}

This work makes use of spectra obtained with the multifibre VLT-FLAMES/GIRAFFE spectrograph in Medusa mode (131 fibres) at medium resolution $(R=6400)$ in setup LR02 (396.4-456.7 nm). Observations (ESO runs 72.D-0245A and 72.D-0245C) were carried out in the young cluster SMCNGC330 and in its surrounding field, as part of the Guaranteed Time Observation programmes of the Paris Observatory 


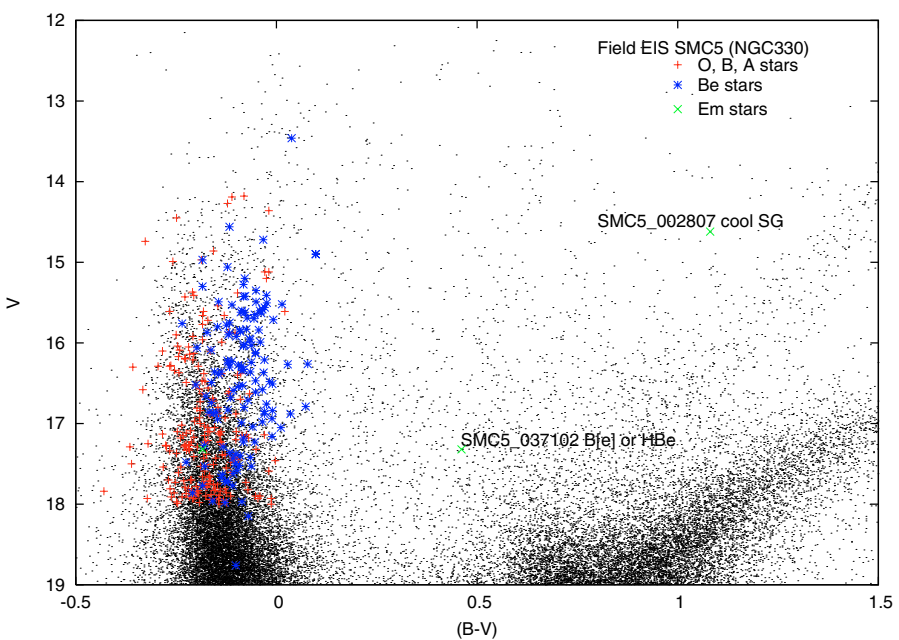

Fig. 1. $V$ versus $(B-V)$ colour diagram from EIS photometry in the EIS SMC 5 field. The "." symbols correspond to all stars in this field. "*" show the Be stars, "+" the O-B-A stars and " $x$ " the other emissionline stars in the sample.

(P.I.: F. Hammer). The observed fields $\left(25^{\prime}\right.$ in diameter $)$ are centred at $\alpha(2000)=00 \mathrm{~h} 55 \min 15 \mathrm{~s}, \delta(2000)=-72^{\circ} 20^{\prime} 00^{\prime \prime}$ and $\alpha(2000)=00 \mathrm{~h} 55 \min 25 \mathrm{~s}, \delta(2000)=-72^{\circ} 23^{\prime} 30^{\prime \prime}$. Besides the young cluster NGC 330, this field contains several highdensity groups of stars (NGC 306, NGC 299, OGLE-SMC99, OGLE-SMC109, H86 145, H86 170, Association [BS95]78, Association SMC ASS39). Note that we corrected the coordinates of NGC 299 given in Simbad (CDS) with EIS coordinates (Momany et al. 2001). Spectra were obtained on October 21, 22, and 23, 2003 and September 9 and 10, 2004; on those dates, the heliocentric velocity was 12 in October and $7 \mathrm{~km} \mathrm{~s}^{-1}$ in September. The strategy and conditions of observations, as well as the spectra-reduction procedure, are described in M06. A sample of 346 stars was observed within the two observing runs. Since the $V$ magnitude of the selected targets ranges from 13.5 to $18.8 \mathrm{mag}$, the integration time varies between $1 \mathrm{~h}$ and $2 \mathrm{~h}$. However, as the seeing was not optimal during the first run, the $S / N$ ratio is only about 50 on average, with individual values ranging from 20 to 130 .

We pre-selected 11544 B-type star candidates with $14 \leq$ $V \leq 18$ and a colour index $B-V<0.35$, among the 192437 stars listed in the EIS SMC5 field by the EIS team (Momany et al. $2001)$, keeping in mind the intrinsic value $E(B-V)=0.08$ (Keller et al. 1999) for the SMC. We then observed three fields with VLT-FLAMES/GIRAFFE. Since 130 stars can be observed for each field maximum, we collected data for 346 objects among the 5470 B-type star candidates located in these fields for the selected magnitude range. The ratio of observed to observable B-type stars in the GIRAFFE fields is thus $6.3 \%$. This represents a statistically significant sample. From the observations we confirm the B spectral type for 333 of the 346 stars. Of the remaining 13 objects, 4 are O stars, 6 are A stars, 1 is a cold supergiant, 1 a planetary nebula, and 1 a $\mathrm{HB}[\mathrm{e}]$. The 333 B-type objects further include 131 Be stars.

The $V$ versus $B-V$ colour diagram (Fig. 1), derived from EIS photometry (Momany et al. 2001), shows the O, B, A, and Be stars in our sample compared to all the stars in the EISSMC 5 field.

\section{Determining fundamental parameters}

As in Paper I we make use of the GIRFIT least-square procedure (Frémat et al. 2006) to derive the fundamental parameters: effective temperature $\left(T_{\text {eff }}\right)$, surface gravity $(\log g)$, projected rotational velocity $(V \sin i)$, and radial velocity $(\mathrm{RV})$. This procedure fits the observations with theoretical spectra interpolated in a grid of stellar fluxes computed with the SYNSPEC programme and from model atmospheres calculated with TLUSTY (Hubeny \& Lanz (1995, see references therein) or/and with ATLAS9 (Kurucz 1993; Castelli et al. 1997). The grid of model atmospheres we used to build the GIRFIT input of stellar fluxes was obtained in the same way as in our LMC study, but for the metallicity of the SMC.

The metallicities of the model atmospheres were chosen to be as close as possible to the NGC330 average value, $[m / H]=-0.6\left(\right.$ where $\left.[m / H]=\log (m / H)_{\text {SMC }}-\log (m / H)_{\odot}\right)$, estimated from Jasniewicz \& Thévenin (1994). The Kurucz and OSTAR 2002 models we used are therefore those calculated with a $[\mathrm{m} / \mathrm{H}]$ close to -0.6 . Finally, the complete input flux grid was built assuming the averaged element abundances derived by Jasniewicz \& Thévenin (1994) for C, Mg, Ca, Ti, Cr, Mn, and Fe. The other elements, except hydrogen and helium, were assumed to be underabundant by -0.6 dex relative to the Sun.

It is worth noting that GIRFIT does not include the effects of fast rotation. Therefore, for rapidly rotating stars, we needed to correct the stellar parameters with the FASTROT computer code (Frémat et al. 2005) assuming a solid-body-type rotation. We then obtained the "parent non-rotating counterpart" (pnrc; see Frémat et al. 2005) stellar parameters $\left(T_{\mathrm{eff}}^{\mathrm{o}}, \log g_{\mathrm{o}}, V \sin i^{\text {true }}\right)$ for a given $\Omega / \Omega_{\mathrm{c}}$.

For a more detailed description of the grid of model atmospheres we used, the fitting criteria we adopt in the GIRFIT procedure, and the correction for fast rotation we applied to the fundamental parameters of Be stars, we refer the reader to Paper I (Sect. 3).

Finally, we determined the spectral classification of each star with two methods. The calibration we established to estimate these spectral types is described in Paper I. The agreement between the two methods is not as good as for the stars observed in the LMC (Paper I), because the observations for the SMC have a lower $S / N$.

\section{Stellar parameters of the sample stars}

In this section we present the stellar parameters and spectral classification we obtained for O-B-A and Be stars.

\subsection{Fundamental parameters of $O-B-A$ stars}

Early-type stars that do not show intrinsic emission lines in their spectra and have not been detected as spectroscopic binaries are listed in Table 1, sorted by their EIS catalogue number. The fundamental parameters $T_{\text {eff }}, \log g, V \sin i$, and $R V$ obtained by fitting the observed spectra, as well as the spectral classification deduced on one hand from $T_{\text {eff }}-\log g$ plane calibration (CFP determination, see Paper I) and on the other hand from equivalent width diagrams (CEW determination, see Paper I), are reported in Table 1 . The heliocentric velocities $\left(7\right.$ and $12 \mathrm{~km} \mathrm{~s}^{-1}$ ) have been subtracted from the radial velocities.

To derive the luminosity, mass, and radius of $\mathrm{O}, \mathrm{B}$, and A stars from their fundamental parameters, we interpolated in the HR-diagram grids (Schaller et al. 1992) calculated for the 
SMC metallicity $(Z=0.001$; Maeder et al. 1999 and references therein) and for stars without rotation.

We estimated the mean radius, mean mass, and mean $V \sin i$ in various mass bins (e.g. $5<M<7 M_{\odot}, 7<M<9 M_{\odot}$, etc). We then obtained a mean equatorial velocity for a random angle distribution using formulae published in Chauville et al. (2001) and Paper I.

For B stars, $\langle V \sin i\rangle$ is close to $160 \mathrm{~km} \mathrm{~s}^{-1}$, thus $V_{\mathrm{e}} / V_{\mathrm{c}} \simeq 43 \%$ and $\Omega / \Omega_{\mathrm{c}} \simeq 58 \%$. As the effects of fast rotation on the spectra are only significant for $\Omega / \Omega_{\mathrm{c}}>60 \%$ (Frémat et al. 2005), we not need to correct the fundamental parameters of B stars for fast rotation effects. This justifies the use of non-rotating models. Although some B stars do have a high $V \sin i\left(>350 \mathrm{~km} \mathrm{~s}^{-1}\right)$, the accuracy of the parameters determination is generally low for these stars, and thus we decided not to introduce corrections. Since the value of the averaged $\Omega / \Omega_{\mathrm{c}}$ is at the limit at which the spectroscopic effects of fast rotation appear, we however expect that a significant part of the B stars in the sample will apparently be more evolved due to gravitational darkening.

The obtained luminosity, mass, radius, and age of most O, B, and A stars of the sample are given in Table 2. The position of these stars in the HR diagram is shown in Fig. 2.

\subsection{Fundamental parameters of Be stars}

\subsubsection{Apparent fundamental parameters}

The sample (131 Be stars) includes 41 known Be stars from Keller et al. (1999) and from Grebel et al. (1992), for which the $\mathrm{H} \alpha$ emissive character has been confirmed in this work, and 90 new Be stars. Three $\mathrm{H} \alpha$ emission line stars mentioned in Keller et al. (1999) are not Be stars: the star SMC5_2807, or $\mathrm{KWBBe} 044$, is a cool supergiant and a binary; the star SMC5_37102 or KWBBe485 is a possible HB[e]; and the star SMC5_81994, or KWBBe4154, is a planetary nebula.

The apparent fundamental parameters $\left(T_{\mathrm{eff}}^{\text {app. }}, \log g_{\text {app. }}\right.$, $V \sin i_{\text {app. }}$, and $R V$ ) we derive for these stars are reported in Table 3. The spectral classification derived from apparent fundamental parameters is also given in the last column of the table. Without correction for fast rotation nearly all Be stars seem to be sub-giants or giants.

The apparent luminosity, mass, radius, and age of Be stars are derived in the same way as for O, B, and A stars (see Sect. 4.1), from their apparent fundamental parameters. The apparent position of Be stars in the HR diagram is shown in Fig. 2, and the corresponding luminosities, masses, and radii are given in Table 4.

\subsubsection{Fundamental parameters corrected for rapid rotation}

The pnrc fundamental parameters $\left(T_{\mathrm{eff}}^{\mathrm{o}}, \log g_{\mathrm{o}}, V \sin i^{\text {true }}\right)$ of Be stars obtained after correction with FASTROT are given in Table 5 for different rotation rates $\Omega / \Omega_{\mathrm{c}}$. We estimated the rotation rate $\Omega / \Omega_{\mathrm{c}}$ to be used for the selection of the most suitable pnrc fundamental parameters of Be stars in the SMC as in Paper I. We obtained $V_{\mathrm{e}} / V_{\mathrm{c}} \simeq 87 \%$ and $\Omega / \Omega_{\mathrm{c}} \simeq 95 \%$ on average.

As previously, but with the pnrc fundamental parameters corresponding to the rotation rate $\Omega / \Omega_{\mathrm{c}}=95 \%$, we derived the luminosity $\log \left(L / L_{\odot}\right)$, mass $M / M_{\odot}$, and radius $R / R_{\odot}$ for Be stars. These parameters are given in Table 6 . After correction for rapid rotation, Be stars globally shift in the HR diagram towards lower luminosity and higher temperature, as illustrated in Fig. 2. It
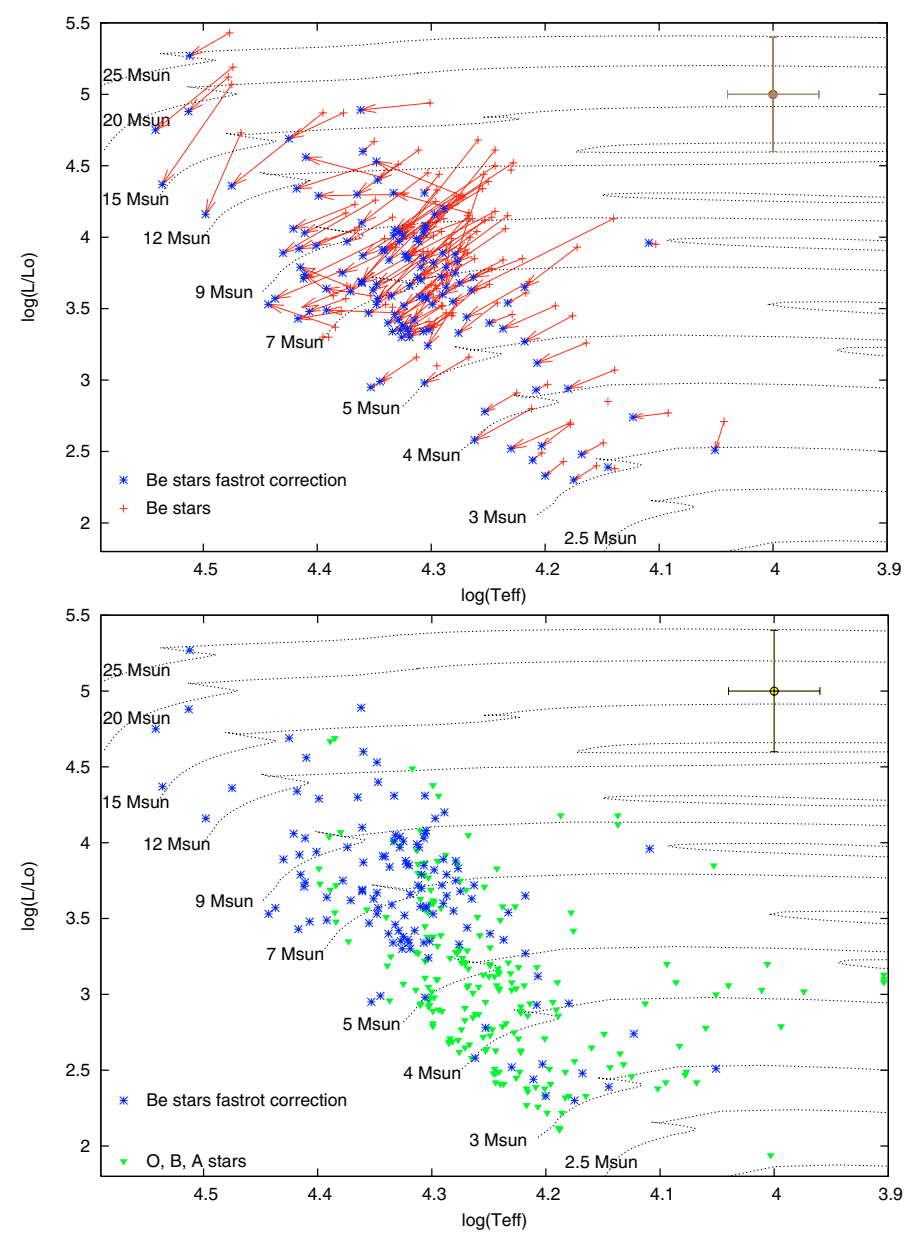

Fig. 2. HR diagrams for the studied B and Be stars. Top: the effects of fast rotation are taken into account with $\Omega / \Omega_{c}=95 \%$ for Be stars. Bottom: B stars and fast rotators (Be stars) corrected for their fast rotation. Common: the adopted metallicity for the SMC is $Z=0.001$. Red "+" represent Be stars with their apparent parameters, blue “*” Be stars corrected with FASTROT with $\Omega / \Omega_{\mathrm{c}}=95 \%$, and green triangles B stars. Typical error bars are shown in the upper right hand corner of the figure. Evolutionary tracks come from Schaller et al. (1992).

clearly demonstrates that Be stars are less evolved than their apparent fundamental parameters would indicate.

\subsubsection{Spectral line saturation}

According to Townsend et al. (2004) and Frémat et al. (2005, Figs. 5 and 6), there may be a saturation effect of the FWHM of spectral lines for the highest angular velocities $\left(\Omega / \Omega_{c}\right)$, which hampers the estimate of $V \sin i$. However, the magnitude of this effect strongly depends on stellar parameters and on the studied line-transitions. Multiple line fitting, as performed in our study, allows therefore reduction of the impact of the saturation (due to the gravitational darkening) and to correct it with FASTROT. This is confirmed by the fact that, for the SMC, we report apparent $\frac{V_{\mathrm{e}}}{V_{\mathrm{c}}}$ ratios that are significantly above the expected limit where saturation should appear (i.e. $\sim 0.80$ ).

\subsection{Characteristics of the sample}

To characterise the sample of stars, we studied the distribution in spectral types, luminosity classes, and masses for stars in clusters and in the field. Note that the method with equivalent 

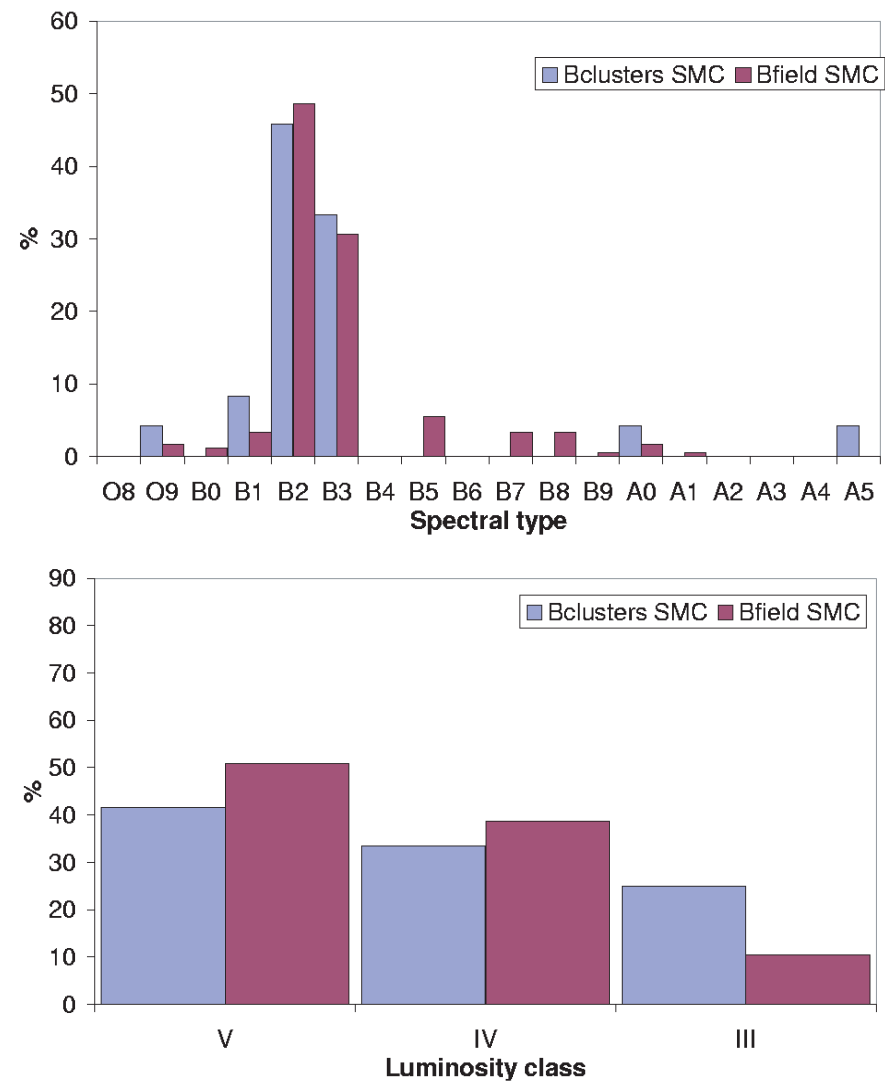

Fig. 3. Spectral type (upper panel) and luminosity class (lower panel) distributions of B-type stars in the sample in the SMC. In common: the blue left bars are for stars in clusters and the red right bars are for stars in fields.

widths (CEW) fails to give a reliable spectral classification for the few hotter (late O) and cooler (B5-A0) stars in the sample. Moreover, for Be stars, the spectral classification is only determined by using the derived apparent fundamental parameters (CFP), since the emission contamination, often present in $\mathrm{H} \gamma$ and in several cases in the He I 4471 line, makes the first method particularly inappropriate for early Be stars.

\subsubsection{Distributions in spectral types and luminosity classes}

We present the distribution of O-B-A stars in Fig. 3 with respect to spectral type and luminosity class. The classification used here is the one obtained from the fundamental parameters determination (CFP determination). The sample contains essentially early B-type stars (B0 to B3) as in the LMC (Paper I), which are mainly dwarfs and subgiants (classes V, IV) in the field, as well as in clusters.

We also present the distribution of Be stars with respect to luminosity class and spectral type, using the classification obtained from the fundamental parameters. We compared the distribution obtained before and after correction of fast rotation effects (Figs. 4 and 5).

As for B stars, the Be stars in our sample generally are early B-type stars (B0 to B3) but are apparently giants and subgiants (classes III, IV). The Be stars corrected for rotation effects appear hotter than apparent fundamental parameters would suggest. In particular, there are more B1-type stars. After fast-rotation treatment, the Be stars in classes III and IV are
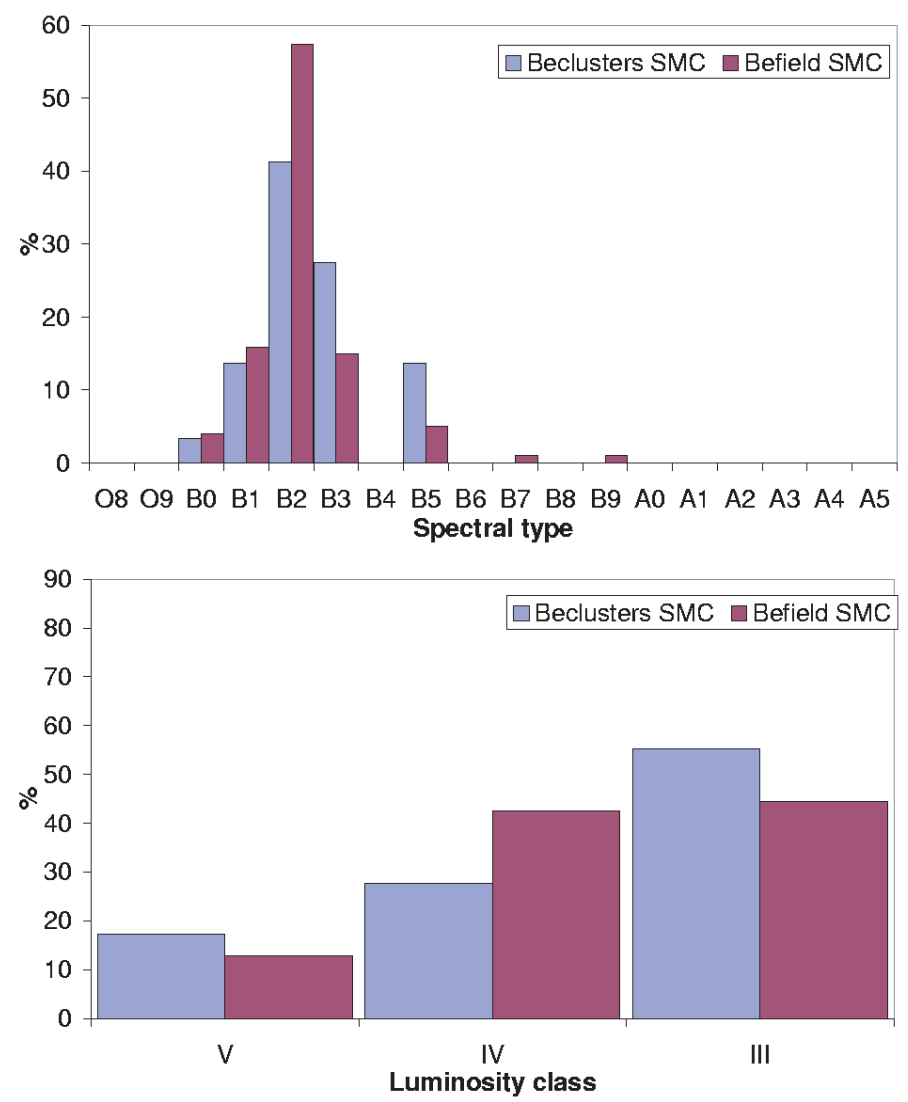

Fig. 4. Apparent spectral type (upper panel) and luminosity class (lower panel) distributions of Be stars in the sample in the SMC. In common: The blue left bars are for stars in clusters and the red right bars are for stars in fields.

redistributed in classes IV and V. However, about $60 \%$ of the Be stars still appear as giants and subgiants as in the LMC (Paper I).

\subsubsection{Distribution in masses}

In addition, we investigated the mass distribution of $\mathrm{B}$ and $\mathrm{Be}$ stars (Fig. 6). The sample shows a distribution peaking around 5-6 and 7-8 $M_{\odot}$ for B and Be stars, respectively. These peaks are reminiscent of those for B and Be stars in LMC's sample (7 and $10 M_{\odot}$ ) as shown in Paper I, but are shifted to lower masses.

\subsubsection{Ages of clusters}

We determined the ages of stars of the field and of several clusters or associations in our observations. For this purpose, we used HR evolutionary tracks (for non-rotating stars) for the stars of the sample unaffected by rapid rotation and for Be stars corrected for the effects of fast rotation with $\Omega / \Omega_{\mathrm{c}}=95 \%$. For the cluster NGC 330, we obtain $\log (t)=7.5 \pm 0.2$, which is in excellent agreement with the value found photometrically by OGLE $(7.5 \pm 0.1$, see Pietrzyński \& Udalski 1999), while Chiosi et al. 2006 found $\log (t)=8.0$. For the clusters OGLESMC99 and OGLE-SMC109, we find $\log (t)=7.8 \pm 0.2$ and $\log (t)=7.9 \pm 0.2$, to be compared with $\log (t)=7.6 \pm 0.2$ and $\log (t)=7.7 \pm 0.1$, respectively (Pietrzyński \& Udalski 1999), with 7.3 and 7.4 respectively from Chiosi et al. (2006), and with 8.1 and 7.8 respectively from Rafelski \& Zaritsky (2005) for a metallicity $Z=0.001$. In the same way, we determine the age of other clusters: for NGC $299 \log (t)=7.8 \pm 0.2$ and NGC 306 

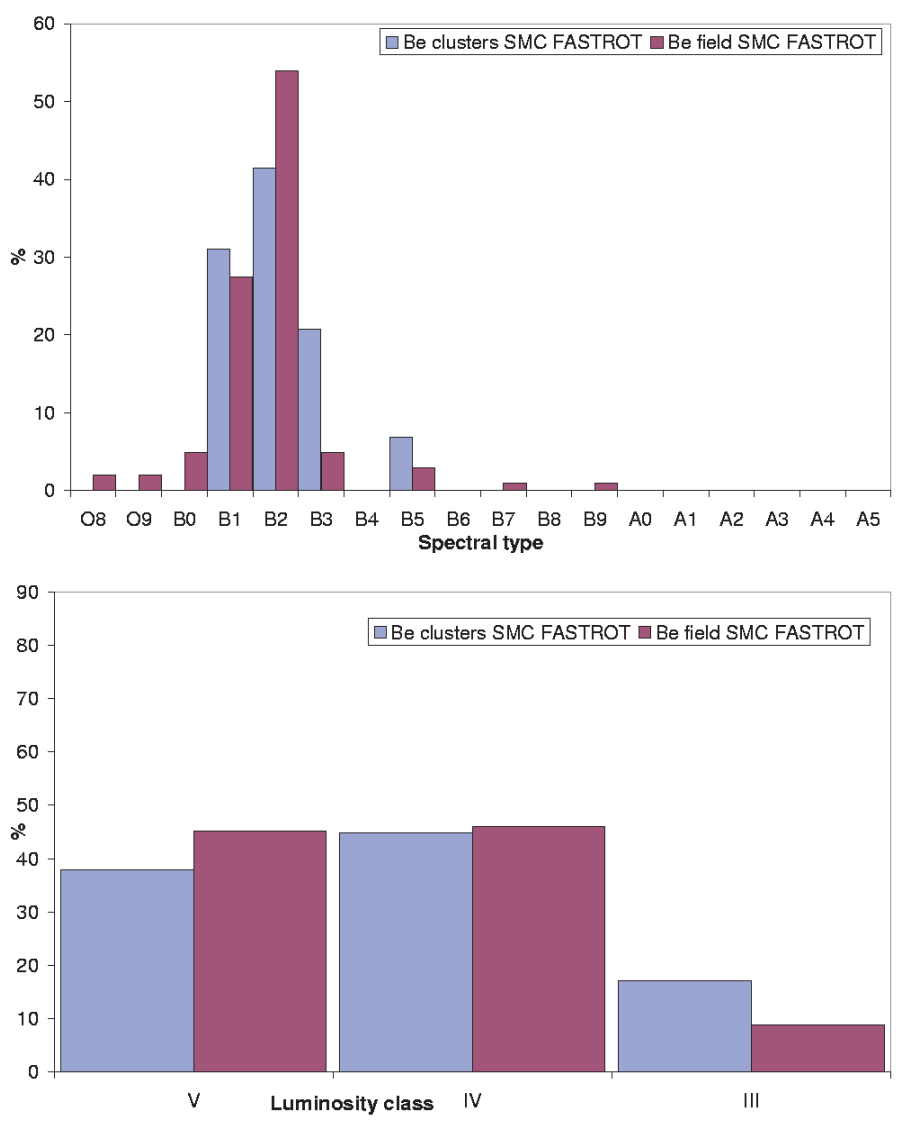

Fig. 5. Corrected spectral type (upper panel) and luminosity class (lower panel) distributions of Be stars after fast rotation treatment in the sample in the SMC. In common: The blue left bars are for stars in clusters and the red right bars are for stars in fields.

$\log (t)=7.9 \pm 0.2$ to be compared with the values from Rafelski \& Zaritsky (2005): 7.9 and 8.5 respectively. Our values are thus generally in good agreement with OGLE and other determinations. As for the LMC, these comparisons validate our method in determining the ages for clusters.

\section{Rotational velocity and metallicity: results and discussion}

We compared the $V \sin i$ values obtained for B and Be stars in the SMC to those obtained for the LMC (Paper I) and given in the literature for the MW. However, the latter generally did not take fast rotation effects into account in determining fundamental parameters. Therefore, to allow the comparison with the MW, we report on the apparent rotational velocity in the case of rapid rotators in the SMC and LMC.

\subsection{V sin i for the SMC in comparison with the $L M C$ and MW}

For the same reasons as in Paper I, we cannot directly compare the mean $V \sin i$ values of the sample in the SMC with values in the LMC and in the MW, because they are affected by ages and evolution, mass function of samples, etc. We must therefore select B and Be stars in the same range of spectral types and luminosity classes or of masses (when they are known) and ages for samples in the SMC, LMC, and MW.

To investigate the effect of metallicity and age on the rotational velocity, we first compared the mean $V \sin i$ of $\mathrm{B}$ and $\mathrm{Be}$ stars either in the field or in clusters in the SMC to the ones in

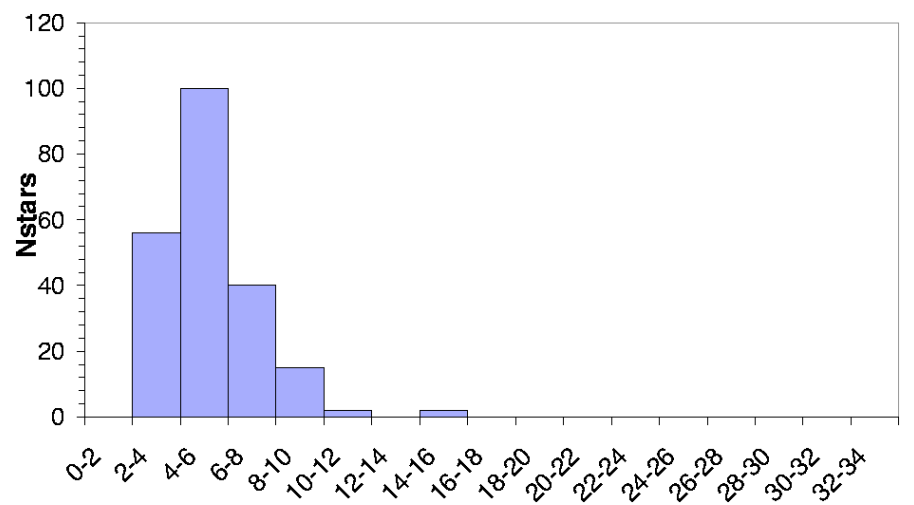

$M$ in $\mathbf{M}_{\odot}$

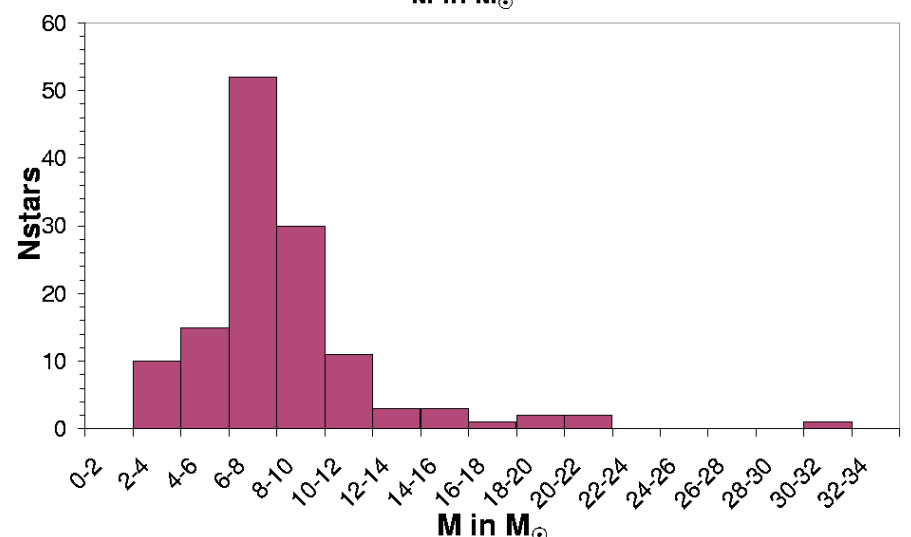

Fig. 6. Mass distribution of B (upper panel) and apparent mass distribution of Be stars (lower panel) in the sample in the SMC.

the LMC and MW. Then, we compared the rotational velocity of $\mathrm{B}$ and Be stars in field versus clusters in the SMC. We used the same selection criteria as those for the LMC and MW described in Paper I: we selected stars with spectral type ranging from B1 to B3 and luminosity classes from V to III. For reference studies in the LMC and MW, we used the same as those mentioned in Paper I (see Table 10 therein). The values are reported in Table 7. As in Paper I, we recall that all suspected binaries, shown in Martayan et al. (2005b) are removed from the statistics of the subsequent sections.

\subsubsection{Field $B$ and Be stars}

The comparison of $V \sin i$ in the SMC, the LMC and the MW for $\mathrm{B}$ and $\mathrm{Be}$ stars in the field are presented in Table 7 and Fig. 7 (upper panel). In the figure the range of stellar ages is reported as the dispersion in age. For samples with an unknown age, we adopt the duration of the main sequence for a $7 M_{\odot}$ star as error bar, which highly overestimates the age uncertainty. The curves show the evolutionary tracks of rotational velocity during the main sequence for different initial velocities for a $7 M_{\odot}$ star, which corresponds to the maximum of the mass function of the B-star sample. These curves were obtained as described in Paper I (Sect. 5.2).

As for the LMC, we show that the samples in the SMC contain a sufficient number of elements for the statistics to be relevant and give an average $V \sin i$ not biased by inclination effects. We completed the statistical study by the Student's t-test (Table 8) in order to know whether the differences observed between samples in the SMC, LMC, and MW are significant. 
Table 7. Comparison of mean rotational velocities for B and Be stars with spectral types B1-B3 and luminosity classes from V to III in the SMC, LMC, and MW. *Values in brackets represent the number of stars in the samples.

\begin{tabular}{|c|c|c|c|c|c|}
\hline From & & Field B stars & Field Be stars & Clusters B stars & Clusters Be stars \\
\hline this study & SMC & $159 \pm 20(147)^{*}$ & $318 \pm 30(87)$ & $163 \pm 18(19)$ & $264 \pm 30(25)$ \\
\hline Paper I & LMC & $121 \pm 10(81)$ & $268 \pm 30(26)$ & $144 \pm 20(10)$ & $266 \pm 30(19)$ \\
\hline Paper I & LMC Keller (2004) & $112 \pm 50(51)$ & & $146 \pm 50(49)$ & \\
\hline Paper I & MW Glebocki et al. (2000) & $124 \pm 10(449)$ & $204 \pm 20(48)$ & & \\
\hline Paper I & MW Levato et al. (2004) & $108 \pm 10(150)$ & & & \\
\hline Paper I & MW Yudin (2001) & & $207 \pm 30(254)$ & & \\
\hline Paper I & MW Chauville et al. (2001) & & $231 \pm 20(56)$ & & \\
\hline Paper I & MW WEBDA $\log (t)<7$ & & & $127 \pm 20(44)$ & $199 \pm 20(8)$ \\
\hline Paper I & MW WEBDA $\log (t) \geq 7$ & & & $149 \pm 20(59)$ & $208 \pm 20(45)$ \\
\hline
\end{tabular}
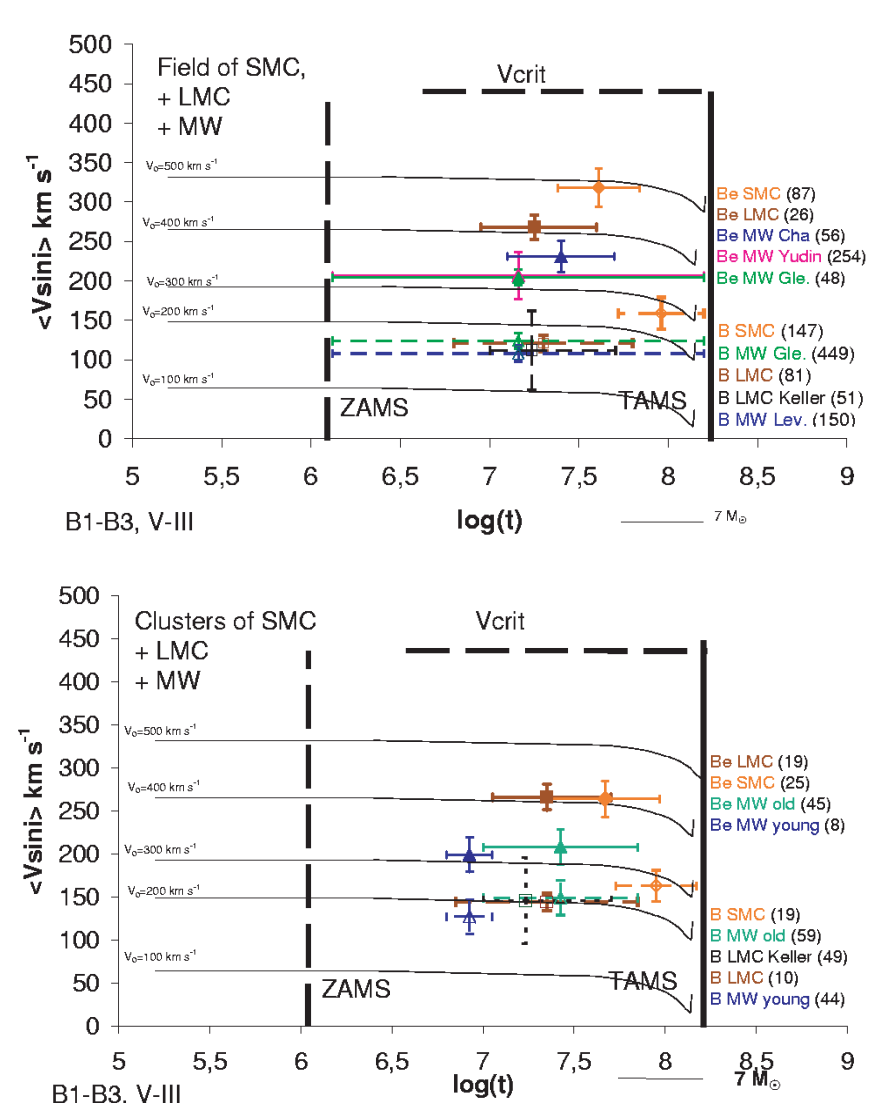

Fig. 7. Comparison of mean $V \sin i$ in the SMC, in the LMC and in the MW. Evolutionary tracks of rotational velocity during the main sequence life are given for different initial velocities for a $7 M_{\odot}$ star. The ZAMS and TAMS are indicated by vertical lines and the critical $V \sin i$ by a horizontal dotted line. The number of stars for each study is given in brackets. The dispersion in ages corresponds to the range of individual stellar ages in the samples (when these ages are known) or to the main sequence lifetime. Upper panel: for field B and Be stars. The considered studies are: for the SMC, this paper; for the LMC, Martayan et al. (2006b) and Keller (2004); for the MW, Cha = Chauville et al. (2001), Yudin (2001), Gle = Glebocki \& Stawikowski (2000), and Lev = Levato \& Grosso (2004). Lower panel: same figure but for clusters. The considered studies are: for the SMC, this paper; for the LMC, Paper I and Keller (2004), and for the clusters in the MW, the WEBDA database.

(i) for field B stars: we find that there is a significant difference between the SMC, the LMC, and the MW. Field B stars in the SMC have a rotational velocity higher than in the LMC and the MW. We recall that the test is not conclusive between B stars in the LMC and the MW (Paper I), because results are different according to the selected study in the MW (Glebocki et al. 2000 or Levato et al. 2004).

(ii) for field $\mathrm{Be}$ stars: there is a slight difference between the SMC and the LMC, and a significant difference between the SMC and the MW. Field Be stars in the SMC have a higher rotational velocity than in the LMC and the MW. We also recall that, from Paper I, field Be stars in the LMC have a rotational velocity higher than in the MW.

\subsubsection{B and Be stars in clusters}

The comparison of $V \sin i$ in the SMC, LMC, and MW for B and Be stars in clusters are presented in Table 7 and Fig. 7 (lower panel). The evolutionary tracks curves are the same as in the upper panel. For the MW, we use the selection we made in Paper I. We distinguish two groups: the younger clusters with $\log (t)<7$ and older clusters with $\log (t) \geq 7$. The age-difference between clusters, taken from WEBDA, gives the age-dispersion reported in the figure. The results concerning B and Be stars in the SMC, LMC, and MW clusters are:

(i) B stars in the SMC and LMC clusters seem to have a similar rotational velocity, as in the MW when interval of similar ages are compared (Paper I).

(ii) for Be stars: We note no difference between Be stars in the SMC and LMC clusters, while there is a significant difference between the LMC and the MW clusters. Be stars rotate more rapidly in the Magellanic Clouds (MC) clusters than the MW clusters. The lack of difference between the SMC and the LMC is probably due to a difference in mass and evolution functions of the stars in the samples (see Sect. 4.3.2 and Paper I, Sect. 4.5.3).

\subsubsection{Comparison between field and clusters}

No significant differences can be found between rotational velocities whether for field versus cluster B stars in the SMC, LMC, and MW or for field versus cluster Be stars in the LMC and the MW. However, a slight trend seems to be present for Be stars in the SMC. Field Be stars seem to rotate faster than cluster Be stars in the SMC. However, note the large error bar on the mean $V \sin i$ value for Be stars in the SMC, which prevents conclusive results between field and clusters.

\subsection{B and Be stars: mass and rotation}

The search for links between metallicity and the rotation of $\mathrm{B}$ and Be stars is also carried out thanks to a selection by masses, which allows a direct comparison with theoretical tracks of the rotational velocities. To obtain sub-samples in the most 


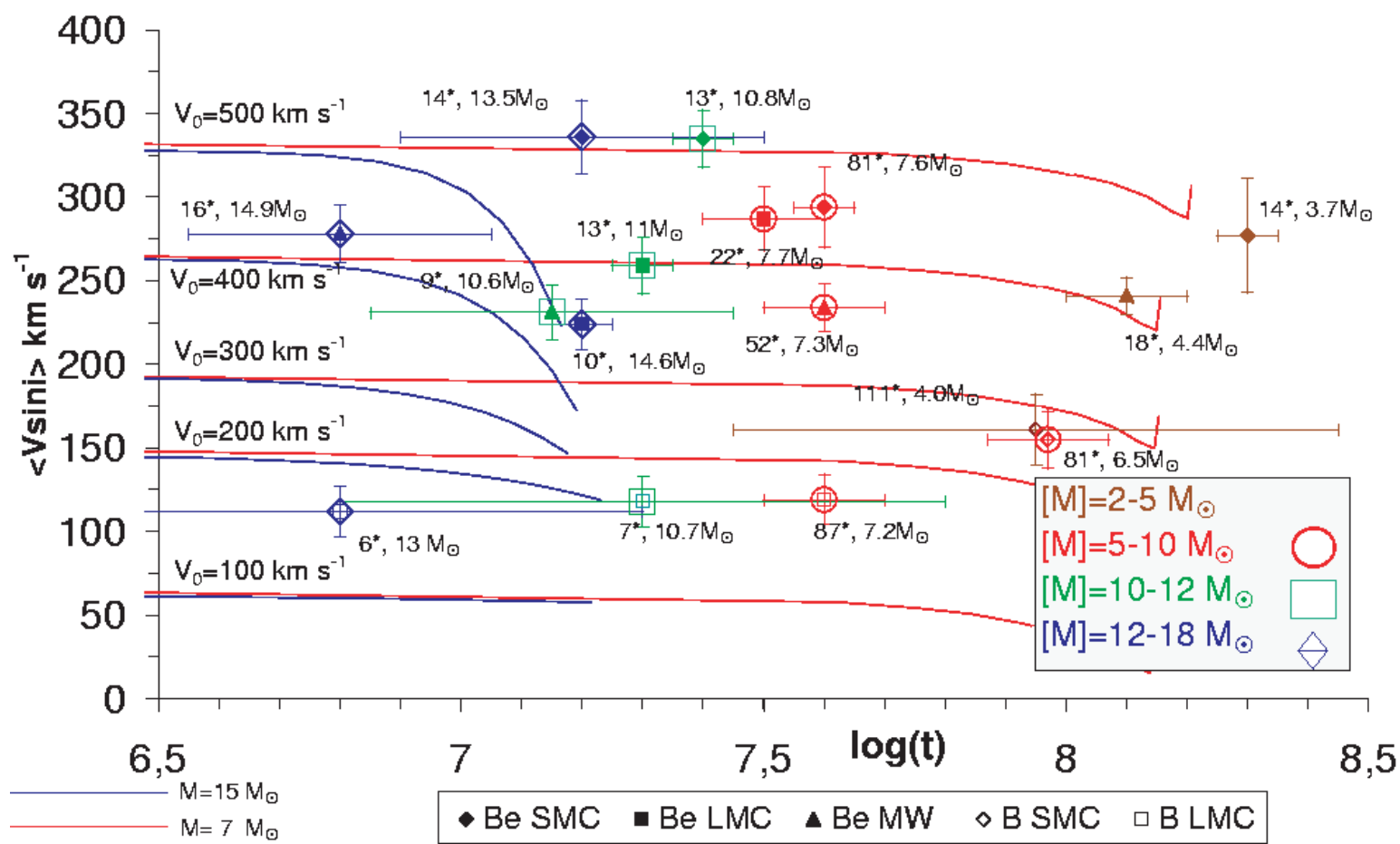

Fig. 8. Comparison of the rotational velocities for Be stars in the SMC, in the LMC, and in the MW; and for B stars in the SMC and the LMC. The squares are for the samples of stars in the LMC, the diamonds are for the samples of stars in the SMC, and the triangles are for the samples of stars in the MW. Empty symbols are for the B stars, and full symbols are for Be stars. The different mass-categories are indicated by different colours and symbols that surround the symbols for the B and Be stars in the 3 galaxies: brown for masses ranging from 2 to $5 M_{\odot}$, red large circles for masses ranging from 5 to $10 M_{\odot}$, green large squares for masses ranging from 10 to $12 M_{\odot}$, and blue large diamonds for masses ranging from 12 to $18 M_{\odot}$. The numbers indicated next to each point correspond to the number of stars "**" in each sample and to their mean mass. The tracks of rotational velocities for a 7 and a $15 M_{\odot}$ star obtained for the SMC from our interpolations in the studies of Meynet \& Maeder (2000, 2002), Maeder \& Meynet (2001) are shown to illustrate the results.

homogeneous possible way, we selected the stars by mass categories: $5 \leq M<10 M_{\odot}, 10 \leq M<12 M_{\odot}$, etc. We assumed a random distribution for the inclination angle.

The number of observed stars for a given mass category is low, therefore we have not separated the stars in clusters and in field categories. This is justified since we have not found any significant difference between the rotational velocities of stars in fields and in clusters. We first present a general result between B and Be stars in the MC, then we study the effects of metallicity and evolution in detail on the rotational velocities for Be stars in the MW and in the MC.

The rotational velocities we derived from observational results for the different samples by mass categories for $\mathrm{B}$ and $\mathrm{Be}$ stars in the MC are reported in Fig. 8. This graph shows, as for spectral type-selection, that $\mathrm{Be}$ stars reach the main sequence with high rotational velocities at the ZAMS in contrast to B stars. Consequently, only a B star with a sufficiently high initial rotational velocity at the ZAMS may become a Be star.

\subsubsection{Effect of metallicity on B stars}

The values of the mean rotational velocities for the masssamples of B stars in the SMC and LMC are given in Table 9 and reported in Fig. 8. For a better comparison, we must reduce the number of degrees of freedom, i.e. compare the samples with similar ages and similar masses and with a sufficient number of elements. That is the case, in this study, for the 5-10 $M_{\odot}$ category sample. Even if the age is not exactly the same, the evolution of the rotational velocity as shown by the tracks for a $7 M_{\odot}$ indicate that the velocity of the SMC's sample is higher than the one of the LMC at exactly the same age. A highly significant difference (probability 99.5\%) in the mean rotational velocity is then shown for this mass-category between the SMC and the LMC with the Student's t-test. Note that such a comparison cannot be made with B stars in the MW, because the data in the literature do not allow any determination of their masses.

The effect of metallicity on rotational velocities for B stars is shown for the 5-10 $M_{\odot}$ category with similar ages between the SMC and the LMC: the lower the metallicity, the higher the rotational velocities of B stars. It could also be valid for other ranges of masses, but this has to be confirmed with appropriate samples. Nevertheless, this observational result nicely confirms the theoretical result of Meynet \& Maeder (2000) and Maeder \& Meynet (2001).

\subsubsection{Effect of metallicity on Be stars}

The selection by mass samples of Be stars in the SMC was made as in Paper I for the LMC. For the MW, we used the studies published by Chauville et al. (2001) and Zorec et al. (2005). The mean rotational velocity for each mass sample of Be stars is reported in Table 10 and is shown in Fig. 8 for the three galaxies. For each sample, the mean age and mass, as well as the number of considered stars, are given.

From the results of the Student's t-test in Table 11, we conclude that there is an effect of metallicity on the rotational velocities for Be stars in samples with similar masses and similar ages: the lower the metallicity, the higher the rotational velocities. This is particularly visible in high-mass $\left(10-12 M_{\odot}\right)$ and 
Table 9. Comparison by mass sub-samples of the mean rotational velocities in the SMC and LMC B stars. For each sub-sample, the mean age, mean mass, mean $V \sin i$ and the number of stars $\left(N^{*}\right)$ are given. No result is given for massive stars in the SMC, because of their small number.

\begin{tabular}{|c|c|c|c|c|c|c|c|c|}
\hline & \multicolumn{4}{|c|}{$\overline{2-5 M_{\odot}}$} & \multicolumn{4}{|c|}{$\overline{5-10 M_{\odot}}$} \\
\hline & $\overline{\langle\text { age }\rangle}$ & $\left\langle M / M_{\odot}\right\rangle$ & $\langle V \sin i\rangle$ & $N^{*}$ & $\langle$ age $\rangle$ & $\overline{\left\langle M / M_{\odot}\right\rangle}$ & $\langle V \sin i\rangle$ & $N^{*}$ \\
\hline SMC B stars & 8.0 & 4.0 & $161 \pm 20$ & 111 & 8.0 & 6.5 & $155 \pm 17$ & 81 \\
\hline LMC B stars & 7.9 & 4.1 & $144 \pm 13$ & 6 & 7.6 & 7.2 & $119 \pm 11$ & 87 \\
\hline & \multicolumn{4}{|c|}{$10-12 M_{\odot}$} & \multicolumn{4}{|c|}{$12-18 M_{\odot}$} \\
\hline & $\overline{\langle\text { age }\rangle}$ & $\left\langle M / M_{\odot}\right\rangle$ & $\langle V \sin i\rangle$ & $N^{*}$ & $\langle\overline{\langle\text { age }\rangle}$ & $\overline{\left\langle M / M_{\odot}\right\rangle}$ & $\langle V \sin i\rangle$ & $N^{*}$ \\
\hline SMC B stars & & & & 3 & & & & 2 \\
\hline LMC B stars & 7.3 & 10.7 & $118 \pm 10$ & 7 & 6.8 & 13.0 & $112 \pm 10$ & 6 \\
\hline
\end{tabular}

Table 10. Comparison by mass sub-samples of the mean rotational velocities for the samples of Be stars in the SMC, LMC and in the MW. For each sample, the mean age, the mean mass, the mean rotational velocity and the number of stars are given. Note no low-mass Be star in the LMC.

\begin{tabular}{lcccccccc}
\hline \hline & \multicolumn{3}{c}{$2-5 M_{\odot}$} & \multicolumn{4}{c}{$5-10 M_{\odot}$} \\
\hline & $\langle$ age $\rangle$ & $\left\langle M / M_{\odot}\right\rangle$ & $\langle V \sin i\rangle$ & $N^{*}$ & $\langle$ age $\rangle$ & $\left\langle M / M_{\odot}\right\rangle$ & $\langle V \sin i\rangle$ & $N^{*}$ \\
\hline SMC Be stars & 8.0 & 3.7 & $277 \pm 34$ & 14 & 7.6 & 7.6 & $297 \pm 25$ & 81 \\
LMC Be stars & & & & 0 & 7.5 & 7.7 & $285 \pm 20$ & 21 \\
MW Be stars & 8.1 & 4.4 & $241 \pm 11$ & 18 & 7.6 & 7.3 & $234 \pm 14$ & 52 \\
\hline & \multicolumn{9}{c}{$10-12$} & $M_{\odot}$ & & & $12-18$ & $M_{\odot}$ & \\
\hline & $\langle$ age $\rangle$ & $\left\langle M / M_{\odot}\right\rangle$ & $\langle V \sin i\rangle$ & $N^{*}$ & $\langle$ age $\rangle$ & $\left\langle M / M_{\odot}\right\rangle$ & $\langle V \sin i\rangle$ & $N^{*}$ \\
\hline SMC Be stars & 7.4 & 10.8 & $335 \pm 20$ & 13 & 7.2 & 13.5 & $336 \pm 40$ & 14 \\
LMC Be stars & 7.3 & 11 & $259 \pm 20$ & 13 & 7.2 & 14.6 & $224 \pm 30$ & 10 \\
MW Be stars & 7.2 & 10.6 & $231 \pm 16$ & 9 & 6.8 & 14.9 & $278 \pm 10$ & 17 \\
\hline
\end{tabular}

intermediate-mass $\left(5-10 M_{\odot}\right)$ samples of Be stars in the SMC and the MW.

Note, moreover, that for the most massive-star samples $(12 \leq$ $\left.M<18 M_{\odot}\right)$ it is more difficult to compare them directly, since the ages are quite different between the MC and MW. We note the lack of massive Be stars in the MW at ages for which Be stars are found in the MC. It suggests that the Be star phase can last longer in low metallicity environments, such as the MC, than in the MW.

\subsection{ZAMS rotational velocities of Be stars}

Interpreting our results requires a set of rotational velocity tracks for masses between 2 and $20 M_{\odot}$, for different metallicities corresponding to the $\mathrm{MC}$ and the $\mathrm{MW}$, and for different initial rotational velocities at the ZAMS. We obtain these tracks by interpolation in the models of the Geneva group as described in Sect. 5.3.1. For the first time we have derived the distributions of the ZAMS rotational velocities of Be stars as shown in Sect. 5.3.2.

\subsubsection{Theoretical evolutionary tracks of the rotational velocity}

We derived rotational velocity evolutionary tracks for different masses, initial velocities and metallicities by interpolation in the curves published by Meynet \& Maeder (2000, 2002), and Maeder \& Meynet (2001). We proceeded as reported in Paper I (Sect. 5.2) for a $7 M_{\odot}$ star. We then obtain curves for 3, 5, 7, 9, 12, 15, and $20 M_{\odot}$ stars, for initial rotational velocities $V_{0}=100,200,300,400,500 \mathrm{~km} \mathrm{~s}^{-1}$, and at available metallicities $Z=0.020$ (solar metallicity), $Z=0.004$ (LMC), and $Z=0.00001$ (metallicity similar to the one of the first generation of stars). For $Z=0.001$ (SMC), the curves result from our interpolations. The increase in the lifetime of stars on the main sequence due to rotation and metallicity is taken into account.
Note that, due to fast internal angular momentum redistribution in the first $\simeq 10^{4}$ years in the ZAMS, the surface rotational velocities decrease by 0.8 to 0.9 times their initial value. Moreover, for the comparison-sake with our observational data, the values plotted are not $V$ but are averaged $V \sin i=(\pi / 4) V$. For example, for an initial rotational velocity equal to $300 \mathrm{~km} \mathrm{~s}^{-1}$, the angular momentum redistribution leads roughly to $V_{\mathrm{ZAMS}}=240 \mathrm{~km} \mathrm{~s}^{-1}$, which corresponds to $V \sin i=(\pi / 4) \times 240 \simeq 190 \mathrm{~km} \mathrm{~s}^{-1}$.

\subsubsection{ZAMS rotational velocities}

Our study shows that Be stars begin their life on the main sequence with higher rotational velocities than those for B stars. For each sample of Be stars in the SMC, LMC, and MW, the initial rotational velocity at the ZAMS has been obtained by interpolation between the tracks of the evolution of rotational velocity during the MS (see Sect. 5.3.1). The resulting distributions of ZAMS rotational velocities for the samples of Be stars in the SMC, LMC, and MW are shown in Fig. 9.

The average rotational velocities at the ZAMS $\left(V_{0}\right)$ are quantified by linear regressions, and their equations are as follows:

- In the SMC, $V_{0}=12.92 \frac{M}{M_{\odot}}+351$ and the correlation coefficient is $R^{2}=0.919$.

- In the MW, $V_{0}=10.91 \frac{M}{M_{\odot}}+277$ and the correlation coefficient is $R^{2}=0.986$.

- For the LMC, the lack of low-mass stars in our sample implies that we cannot determine the ZAMS distribution. The latter seems to be situated between the distributions of the SMC and MW.

\subsubsection{Consequences}

- Whatever the metallicity, the ZAMS rotational velocities of Be stars depend on their masses. 


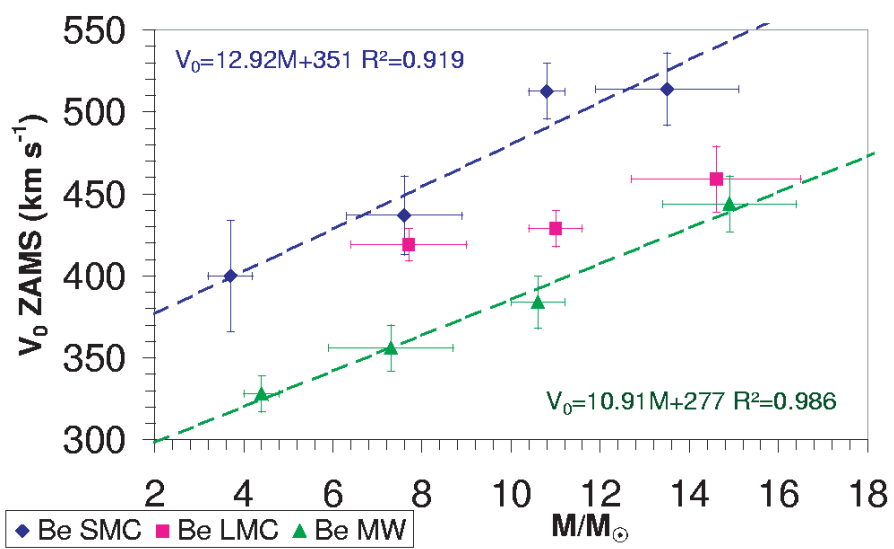

Fig. 9. ZAMS rotational velocities for the samples of Be stars in the SMC (blue diamonds), in the LMC (pink squares), and in the MW (green triangles). The dashed lines correspond to the linear regressions. Their corresponding equations and correlation coefficient are given in the upper left and lower right corners.

- Following the SMC and MW curves, the trend (the gradient) of the ZAMS rotational velocities of Be stars could be independent of the metallicity.

- There is an effect of metallicity on the distributions of the ZAMS rotational velocities. The lower the metallicity, the higher the ZAMS rotational velocities.

- There is a limit for the ZAMS rotational velocities below which B stars will never become Be stars. This limit depends on the metallicity.

The initial conditions (magnetic field, accretion disk, etc.) in an open cluster will lead to a more or less high number of B stars with a ZAMS rotational velocity high enough to become Be stars. Therefore, the rates of Be stars will fluctuate depending on the cluster. It is expected that the mean rate of Be stars at low metallicity, typically in the SMC, is higher than at high metallicity, typically in the MW. This trend has already been observed by Maeder et al. (1999) in open clusters. We find a similar result between the fields in the LMC and the SMC (Martayan et al. 2006 c, in preparation). All these observational results place new constraints on the pre-main sequence (PMS) evolution of B and Be stars progenitors and, in particular, support the arguments of Stepién (2002) about the influence of a magnetic field on the formation conditions of B and Be stars (see Paper I, Sect. 5.2.5). Progenitors of Be stars should possess a weak magnetic field with a surface intensity between 40 and $400 \mathrm{G}$ and, due to the short PMS phase for the early types, would conserve their strong rotational velocity during the main sequence. In low metallicity environments, the magnetic field has less of a braking impact, which could explain why Be stars in the SMC can rotate initially with higher velocities than in the LMC, and why Be stars in the LMC with higher velocities than in the MW, as shown in Figs. 7 and 8. Note that a weak magnetic field is suspected in the classical Be star $\omega$ Ori (Neiner et al. 2003).

\section{Angular velocity and metallicity: results and discussion}

Thanks to the formulae published in Chauville et al. (2001) and in Paper I, it is possible to obtain the average ratio of angular to the breakup angular velocity for the stars.

\subsection{Angular velocities for $B$ stars}

We have determined the mean $\Omega / \Omega_{\mathrm{c}}$ ratio of B-type stars in the LMC $(37 \%)$ and in the SMC $(58 \%)$. In the same way, we determine this ratio in the MW with the values in Table 7; and it ranges from 30 to $40 \%$. The values of $\Omega / \Omega_{\mathrm{c}}$ seem to be similar for B stars in the MW and in the LMC, but higher in the SMC. This difference is probably due to the large difference in metallicity between the SMC and the LMC/MW. We recall that the considered stars in the MC have similar ages.

\subsection{Angular velocities for Be stars}

According to Porter (1996), the mean $\Omega / \Omega_{\mathrm{c}}$ ratio of Be-type stars in the MW is $84 \%$ agrees well with the one $(83 \%)$ determined by Chauville et al. (2001). Following the detailed study by Cranmer (2005) of Be stars in the MW, the ratio $\Omega / \Omega_{\mathrm{c}}$ ranges from $69 \%$ to $96 \%$ for the early-types. In the LMC (Paper I) this ratio ranges from $73 \%$ to $85 \%$, and in the SMC (this study) from $94 \%$ to $100 \%$. We recall that, in the MC, the observed Be stars are also early-types and have similar ages. We thus note the following trend: in the SMC they rotate faster than in the LMC/MW and are close to the breakup velocity or are critical rotators. This shows that, in a low metallicity environment such as the SMC, more massive Be stars can reach the critical velocity. We also note that Be stars appear with at least $\Omega / \Omega_{\mathrm{c}} \simeq 70 \%$ in the LMC. This value seems to be a threshold value for obtaining a Be star in the MW, LMC, and by extension certainly in the SMC.

According to the stellar wind theories and Maeder \& Meynet (2001), the higher the mass of the star, the higher the mass loss and angular momentum loss. However, in low-metallicity environments, this mass loss and, consequently, the angular momentum loss are lower than in the MW. As the radius of the star increases during the main sequence and as the star conserves high rotational velocities with a mass that decreases only slightly, the critical velocity decreases. Consequently, the $\Omega / \Omega_{\mathrm{c}}$ ratio for massive stars increases at low metallicity, while it decreases in the MW. For intermediate and low mass Be stars, whatever the metallicity, the $\Omega / \Omega_{\mathrm{c}}$ ratio first stays relatively constant and then increases at the end of the main sequence.

\subsection{ZAMS angular velocities for Be stars}

Thanks to both distributions of ZAMS rotational velocities for Be stars presented in Sect. 5.3 and to the mass, and radius at the ZAMS from the Geneva models, it is possible to obtain the ZAMS angular velocities and the $\Omega / \Omega_{\mathrm{c}}$ ratio for Be stars in the MW and in the MC. The results are given in Table 12. The effect of metallicity on the angular velocities is visible at the ZAMS. Note that the ratio $\Omega / \Omega_{\mathrm{c}}$ increases as the mass of the star increases.

All the theoretical calculations from Meynet \& Maeder (2000, 2002) and Maeder \& Meynet (2001) were performed with a ZAMS rotational velocity equal to $300 \mathrm{~km} \mathrm{~s}^{-1}$. From the observations, we find that Be stars begin their MS lifetime with ZAMS rotational velocities higher than $300 \mathrm{~km} \mathrm{~s}^{-1}$. For example, for a $20 M_{\odot}$ Be star, the ZAMS rotational velocity is equal to $495 \mathrm{~km} \mathrm{~s}^{-1}$ in the MW and $609 \mathrm{~km} \mathrm{~s}^{-1}$ in the SMC. The ZAMS rotation rate for a B star in the SMC is thus higher than the value adopted by Meynet \& Maeder in their studies. In the SMC, it is consequently easier than expected for Be stars to reach the critical velocity. Note that, due to differential rotation, these stars could be critical rotators at their surface but not inside their core. 
Table 12. Ratio of angular velocity to the breakup angular velocity $\Omega / \Omega_{\mathrm{c}}(\%)$ at the ZAMS for Be stars in the MW and in the SMC (this study) for a $5,12,20 M_{\odot}$ star.

\begin{tabular}{ccc}
\hline \hline & MW & SMC \\
\hline $5 M_{\odot} \Omega / \Omega_{\mathrm{c}}(\%)$ & 75 & 77 \\
$12 M_{\odot} \Omega / \Omega_{\mathrm{c}}(\%)$ & 80 & 84 \\
$20 M_{\odot} \Omega / \Omega_{\mathrm{c}}(\%)$ & 85 & 92 \\
\hline
\end{tabular}

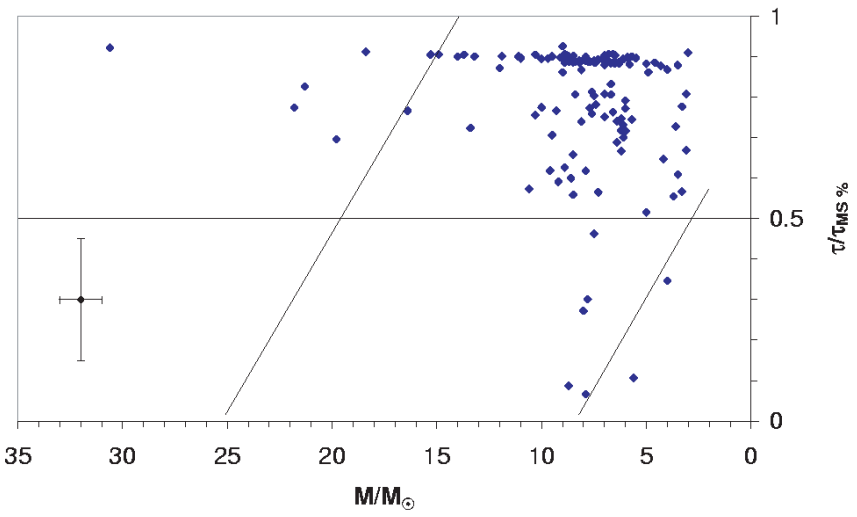

Fig. 10. Evolutionary status of Be stars in the SMC. The fast rotation effects are taken into account with $\Omega / \Omega_{\mathrm{c}}=95 \%$. The typical errors are shown in the lower left corner. The diagonals show the area of existing Be stars in the MW (Zorec et al. 2005).

\section{Evolutionary status of Be stars}

To investigate the evolutionary status of Be stars in the SMC taking into account the effects of fast rotation and the low metallicity $(Z=0.001)$ of the SMC, we first calculate the lifetime of the main sequence $\left(\tau_{\mathrm{MS}}\right)$ of massive stars by interpolation in grids of the evolutionary tracks provided by Maeder \& Meynet (2001) and Meynet \& Maeder (2000) for different metallicities, with an initial velocity $V_{0}=300 \mathrm{~km} \mathrm{~s}^{-1}$ and for stars with masses higher than $9 M_{\odot}$. Second, we extrapolated the $\tau_{\text {MS }}$ values towards lower masses (5-9 $M_{\odot}$ ). We then investigated the evolutionary status $\frac{\tau}{\tau_{\mathrm{MS}}}$ of the SMC Be stars using the values of their ages corrected from fast rotation effects and given in Table 6 . Results are shown in Fig. 10.

The following remarks can be made:

- It appears that more massive Be stars in the SMC are evolved, since all of them in our sample are localized in the second part of the MS.

- Intermediate mass Be stars are scattered across the MS.

- Less massive Be stars are mainly evolved and in the second part of the MS $\left(\frac{\tau}{\tau_{\mathrm{MS}}} \geq 0.5\right)$.

To compare the evolutionary status of Be stars in the different galaxies, we calculated the $\frac{\tau}{\tau_{\mathrm{MS}}}$ ratio for massive and less massive stars in the LMC $(Z=0.004)$ and the MW (solar metallicity) as for the SMC. We note that in the MW, our results are quite similar to the ones in Zorec et al. (2005). However, in the LMC, our previous results in Paper I (Fig. 12) are slightly modified with the use of evolutionary tracks adapted to its metallicity $(Z=0.004)$, mainly for the less massive Be stars that appear more evolved in the present study. The proportions of more massive $\left(>12 M_{\odot}\right)$ and less massive $\left(\leq 12 M_{\odot}\right)$ Be stars in the SMC,
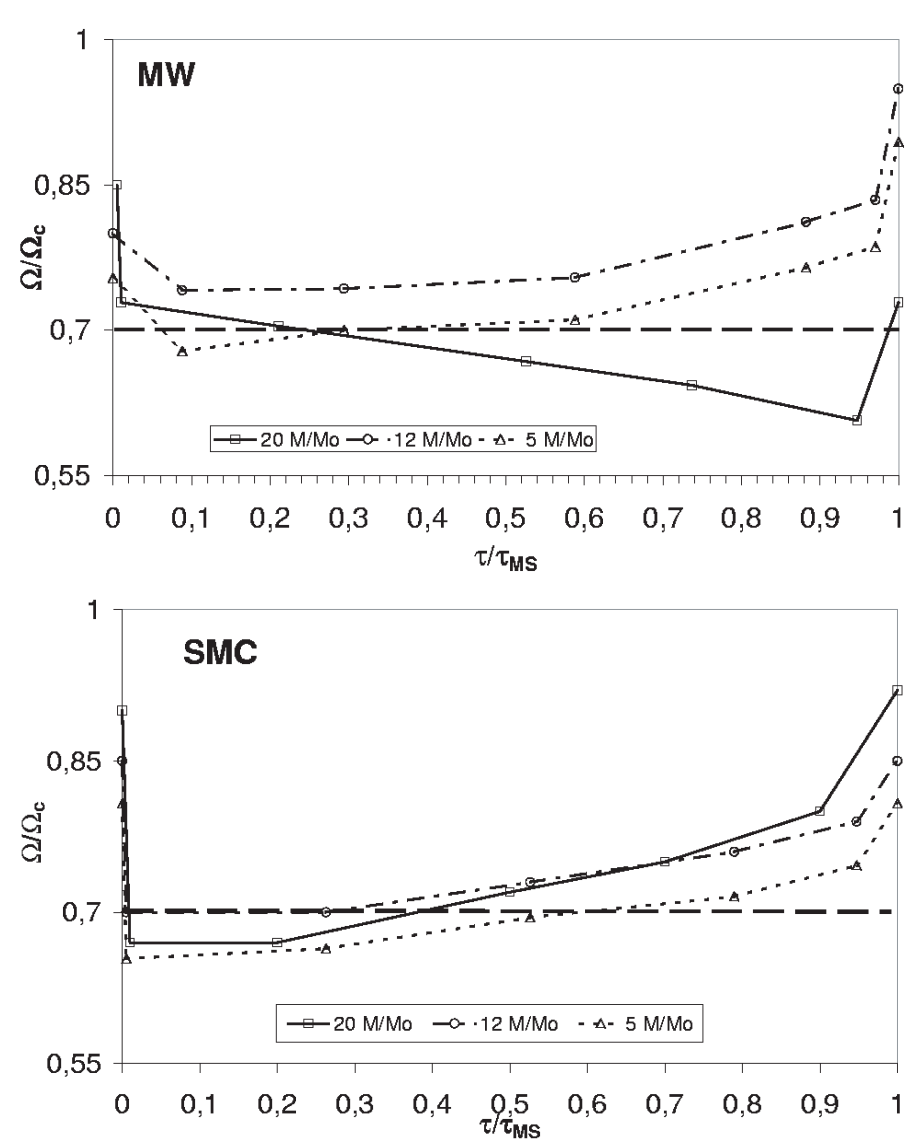

Fig. 11. Evolution of the $\Omega / \Omega_{\mathrm{c}}$ ratio for 3 types of stars: $20 M_{\odot}$ (squares), $12 M_{\odot}$ (circles) and $5 M_{\odot}$ (triangles) stars in the MW (top) and in the SMC (bottom). The stars may become Be stars if $\Omega / \Omega_{\mathrm{c}} \geq$ $70 \%$ (noted with a long dashed line).

Table 13. Proportions in SMC, LMC, and MW of Be stars (in \%) in the upper $\left(\frac{\tau}{\tau_{\mathrm{MS}}}>0.5\right)$ and lower $\left(\frac{\tau}{\tau_{\mathrm{MS}}} \leq 0.5\right) \mathrm{MS}$ for masses $>12 M_{\odot}$ and $\leq 12 M_{\odot} .{ }^{*}$ values come from from Zorec et al. (2005).

\begin{tabular}{cccc}
\hline \hline \multicolumn{5}{c}{$M>12 M_{\odot}$} \\
\hline$\frac{\tau}{\tau_{\mathrm{MS}}}>0.5$ & 30 & 100 & 100 \\
$\frac{\tau}{\tau_{\mathrm{MS}}} \leq 0.5$ & 70 & 0 & 0 \\
\hline \multicolumn{5}{c}{$M \leq 12 M_{\odot}$} \\
\hline$\frac{\tau}{\tau_{\mathrm{MS}}}>0.5$ & 65 & LMC & SMC \\
$\frac{\tau}{\tau_{\mathrm{MS}}} \leq 0.5$ & 35 & 23 & 64 \\
\hline
\end{tabular}

LMC, and MW are summarised in Table 13. It is shown that all the more massive stars in the LMC and the SMC are in the upper part of the MS $\left(\frac{\tau}{\tau_{\mathrm{MS}}} \geq 0.5\right)$, in contrast to the MW where they are mainly in the lower part of the MS. The less massive stars seem to follow the same trend in the MW and the MC, and they are mainly in the upper part of the MS in agreement with Fabregat \& Torrejon (2000). With our results from the ZAMS rotational velocity distributions (Fig. 9) and with the theoretical evolution of angular velocities taken from the Geneva models 
mentioned above, we obtained the evolution of the angular velocities for different masses $\left(5,12,20 M_{\odot}\right)$ of Be stars in the MW and the SMC as shown in Fig. 11. We have emphasised the significant behaviour differences between Be stars as a function of their mass and of their metallicity environment, and we propose the following explanation of the Be phenomenon in the MW and in the MC:

- More massive Be stars: In the MW, more massive stars begin their lives in the MS with a high $\Omega / \Omega_{\mathrm{c}}(>70 \%$, see Fig. 9 and Table 12), thus these stars could be Be stars. Then, by angular momentum loss, the stars spin down and might not eject matter anymore; thus, they lose their "Be star" character during the first part of the MS (typically for $\frac{\tau}{\tau_{\mathrm{MS}}}>0.2$ ). However, at the end of the MS $\left(\frac{\tau}{\tau_{\mathrm{MS}}} \simeq 1\right)$, during the secondary contraction, massive stars could have $\Omega / \Omega_{\mathrm{c}}$ high enough to become Be stars again.

In the MC, more massive stars begin their lives in the MS with a high $\Omega / \Omega_{\mathrm{c}}(>70 \%)$ and could be Be stars, but only at the very beginning of the ZAMS. Therefore, they rapidly lose "the Be star status". However, they spin up (increase of the radius, low mass-loss, and low angular-momentum loss) and reach very high $\Omega / \Omega_{\mathrm{c}}$ at the end of the first part of the MS (typically after $\frac{\tau}{\tau_{\mathrm{MS}}}>0.4$ ), so that they become Be stars again, contrary to what is observed in the MW.

- Intermediate-mass Be stars: In the MW as in the MC, intermediate mass Be stars begin their life in the MS with high $\Omega / \Omega_{\mathrm{c}}(>70 \%$, Fig. 9 and Table 12$)$, therefore the stars could be Be stars. Then, the evolution of their $\Omega / \Omega_{\mathrm{c}}$ allows these stars to remain Be stars.

- Less massive Be stars: In the MW as in the MC, less massive stars begin their lives on the MS with $\Omega / \Omega_{\mathrm{c}}$ high enough to obtain the status of "Be stars". After the fast internal angular redistribution in the first $\simeq 10^{4}$ years in the ZAMS, the surface rotational velocities decrease and the stars lose the status of "Be stars". Then, the evolution of their $\Omega / \Omega_{\mathrm{c}}$ allows these stars to become Be stars again (typically after $\frac{\tau}{\tau_{\mathrm{MS}}}>0.5$ ), in agreement with Fabregat \& Torrejón (2000).

We note that our propositions explain results presented by Zorec et al. (2005, their Fig. 6) and our results concerning the LMC (Paper I) and the SMC (Fig. 10). We also notice no difference between the evolutionary status of Be stars in clusters and fields. In summary, a B star can become a Be star, if its progenitor has a strong initial rotational velocity at the ZAMS. Depending on the metallicity of the environment of a star and on the stellar mass, the Be phenomenon appears at different stages of the MS.

\section{Conclusions}

With the VLT-GIRAFFE spectrograph, we obtained spectra of a large sample of B and Be stars in the SMC-NGC 330 and surrounding fields. We determined fundamental parameters for B stars in the sample and apparent and parent non-rotating counterpart (pnrc) fundamental parameters for Be stars.

Using results from this study and those obtained for the LMC with the same instrumentation (Paper I), we made a statistical comparison of the behaviour of B and Be stars in the Magellanic Clouds with the MW.

- There is no difference in rotational velocities between earlytype stars in clusters and in fields in the LMC and in the SMC.
- The lower the metallicity is, the higher the rotational velocities are. The B and Be stars rotate faster in the SMC than in the LMC and faster in the LMC than in the MW.

- We determined, for the first time, the distributions of the ZAMS rotational velocities of Be stars. The ZAMS rotational velocities are mass-dependent and metallicitydependent. The gradients of the distributions seem to be similar whatever the metallicity.

- Only a fraction of the B stars that reach the ZAMS with sufficiently high initial rotational velocities can become Be stars. Wisniewski \& Bjorkman (2006) seem to have found a similar result photometrically.

- The angular velocities are similar for B stars in the LMC and in the MW and lower than those in the SMC. The same result was obtained for Be stars.

- More massive Be stars in the SMC, which are evolved, are critical rotators.

- In an evolutionary scheme, massive Be stars in the MC and more particularly in the SMC appear in the second part of the main sequence, in contrast to massive Be stars in the MW, which appear in the first part of the main sequence.

- Other categories of Be stars (intermediate and less massive Be stars) follow the same evolution whatever the metallicity of the environment.

Our results support Stepién's scenario (2002): massive stars with a weak or moderate magnetic field and with an accretion disk during at least $10 \%$ of their PMS lifetime would reach the ZAMS with sufficiently high initial rotational velocity to become Be stars. Our observational results also support theoretical results by Meynet \& Maeder $(2000,2002)$ and Maeder \& Meynet (2001) for massive stars. In a more general way, they illustrate the behaviour of massive stars and the importance of the processes linked to rotation and to metallicity. In particular, our results show that rotation plays a major role in the behaviour of massive stars. Moreover, this pioneer study of a large sample of B stars in low-metallicity environments, such as the LMC (Paper I) and the SMC (this study), allows us to approach the "first stars". We can reasonably expect that first massive stars are fast rotators.

In forthcoming papers, we will present results of $\mathrm{CNO}$ abundance determinations, discuss the proportion of Be stars, and report on the discovery of binaries in the SMC.

Acknowledgements. We would like to thank Dr V. Hill for performing the observing run in September 2004 with success and good quality. We thank Drs C. Ledoux, P. François, and E. Depagne for their help during the observing run in October 2003. This research has made use of the Simbad database and Vizier database maintained at the CDS, Strasbourg, France, as well as of the WEBDA database. Y.F. acknowledges financial support from the Belgian Federal Science Policy (projects IAP P5/36 and MO/33/018).

\section{References}

Castelli, F., Gratton, R. G., \& Kurucz, R. L. 1997, A\&A, 318, 841 Chauville, J., Zorec, J., Ballereau, D., et al. 2001, A\&A, 378, 861 Chiosi, E., Vallenari, A., Held, E. V., et al. 2006, A\&A, 452, 179 Cranmer, S. R. 2005, ApJ, 634, 585

Fabregat, \& J., Torrejón, J. M. 2000, A\&A, 357, 451

Frémat, Y., Zorec, J., Hubert, A.-M., et al. 2005, A\&A, 440, 305

Frémat, Y., Neiner, C., Hubert, A.-M., et al. 2006, A\&A, 451, 1053

Glebocki, \& R., Stawikowski, A. 2000, Acta Astr., 50, 509

Grebel, E. K., Richtler, T., \& de Boer, K. S. 1992, A\&A, 254, L5

Hubeny, I., \& Lanz, T. 1995, ApJ, 439, 875

Jasniewicz, G., \& Thévenin, F. 1994, A\&A, 282, 717 
Keller, S. C., Wood, P. R. \& Bessell, M. S. 1999, A\&AS, 134, 489

Keller, S. C. 2004, PASA, 21, 310

Kurucz, R. L. 1993, Kurucz CE-ROM No. 13 (Cambridge, Mass.: Smithsonian Astrophysical Observatory)

Levato, H. \& Grosso, M. 2004, IAUS, 215, 51

Martayan, C., Hubert, A.-M., Floquet, M., et al. 2005a, Active OB stars meeting, Sapporo Japan, 28/08-02/09/2005, ed. S. Stefl, S. Owocki, A. Okazaki, ASP Conf. Ser. [arXiv: astroph0602149]

Martayan, C., Floquet, M., Hubert, A.-M., et al. 2005b, Active OB stars meeting, Sapporo Japan, 28/08-02/09/2005, ed. S. Stefl, S. Owocki, A. Okazaki ASP Conf. Ser. [arXiv: astroph0602148]

Martayan, C., Hubert, A.-M., Floquet, M., et al. 2006a, A\&A, 445, 931 (M06)

Martayan, C., Frémat, Y., Hubert, A.-M., et al. 2006b, A\&A, 452, 273 (Paper I)

Martayan, C., Floquet, M., Hubert, A.-M., et al. 2006c, A\&A, in preparation

Maeder, A., \& Meynet, G. 2001, A\&A, 373, 555
Maeder, A., Grebel, E. K., \& Mermilliod, J.-C. 1999, A\&A, 346, 459 Meynet, G., \& Maeder, A. 2000, A\&A, 361, 101

Meynet, G., \& Maeder, A. 2002, A\&A, 390, 561

Momany, Y., Vandame, B., Zaggia, S., et al. 2001, A\&A, 379, 436

Neiner, C., Hubert, A.-M., Frémat, Y., et al. 2003, A\&A, 409, 275

Pietrzyński, G., \& Udalski, A. 1999, Acta Astr., 49, 157

Porter, J. M. 1996, MNRAS, 280, 31

Rafelski, M., \& Zaritsky, D. 2005, AJ, 129, 2701

Schaller, G., Schaerer, D., Meynet, G., \& Maeder, A. 1992, A\&AS, 96, 269

Stepień, K. 2002, A\&A, 383, 218

Townsend, R. H. D., Owocki, S. P., \& Howarth, I. D. 2004, MNRAS, 350, 189

Wisniewski, J. P., \& Bjorkman, K. S. 2006, [arXiv: astroph0606525]

Yudin, R. V. 2001, A\&A, 368, 912

Zorec, J., Frémat, \& Y., Cidale, L. 2005, A\&A, 441, 235 
C. Martayan et al.: B and Be stars in the SMC. II., Online Material $p 1$

\section{Online Material}


Table 1. Fundamental parameters for O, B, A stars in the SMC. The name, coordinates $(\alpha(2000), \delta(2000)), V$ magnitude and $(B-V)$ colour index of stars are taken from the EIS catalogues. The effective temperature $T_{\text {eff }}$ is given in $\mathrm{K}, \log g$ in dex, $V \sin i$ in $\mathrm{km} \mathrm{s}^{-1}$ and $R V$ in km s $\mathrm{k}^{-1}$. The $1 \sigma$ error is given for each parameter. The abbreviation "CFP" is the spectral type and luminosity classification determined from fundamental parameters (method 2), whereas "CEW" is the spectral type and luminosity classification determined from EW diagrams (method 1). The localization in clusters is indicated in the last column: cl0 for NGC $330\left(0 \mathrm{~h} 56 \min 19 \mathrm{~s}-72^{\circ} 27^{\prime} 52^{\prime \prime}\right)$, cl1 for H $86170\left(0 \mathrm{~h} 56 \min 21 \mathrm{~s}-72^{\circ} 21^{\prime} 12^{\prime \prime}\right)$, cl2 for [BS95]78 $\left(0 \mathrm{~h} 56 \min 04 \mathrm{~s}-72^{\circ} 20^{\prime} 12^{\prime \prime}\right)$, cl3 for the association SMC ASS 39 (0h56min6s $\left.-72^{\circ} 18^{\prime} 00^{\prime \prime}\right)$, cl4 for OGLE-SMC109 $\left(0 \mathrm{~h} 57 \mathrm{~min} 29.8 \mathrm{~s}-72^{\circ} 15^{\prime} 51.9^{\prime \prime}\right)$, cl5 for NGC299 (0h53min24.5s $\left.-72^{\circ} 11^{\prime} 49^{\prime \prime}\right)$ corrected coordinates, cl6 for NGC306 (0h54min15s $\left.-72^{\circ} 14^{\prime} 30^{\prime \prime}\right)$, cl7 for H86 145 (0h53min37s $\left.-72^{\circ} 21^{\prime} 00^{\prime \prime}\right)$, cl8 for OGLE-SMC99 $\left(0 \mathrm{~h} 54 \min 48.24 \mathrm{~s}-72^{\circ} 27^{\prime} 57.8^{\prime \prime}\right)$.

\begin{tabular}{|c|c|c|c|c|c|c|c|c|c|c|c|c|}
\hline$\overline{\text { Star }}$ & $\overline{\alpha(2000)}$ & $\overline{\delta(2000)}$ & $\bar{V}$ & $\overline{B-V}$ & $\overline{S / N}$ & $\overline{T_{\text {eff }}}$ & $\log g$ & $V \sin i$ & $\overline{R R V}$ & $\overline{\mathrm{CFP}}$ & $\overline{\text { CEW }}$ & $\mathrm{co}$ \\
\hline SMC5_000351 & 05332.810 & -722643.70 & 17.75 & -0.15 & 20 & $16500 \pm 1600$ & $4.4 \pm 0.2$ & $312 \pm 47$ & $158 \pm 10$ & $\bar{B} 3 \mathrm{~V}$ & B2.5IV & \\
\hline SMC & 0 & 2254 & 4.27 & -0.12 & 90 & $00 \pm 400$ & $2.7 \pm 0.1$ & 104 & $138 \pm 10$ & B5II-III & $0.5 \mathrm{III}$ & \\
\hline & & 22 & 1( & -0.14 & 60 & 00 & .2 & 16 & 10 & B3IV & B2III & \\
\hline & 5 & 72210 & .97 & -0.20 & 60 & $20000 \pm$ & $4.2 \pm 0.2$ & & 0 & $\mathrm{~B} 2 \mathrm{~V}$ & B2IV & \\
\hline 660 & 29 & 722023.36 & .18 & -0.24 & 60 & 00 & $4.2 \pm 0.2$ & $201 \pm 16$ & $49 \pm 10$ & B3V & B2.5III & \\
\hline 670 & 1 & -722014.84 & .10 & -0.17 & 65 & $0 \pm 900$ & $3.9 \pm 0.2$ & $52 \pm 10$ & $9 \pm 10$ & B2IV & B2IV & \\
\hline 810 & 561 & -721728.40 & .41 & -0.21 & 65 & $33000 \pm 1700$ & $4.0 \pm 0.2$ & $163 \pm 18$ & $134 \pm 10$ & O9V & O9.5III & \\
\hline 889 & .000 & -721624.20 & 7.46 & -0.00 & 30 & 200 & $3.7 \pm 0.2$ & $140 \pm 21$ & $162 \pm 10$ & B8IV & 36.5III & \\
\hline 5_000924 & 635 & -721546.25 & 17.59 & -0.02 & 50 & \pm 1200 & $4.4 \pm 0.2$ & $291 \pm 28$ & $168 \pm 10$ & $\mathrm{~B} 2 \mathrm{~V}$ & B2IV & \\
\hline _000959 & 5600.520 & -721500.80 & 17.23 & -0.21 & 35 & $500 \pm 1800$ & $4.0 \pm 0.2$ & $170 \pm 25$ & $151 \pm 10$ & $\mathrm{~B} 2 \mathrm{~V}$ & B2III & \\
\hline 2782 & 5441.610 & -722815.70 & 16.09 & -0.24 & 40 & $25000 \pm 1700$ & $4.1 \pm 0.2$ & $81 \pm 10$ & $170 \pm 10$ & B1V & B0V & $\mathrm{cl} 8$ \\
\hline C5_003118 & 605.580 & -722621.40 & 5.54 & -0.15 & 50 & $20000 \pm 1300$ & $3.8 \pm 0.2$ & $159 \pm 13$ & $85 \pm 10$ & B2IV & $31 \mathrm{III}$ & \\
\hline 3175 & 42.450 & -722553.60 & 5.96 & -0.18 & 45 & $2000 \pm 1700$ & $4.0 \pm 0.2$ & $84 \pm 10$ & $81 \pm 10$ & B2V & B1IV & \\
\hline 195 & 57.050 & -722545.67 & 17.66 & -0.18 & 45 & $17500 \pm 1300$ & $4.5 \pm 0.2$ & $69 \pm$ & $117 \pm 10$ & B3V & B3IV & \\
\hline 292 & 3 & -722513.75 & 5.89 & -0.17 & 65 & 100 & $4.0 \pm 0.2$ & 114 & $135 \pm 10$ & $\mathrm{~B} 2 \mathrm{~V}$ & B2IV & \\
\hline & 0 & .49 & 15 & -0.23 & 40 & 00 & 0.2 & 6 & 10 & B1V & $31 \mathrm{~V}$ & \\
\hline & & .00 & 4 & -0.17 & 20 & 17( & 0.2 & 4 & 0 & B3V & 3III & \\
\hline 39 & 0 & 722229.90 & 9 & -0.11 & 120 & 0 & 0.1 & 10 & $160 \pm 10$ & $11-1$ & O9III & \\
\hline 09 & 0 & 722200.50 & .90 & -0.11 & 25 & 00 & 0.2 & 202 & 0 & $V$ & 3III & \\
\hline 55 & 0 & 722153.90 & 45 & -0.25 & 120 & 0 & 0.1 & 10 & 10 & $01 \mathrm{~V}$ & $7 \mathrm{~V}$ & \\
\hline 910 & 0 & -722133.30 & 4.86 & -0.16 & 55 & 00 & 0.2 & 10 & 10 & B2III & $\mathrm{O} 8 \mathrm{~V}$ & cl1 \\
\hline 942 & 6 & -722121.58 & 7.27 & -0.22 & 50 & 00 & 0.2 & 15 & 10 & $\mathrm{~B} 2 \mathrm{~V}$ & B1.5IV & \\
\hline 998 & 5603.277 & -722101.78 & 7.70 & -0.21 & 40 & 00 & 0.2 & 19 & 10 & $5 I V$ & B3III & \\
\hline 999 & 05629.530 & -722101.90 & 16.52 & -0.20 & 40 & $1 / 000 \pm$ & $3.5 \pm 0.2$ & $87 \pm 10$ & $51 \pm 10$ & B3IV & $1.5 \mathrm{III}$ & \\
\hline 25 & 5548.274 & 722052.50 & 7.83 & -0.16 & 40 & 00 & $4.3 \pm 0.2$ & $153 \pm 15$ & $3 \pm 10$ & B3V & 3III & \\
\hline & 570 & -722050.80 & 5.43 & -0.23 & 45 & 00 & $3.5 \pm 0.2$ & 10 & & B2IV & $0.5 \mathrm{III}$ & \\
\hline & & -122 & 7.26 & -0.23 & 60 & & 0.2 & & $43 \pm 10$ & $B 21 V-V$ & 2III & \\
\hline & 0 & -722024.60 & 5.12 & -0.02 & 45 & & 0.2 & 36 & 10 & 1III & 37III & \\
\hline & & 722 & .39 & -0.14 & 55 & 000 & 0.2 & & & $3 \mathrm{IV}$ & B1.5III & \\
\hline & & 722 & 38 & -0.10 & 40 & & 0.2 & & 10 & III & 3 IIII & 12 \\
\hline & & 722 & 3 & -0.19 & 40 & 00 & 0.2 & & 10 & $3 \mathrm{IV}$ & 2III & \\
\hline & & -722 & 7.03 & -0.18 & 70 & & 0.2 & & 10 & IV & 3III & \\
\hline & & -7220 & .13 & -0.28 & 70 & 0 & 0.2 & & 10 & $\mathrm{~B} 2 \mathrm{~V}$ & B2III & \\
\hline & 0 & -72200 & .29 & -0.20 & 30 & 00 & $4.2 \pm 0.2$ & 28 & 10 & $\mathrm{~V}$ & B2III & \\
\hline 98 & 00 & -721953.80 & 14.97 & -0.19 & 60 & 00 & $3.8 \pm 0.2$ & 10 & 10 & O9IV & $\mathrm{O} 4.5 \mathrm{~V}$ & \\
\hline 203 & 340 & -721952.90 & 17.54 & -0.28 & 20 & 00 & $4.3 \pm 0.2$ & 29 & $120 \pm 10$ & $\mathrm{~B} 2 \mathrm{~V}$ & B2III & \\
\hline 263 & 1 & -721932.85 & 17.43 & -0.15 & 45 & 00 & 0.2 & 32 & \pm 10 & $33 I V$ & 3 III & \\
\hline 326 & 51.738 & -721910.54 & 17.05 & -0.15 & 55 & 0 & $4.1 \pm 0.2$ & 30 & \pm 10 & B1V & O9V & \\
\hline 81 & 18.570 & -721855.00 & 17.99 & -0.25 & 20 & 000 & $4.4 \pm 0.2$ & $29 \pm 10$ & $71 \pm 10$ & $\mathrm{~B} 2 \mathrm{~V}$ & $32 \mathrm{IV}$ & \\
\hline 13 & 0 & -721845.00 & 16.10 & -0.17 & 35 & 00 & $3.2 \pm 0.2$ & 25 & $121 \pm 10$ & B7III & B2.5III & \\
\hline & & -721 & 17.71 & -0.23 & 50 & 00 & 0.2 & & 15 & B3V & B3V & \\
\hline 02 & 10 & -721 & 16.28 & -0.26 & 35 & & 0.2 & 22 & & IIV & $9.5 \mathrm{III}$ & \\
\hline & 50 & -721 & 16.04 & -0.25 & 45 & 00 & 0.2 & & & B2IV & B1.5III & \\
\hline & & -72 & 16.38 & -0.09 & 40 & & & & & B3III & 2III & \\
\hline & 0 & -721 & 17.89 & -0.14 & 20 & & & & & & 2III & \\
\hline & 0 & -7217 & 15.37 & -0.21 & 70 & & 0.2 & & & B0V & O9IV & \\
\hline & 0 & -721706.00 & 16.13 & -0.21 & 50 & & 0.2 & & 10 & 32III-IV & 1III & \\
\hline & & -7216 & 17.83 & -0.20 & 20 & & 0.2 & & & $\mathrm{~B} 2 \mathrm{~V}$ & $2 \mathrm{IV}$ & \\
\hline & 13 & -721607.40 & 17.81 & -0.15 & 25 & & $4.4 \pm 0.2$ & 167 & $132 \pm 10$ & B3V & 3 IIII & \\
\hline & 30 & -721602.20 & 17.91 & -0.22 & 30 & & $4.2 \pm 0.2$ & $221 \pm 33$ & $167 \pm 10$ & B2V & B2III & S1 \\
\hline & & -721 & 17.90 & -0.18 & 25 & & $4.3 \pm 0.2$ & & $136=$ & B3V & B3III & \\
\hline SMC5_004988 & 005649.690 & -721529.60 & 15.72 & -0.17 & 55 & $20000 \pm$ & $3.7 \pm 0.2$ & $134 \pm 10$ & $164 \pm$ & B2IV & B1III & \\
\hline
\end{tabular}


Table 1. continued.

\begin{tabular}{|c|c|c|c|c|c|c|c|c|c|c|c|c|}
\hline$\overline{\text { Star }}$ & $\overline{\alpha \alpha(2000)}$ & $\overline{\overline{\delta(2000)}}$ & $\overline{\bar{V}}$ & $\overline{B-V}$ & $\overline{S S / N}$ & $\overline{\overline{T_{\text {eff }}}}$ & $\overline{\log g}$ & $\bar{V} \overline{\sin i}$ & $\overline{\overline{R V}}$ & $\overline{\mathrm{C} F \mathrm{PP}}$ & $\overline{\overline{C E W}}$ & $\mathrm{co}$ \\
\hline SMC5_005014 & 005529.400 & -721521.20 & 17.79 & -0.18 & 30 & $15500 \pm 1500$ & $4.2 \pm 0.2$ & $231 \pm 35$ & $155 \pm 10$ & $\bar{B} 3 \mathrm{~V}$ & B2.5III & \\
\hline SMC5_ & 0 & -72 & 17.00 & -0.19 & 40 & $21500 \pm 1600$ & $4.3 \pm 0.2$ & $105 \pm 10$ & $185 \pm 10$ & B2V & B2III & \\
\hline 095 & & -72 & 17.27 & -0.22 & 35 & $20000 \pm 2000$ & $4.2 \pm 0.2$ & \pm 39 & $182 \pm 10$ & $\mathrm{~B} 2 \mathrm{~V}$ & $.5 \mathrm{IV}$ & \\
\hline SMC5_C & 10 & -72 & 17.87 & -0.23 & 20 & $19500 \pm 2000$ & $4.3 \pm 0.2$ & $164 \pm 25$ & $168 \pm 10$ & B2V & B2IV & \\
\hline C5_005229 & 400 & $-72 \quad 1407.10$ & 17.81 & -0.13 & 20 & $17500 \pm 1700$ & $4.5 \pm 0.2$ & $1 \pm 21$ & $8 \pm 10$ & B3V & B3III & \\
\hline SMC5_013954 & .766 & -722754.67 & 16.63 & -0.12 & 70 & $15000 \pm 600$ & $3.3 \pm 0.2$ & $317 \pm 16$ & $138 \pm 10$ & B3III & B2III & $\mathrm{cl} 8$ \\
\hline SMC5_014509 & 005455.840 & -722725.50 & 17.90 & -0.05 & 20 & $15500 \pm 1500$ & $4.5 \pm 0.2$ & $174 \pm 26$ & $195 \pm 10$ & B3V & $2.5 \mathrm{III}$ & \\
\hline SMC5_014989 & 005342.820 & -722651.40 & 17.23 & -0.18 & 25 & $17000 \pm 1700$ & $3.9 \pm 0.2$ & $80 \pm 12$ & $147 \pm 10$ & B3IV & B2III & \\
\hline C5_015117 & 5340.450 & -722644.70 & 18.00 & -0.14 & 15 & $15000 \pm 1500$ & $4.2 \pm 0.2$ & $88 \pm 13$ & $147 \pm 10$ & B3V & B3III & \\
\hline SMC5_015183 & 005628.620 & $-72 \quad 2645.70$ & 15.61 & 0.02 & 55 & $10000 \pm 600$ & $3.0 \pm 0.2$ & $61 \pm 10$ & $139 \pm 10$ & A0III & B6III & $\mathrm{cl0}$ \\
\hline SMC5_015618 & 05358.460 & -722614.40 & 17.85 & -0.20 & 20 & $22000 \pm 2200$ & $4.5 \pm 0.2$ & $40 \pm 10$ & $125 \pm 10$ & $\mathrm{~B} 2 \mathrm{~V}$ & B2III & \\
\hline SMC5_016652 & 5346.212 & $-72 \quad 2510.21$ & 17.76 & -0.18 & 55 & $17500 \pm 1000$ & $4.4 \pm 0.2$ & $39 \pm 10$ & $142 \pm 10$ & B3V & B3IV & \\
\hline SMC5_016950 & 01.118 & -722450.24 & 17.50 & -0.36 & 45 & $18500 \pm 1400$ & $4.5 \pm 0.2$ & $123 \pm 12$ & $165 \pm 10$ & $\mathrm{~B} 2 \mathrm{~V}$ & B3V & \\
\hline SMC5_C & 190 & -722433.20 & 17.54 & -0.24 & 25 & $18000 \pm 1800$ & $4.4 \pm 0.2$ & $53 \pm 10$ & $128 \pm 10$ & $\mathrm{~B} 2 \mathrm{~V}$ & B2.5III & \\
\hline SMC5 & 430 & -722134.70 & 16.10 & -0.28 & 50 & $20500=$ & $3.6 \pm 0.2$ & $158 \pm 12$ & $196 \pm 10$ & B2IV & B0.5III & \\
\hline 303 & 80 & -722 & 16.14 & -0.22 & 45 & 17500 & $3.5 \pm 0.2$ & $74 \pm$ & $155 \pm 10$ & B3IV & B1.5III & \\
\hline 451 & 10 & -722 & 17.91 & -0.24 & 20 & $00 \pm 1800$ & $4.2 \pm 0.2$ & $243 \pm 36$ & $118 \pm 10$ & $\mathrm{~B} 2 \mathrm{~V}$ & B2III & cl1 \\
\hline SMC5_ & 630 & -7221 & 15.66 & -0.18 & 50 & 19000 & $5 \pm 0.2$ & 10 & $173 \pm 10$ & B2IV & B0.5III & \\
\hline 733 & .748 & -7220 & 17.93 & -0.21 & 45 & $17000 \pm$ & \pm 0.2 & 10 & $7 \pm 10$ & B3V & B3IV & \\
\hline 815 & 502 & -7220 & 16.93 & -0.13 & 65 & $11000 \pm 600$ & $1 \pm 0.2$ & $101 \pm 10$ & $124 \pm 10$ & B9III & B3III & \\
\hline 070 & 730 & -722040.10 & 17.33 & -0.22 & 30 & $17000 \pm 1700$ & $3.9 \pm 0.2$ & 10 & $0 \pm 10$ & B3IV & B2III & \\
\hline 763 & 5614.502 & $-72 \quad 1955.42$ & 17.02 & -0.24 & 60 & $17500 \pm 900$ & $3.8 \pm 0.2$ & $324 \pm 26$ & $121 \pm 10$ & B2-3IV & B1.5III & \\
\hline C5_022612 & 5331.320 & -721850.10 & 16.05 & -0.22 & 45 & $20000 \pm 1500$ & $3.8 \pm 0.2$ & $238 \pm 24$ & $150 \pm 10$ & B2IV & B1III & \\
\hline SMC5_0 & 5630.648 & $\begin{array}{llll}-72 & 18 & 12.43\end{array}$ & 17.81 & -0.27 & 40 & $18000 \pm 1400$ & $4.3 \pm 0.2$ & $91 \pm 10$ & $123 \pm 10$ & $B 2 V$ & B2III & \\
\hline 482 & 21.470 & -721746.30 & 15.22 & -0.08 & 60 & 900 & \pm 0.2 & & 10 & B2III & & \\
\hline 575 & 870 & -721740.70 & 16.47 & -0.19 & 40 & 800 & $4.0 \pm 0.2$ & & t. \pm 10 & B1V & $1 \mathrm{~V}$ & \\
\hline 556 & 860 & -7217 & 17.98 & -0.12 & 20 & 500 & 0.2 & 30 & & B3V & B3III & \\
\hline 90 & & -721 & 17.75 & -0.17 & 20 & 800 & 0.2 & & $159 \pm 10$ & B2V & B2IV & \\
\hline 164 & & -721 & 17.86 & -0.13 & 20 & 300 & 0.2 & & \pm 10 & 7IIV & B3III & \\
\hline 49 & & -72 & 15.61 & -0.27 & 60 & 000 & 0.2 & 16 & 10 & $2 \mathrm{IV}$ & B0IV & \\
\hline 88 & & -72 & 17.39 & -0.13 & 35 & 00 & .2 & & & IV & 2III & \\
\hline & & -721 & 16.29 & -0.30 & 35 & 00 & \pm 0.2 & 25 & 10 & IV & O9IV & \\
\hline 96 & 20 & -721 & 16.72 & -0.16 & 45 & 00 & $3.9 \pm 0.2$ & 13 & 10 & $3 I V$ & $32 \mathrm{IV}$ & \\
\hline 999 & 10 & -721 & 17.79 & -0.17 & 30 & 20 & \pm 0.2 & 15 & 10 & $\mathrm{~B} 2 \mathrm{~V}$ & B2IV & \\
\hline SMC5_C & 220 & -721440.40 & 16.73 & -0.19 & 40 & 400 & $4.0 \pm 0.2$ & 10 & 10 & B2V & B2III & \\
\hline 331 & 11.975 & $-72 \quad 1430.45$ & 17.10 & -0.17 & 75 & 18500 & $4.0 \pm 0.2$ & 10 & $7 \pm 10$ & B2IV & B2III & $\mathrm{cl} 6$ \\
\hline 348 & 10.520 & $-72 \quad 1432.80$ & 7.51 & -0.17 & 30 & 190 & $4.4 \pm 0.2$ & 24 & \pm 10 & B2V & $\mathrm{B} 2 \mathrm{~V}$ & \\
\hline 427 & 5328.470 & -721201.60 & 5.99 & -0.14 & 45 & 200 & $7 \pm 0.2$ & $3 \pm 20$ & $173 \pm 10$ & 2III-IV & B1III & 15 \\
\hline 283 & 5329.140 & -722645.10 & 5.66 & -0.13 & 40 & 00 & $3.5 \pm 0.2$ & 10 & \pm 10 & $32 \mathrm{III}$ & B1III & \\
\hline 332 & 20.600 & -722621.90 & 17.93 & -0.14 & 20 & $19000 \pm$ & $4.5 \pm 0.2$ & $273 \pm 41$ & $165 \pm 10$ & B2V & B1.5IV & \\
\hline 341 & .672 & -722625.49 & 16.57 & -0.19 & 80 & 00 & $3.7 \pm 0.2$ & 10 & $170=$ & $32 \mathrm{IV}$ & B1III & \\
\hline & & -72 & 7.95 & -0.13 & 20 & & 0.2 & & & B3V & $.5 \mathrm{III}$ & \\
\hline 981 & 90 & -722 & 16.17 & -0.24 & 35 & 000 & 0.2 & 19 & 10 & $2 \mathrm{IV}$ & B0.5IV & \\
\hline & & -72 & 1 & -0.18 & 65 & & .2 & & & IV & B1III & \\
\hline & & -72 & 17 & 25 & 35 & & .2 & & & $3 \mathrm{~V}$ & B2III & \\
\hline & & -72 & 40 & 0.10 & 35 & & & & & IV & B2III & \\
\hline & & -721 & 17.14 & -0.03 & 70 & & 0.2 & 10 & & $0 I V$ & B8.5III & \\
\hline & 20 & -721 & 17.31 & -0.22 & 35 & 18 & \pm 0.2 & & & $\mathrm{~B} 2 \mathrm{~V}$ & B2III & \\
\hline 564 & 17.050 & -721420.50 & 16.07 & -0.26 & 60 & 3200 & $4.1 \pm 0.2$ & 30 & 10 & O9V & & $\mathrm{ClO}$ \\
\hline 631 & .770 & $-72 \quad 1335.40$ & 17.73 & -0.08 & 20 & $12500 \pm$ & $3.8 \pm 0.2$ & $310 \pm 47$ & $151 \pm 10$ & B7IV & B3III & \\
\hline 030 & 040 & -722730.80 & 17.53 & -0.19 & 20 & 100 & $5 \pm 0.2$ & 14 & 10 & B2V & B1III & \\
\hline SMC5_0 & 5442.744 & -722725.64 & 17.26 & -0.14 & 65 & $14000 \pm 600$ & $4.0 \pm 0.2$ & $181 \pm 15$ & $121 \pm 10$ & B5IV & B3III & \\
\hline 795 & .565 & -722634.58 & 17.28 & -0.27 & 60 & $17500 \pm 800$ & $4.0 \pm 0.2$ & $49 \pm 10$ & $152 \pm 10$ & B3IV-V & $2.5 \mathrm{IV}$ & \\
\hline 182 & 373 & -722600.86 & 1714 & -0.08 & 55 & $15500+$ & $3.9 \pm 0.2$ & 11 & $140 \pm$ & B3IV & $2.5 \mathrm{III}$ & \\
\hline 323 & 21.219 & -722549.73 & 17.59 & -0.18 & 50 & $20000 \pm 1200$ & $4.3 \pm 0.2$ & $197 \pm 16$ & $147 \pm 10$ & B2V & B2IV & \\
\hline 080 & .720 & -722323.00 & 17.45 & -0.10 & 30 & $16000 \pm 1600$ & $4.2 \pm 0.2$ & $82 \pm 13$ & $151 \pm 10$ & B3V & B2III & \\
\hline SMC5_049673 & 005530.151 & -722112.79 & 17.37 & -0.25 & 55 & $18500 \pm 800$ & $4.1 \pm 0.2$ & $109 \pm 10$ & $140 \pm 10$ & $\mathrm{~B} 2 \mathrm{~V}$ & B2III & \\
\hline SMC5_0 & 22.620 & -722113.90 & 17.90 & -0.20 & 25 & $20000 \pm 2000$ & $4.4 \pm 0.2$ & $275 \pm 41$ & $140 \pm$ & $\mathrm{B} 2 \mathrm{~V}$ & B2III & \\
\hline SMC5_04 & 005359.190 & -722056.70 & 16.34 & -0.25 & 40 & $20000 \pm 1500$ & $3.9 \pm 0.2$ & $107 \pm 10$ & $155 \pm 10$ & B2IV & B1.5III & \\
\hline SMC5_049859 & 005339.290 & -722053.40 & 16.21 & -0.21 & 40 & $17500 \pm 1300$ & $3.6 \pm 0.2$ & $137 \pm 14$ & $119 \pm 10$ & B3IV & B1.5III & C17 \\
\hline
\end{tabular}


Table 1. continued.

\begin{tabular}{|c|c|c|c|c|c|c|c|c|c|c|c|c|}
\hline$\overline{\text { Star }}$ & $\overline{\alpha e(2000)}$ & $\overline{\overline{\delta(2000)}}$ & $\overline{\bar{V}}$ & $\overline{\overline{B-V}}$ & 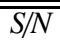 & $\overline{\overline{T_{\text {eff }}}}$ & $\overline{\log g}$ & $\overline{\bar{V} \sin i}$ & $\overline{R V}$ & $\overline{\overline{C F P}}$ & $\overline{\overline{C E W}}$ & $\mathrm{co}$ \\
\hline SMC5_050021 & 005544.471 & -722047.77 & 17.23 & -0.14 & 60 & $17500 \pm 800$ & $4.2 \pm 0.2$ & $127 \pm 10$ & $150 \pm 10$ & $\mathrm{~B} 3 \mathrm{~V}$ & B3IV & \\
\hline SMC5_ & 114 & -722 & 17.43 & -0.18 & 50 & 00 & $4.2 \pm 0.2$ & $208 \pm 16$ & $115 \pm 10$ & & B3III & \\
\hline $\mathrm{SMC5}^{-}$ & & -72 & 16.37 & -0.24 & 30 & 000 & \pm 0.2 & 24 & $165 \pm 10$ & B2IV & $1.5 \mathrm{III}$ & \\
\hline SMC5_ & 22 & -721 & 57 & 0.17 & 45 & $2000=$ & $7 \pm 0.2$ & 18 & $147 \pm 10$ & B8III-IV & B6III & \\
\hline SMC5_050904 & 740 & $\begin{array}{lll}-72 & 19 & 29.20\end{array}$ & 17.97 & 0.25 & 20 & 900 & \pm 0.2 & \pm 10 & $1 \pm 10$ & $\mathrm{~B} 2 \mathrm{~V}$ & B2III & \\
\hline SMC5_051112 & .805 & -721911.64 & 17.10 & -0.25 & 35 & $7500 \pm 1800$ & $3.9 \pm 0.2$ & $138 \pm 20$ & $126 \pm 10$ & B3IV & B1V & \\
\hline SMC5_0 & 5529.950 & -721909.90 & 15.90 & -0.25 & 45 & $500 \pm 1600$ & $3.8 \pm 0.2$ & $246 \pm 25$ & $140 \pm 10$ & B2IV & B1III & \\
\hline SMC5_051923 & 5558.105 & -721742.05 & 17.51 & -0.16 & 45 & $000 \pm 1500$ & $4.3 \pm 0.2$ & $384 \pm 38$ & $172 \pm 10$ & B2V & B2IV & \\
\hline SMC5_052147 & 5331.580 & -721715.30 & 16.48 & -0.18 & 40 & $2000 \pm 1600$ & $4.1 \pm 0.2$ & $126 \pm 13$ & $174 \pm 10$ & B2V & $1.5 \mathrm{III}$ & \\
\hline SMC5_052342 & 5551.340 & -721704.40 & 17.72 & -0.18 & 30 & $9000 \pm 1900$ & $4.4 \pm 0.2$ & $230 \pm 35$ & $147 \pm 10$ & B2V & B2III & \\
\hline SMC5_052564 & 05530.486 & -721645.74 & 17.76 & -0.16 & 50 & $0500 \pm 1300$ & $4.3 \pm 0.2$ & $71 \pm 10$ & $169 \pm 10$ & B2V & B2IV & \\
\hline SMC5_053069 & 5728.255 & -721559.33 & 17.29 & -0.23 & 50 & $8000 \pm 1100$ & $3.9 \pm 0.2$ & $50 \pm 10$ & $164 \pm 10$ & B2IV & B2IV & cl4 \\
\hline SMC5_053323 & 5611.180 & -721536.80 & 17.38 & -0.17 & 30 & $20000 \pm 2000$ & $4.3 \pm 0.2$ & $223 \pm 34$ & $211 \pm 10$ & $\mathrm{~B} 2 \mathrm{~V}$ & B2III & \\
\hline SMC5_( & 900 & -721 & 17.15 & -0.18 & 40 & $0000 \pm$ & $4.1 \pm 0.2$ & $136 \pm 18$ & $98 \pm 10$ & B2V & B1.5III & \\
\hline SMC5_( & 00 & -721 & 17.14 & -0.14 & 60 & 170 & $3.9 \pm 0.2$ & $66 \pm 10$ & $152 \pm 10$ & B3IV & B2IV & cl6 \\
\hline SMC5_( & 30 & -72 & 16.38 & -0.14 & 45 & 200 & $4.0 \pm 0.2$ & 182 & $147 \pm 10$ & $2 \mathrm{IV}$ & B1III & 6 \\
\hline SM & 40 & -72 & 17.12 & -0.14 & 30 & 175 & 0.2 & 75 & $173 \pm 10$ & B3IV & B2III & \\
\hline SMC5_C & 50 & -721426.70 & 17.70 & -0.21 & 30 & 00 & $4.1 \pm 0.2$ & $171 \pm 25$ & $177 \pm 10$ & B3V & $2.5 \mathrm{III}$ & \\
\hline SMC5_( & 991 & -72205 & 17.93 & -0.01 & 30 & 00 & $=0.2$ & 35 & $151 \pm 10$ & B3V & $2.5 \mathrm{III}$ & \\
\hline 688 & 160 & -722044.60 & 17.82 & -0.07 & 30 & 00 & 0.2 & $372 \pm 50$ & \pm 10 & B2V & B1III & \\
\hline 582 & 950 & -722759.40 & 16.29 & -0.20 & 35 & 00 & \pm 0.2 & \pm 28 & \pm 10 & B2V & $1.5 \mathrm{III}$ & 8 \\
\hline 5_064781 & 5628.110 & -722732.80 & 17.71 & -0.17 & 25 & 000 & $3 \pm 0.2$ & $57 \pm 38$ & $3 \pm 10$ & $32 \mathrm{~V}$ & B2IV & \\
\hline 5_064993 & 360 & -722653.50 & 17.93 & -0.15 & 20 & 900 & $4.5 \pm 0.2$ & \pm 28 & $6 \pm 10$ & B2V & B2III & \\
\hline SIVICS_U & 5352.592 & -722648.40 & 17.84 & -0.14 & 30 & 1600 & $4.4 \pm 0.2$ & $105 \pm 16$ & $137 \pm 10$ & B3V & B3V & \\
\hline 64 & 24.936 & -722648.43 & 16.56 & -0.17 & 80 & 00 & \pm 0.1 & $189 \pm 10$ & $157 \pm 10$ & B2IV & B1.5III & $\mathrm{cl} 0$ \\
\hline 05 & 21.462 & -722526.14 & 16.89 & -0.16 & 80 & & $=0.1$ & \pm 10 & 10 & B3V & $3 I V$ & \\
\hline & & -722 & 16.84 & -0.19 & 65 & & $=0.2$ & & & $32 \mathrm{IV}$ & B2III & \\
\hline SMC5_ & & -722 & 17.98 & -0.19 & 20 & 500 & 0.2 & 30 & $152 \pm 10$ & B3V & 3 III & \\
\hline 46 & & -721 & 17.12 & -0.23 & 55 & 00 & 0.2 & & 10 & B1V & $1 \mathrm{III}$ & \\
\hline 96 & & -721 & 17.21 & -0.17 & 50 & & 0.2 & & 10 & $3 \mathrm{IV}$ & 2 III & \\
\hline SM & & -72 & 16.80 & -0.15 & 40 & 00 & 0.2 & 24 & 10 & $\mathrm{~B} 2 \mathrm{~V}$ & 2III & C15 \\
\hline SM & & -721 & 7 & -0.18 & 50 & & 0.2 & 18 & 10 & $2 \mathrm{IV}$ & B1III & \\
\hline SM & & -721 & 17.83 & -0.21 & 30 & & .2 & 16 & 10 & $\mathrm{~V}$ & B3III & \\
\hline $\mathrm{Sl}$ & 80 & -721 & 17.81 & -0.22 & 30 & 00 & 0.2 & 22 & $=10$ & $2 \mathrm{~V}$ & $2.5 \mathrm{III}$ & \\
\hline SMC5_( & 760 & -722655.80 & 17.10 & -0.14 & 30 & 00 & \pm 0.2 & $125 \pm 19$ & 10 & $5 \mathrm{IV}$ & $2.5 \mathrm{III}$ & \\
\hline 5 5_0 & 562 & -721845.66 & 17.27 & -0.23 & 50 & 00 & \pm 0.2 & $5 \pm 11$ & $166 \pm 10$ & B3V & $2.5 \mathrm{~V}$ & \\
\hline 943 & 560 & -721631.74 & 17.42 & -0.11 & 45 & 300 & \pm 0.2 & $=16$ & 10 & $3 I V$ & 2.5III & \\
\hline SMC5_0 & 005610.006 & -722623.79 & 17.18 & -0.07 & 70 & 0 & \pm 0.2 & \pm 10 & \pm 10 & $3 I V$ & $2.5 \mathrm{III}$ & \\
\hline 305 & 5534.353 & -722130.12 & 16.88 & -0.10 & 70 & 00 & \pm 0.2 & $=19$ & \pm 10 & $1 \mathrm{IV}$ & B1IV & \\
\hline 856 & 01.880 & -721739.00 & 16.19 & -0.23 & 40 & 00 & $3.7 \pm 0.2$ & \pm 19 & \pm 10 & 2IV & $.5 \mathrm{IV}$ & \\
\hline 241 & 570 & -721 & 17.41 & -0.21 & 30 & 00 & $2 \pm 0.2$ & 27 & & $32 \mathrm{~V}$ & B2IV & \\
\hline & & -721 & 17.87 & -0.20 & 30 & & 0.2 & $=40$ & 10 & B3V & B2III & 20 \\
\hline 17 & & -722 & 17.79 & -0.23 & 25 & 00 & 0.2 & $=60$ & 10 & $\mathrm{~B} 2 \mathrm{~V}$ & B2III & \\
\hline & & -72 & 16.29 & 27 & 45 & & .2 & 12 & & IV & III & \\
\hline & & -721 & 17 & -0 & 40 & & & & & B3V & $.5 \mathrm{III}$ & \\
\hline & & -72 & & -0.04 & 20 & & & & & B3V & 3III & \\
\hline & & -721 & 16.63 & -0.07 & 75 & & .2 & 10 & & B2IV & B2III & \\
\hline & & -721 & 16.63 & -0.19 & 75 & & .1 & & & IV & $1.5 \mathrm{IV}$ & \\
\hline 15 & 950 & -721847.10 & 14.18 & -0.08 & 110 & 0 & $2.6=$ & & 10 & B8II-III & B2III & \\
\hline 021 & 600 & -722428.60 & 16.38 & -0.18 & 40 & 500 & $3.6 \pm 0.2$ & $392 \pm 39$ & $161 \pm 10$ & B2IV & B $0.5 \mathrm{~V}$ & \\
\hline & 84 & -722128.56 & 17.61 & -0.24 & 50 & 00 & \pm 0.2 & 10 & 10 & $B 2 \mathrm{~V}$ & B2.5IV & \\
\hline SMC5 0 & 550050 & $\begin{array}{lll}-72 & 19 & 23.30\end{array}$ & 15.38 & -0.10 & 60 & 00 & $.4 \pm 0.2$ & $50 \pm 10$ & $166 \pm 10$ & B3III & B1III & \\
\hline 05 & 37590 & -721455.70 & 15.12 & -0.03 & 60 & $10000 \pm$ & $2.9 \pm 0.2$ & $47 \pm 10$ & $203 \pm 10$ & A0II-III & B6III & \\
\hline 508 & 660 & -721127.30 & 16.47 & -0.19 & 40 & 205 & $3.8 \pm 0.2$ & $67 \pm 10$ & $165 \pm 10$ & B2IV & B1III & \\
\hline & 25.611 & -722222.61 & 17.23 & -0.14 & 60 & 00 & $3.8 \pm 0.2$ & $146 \pm 10$ & $144 \pm 10$ & B3IV & B2.5III & \\
\hline 033 & 5316.190 & -722208.80 & 17.88 & -0.16 & 20 & $15500 \pm 1600$ & $4.4 \pm 0.2$ & $116 \pm 17$ & $121 \pm 10$ & B3V & B2.5III & \\
\hline 5_080142 & 5513.451 & -721533.18 & 17.05 & -0.17 & 70 & $18000 \pm 700$ & $4.2 \pm 0.2$ & $59 \pm 10$ & $151 \pm 10$ & B2V & B2.5IV & \\
\hline 314 & 09.859 & -721958.80 & 17.86 & -0.26 & 50 & $20000 \pm 1$ & $4.3 \pm 0.2$ & $186 \pm 15$ & $121 \pm 10$ & B2V & B2III & \\
\hline SM & 05731.196 & -721558.83 & 17.20 & -0.10 & 50 & $16500 \pm 1000$ & $4.0 \pm 0.2$ & $161 \pm 13$ & $146 \pm 10$ & B3V & B2III & 614 \\
\hline SMC5_081871 & 005510.890 & -721527.90 & 17.33 & -0.22 & 30 & $18500 \pm 1800$ & $4.3 \pm 0.2$ & $183 \pm 28$ & $162 \pm 10$ & B2V & B2III & \\
\hline
\end{tabular}


Table 1. continued.

\begin{tabular}{|c|c|c|c|c|c|c|c|c|c|c|c|c|}
\hline$\overline{\text { Star }}$ & $\overline{\alpha \overline{\alpha(2000)}}$ & $\overline{\delta \delta(2000)}$ & $\bar{V}$ & $\overline{B-V}$ & $\overline{S / N}$ & $\overline{T_{\text {eff }}}$ & $\overline{\log g}$ & $\overline{V \sin i}$ & $\overline{\overline{R V}}$ & $\overline{\overline{C F P}}$ & $\overline{\overline{\mathrm{CEW}}}$ & com. \\
\hline SMC5_082184 & 005508.970 & -721928.00 & 17.84 & -0.25 & 25 & $17500 \pm 1700$ & $4.5 \pm 0.2$ & $211 \pm 32$ & $170 \pm 10$ & $\mathrm{~B} 2 \mathrm{~V}$ & & \\
\hline SMC5_082196 & 2.240 & -721 & 17.85 & -0.19 & 20 & $18500 \pm 1800$ & $4.4 \pm 0.2$ & 17 & $169 \pm 10$ & B2V & & \\
\hline SMC5_082379 & .780 & -72 & 17.65 & -0.17 & 25 & 1500 & $1 \pm 0.2$ & 260 & $129 \pm 10$ & B5V & $.5 \mathrm{III}$ & \\
\hline SMC5_082441 & 68 & -722426.02 & 16.81 & -0.20 & 65 & 00 & $3.4 \pm 0.2$ & 10 & $123 \pm 10$ & B8III & B6III & \\
\hline 583 & .066 & -721749.41 & 17.24 & -0.18 & 60 & $17000 \pm 800$ & $3.8 \pm 0.2$ & $316 \pm 25$ & $137 \pm 10$ & B3IV & B3V & \\
\hline SMC5_C & .190 & -721139.90 & 14.36 & -0.02 & 75 & $15500 \pm 500$ & $2.9 \pm 0.1$ & $67 \pm 10$ & $155 \pm 10$ & B3III & B0III & cl5 \\
\hline SMC5_C & 650 & -722209.70 & 16.49 & -0.23 & 40 & 18500 & $3.9 \pm 0.2$ & $149 \pm 15$ & $154 \pm 10$ & B2IV & B2.5III & \\
\hline SMC5_083593 & 005323.760 & -722232.00 & 17.42 & -0.11 & 30 & 1950 & $4.5 \pm 0.2$ & $221 \pm 33$ & $156 \pm 10$ & B2V & B2III & \\
\hline SMC5_083615 & 005324.320 & -722343.90 & 17.93 & -0.21 & 20 & 1600 & $4.5 \pm 0.2$ & $374 \pm 55$ & $158 \pm 10$ & B3V & B3III & \\
\hline SMC5_083647 & 005325.390 & $-72 \quad 1140.40$ & 17.93 & -0.32 & 35 & $16500 \pm 1700$ & $4.0 \pm 0.2$ & $159 \pm 16$ & $170 \pm 10$ & B3IV-V & B2III & c15 \\
\hline SMC5_083676 & 005326.252 & -722414.04 & 17.44 & -0.10 & 70 & $12000 \pm 1200$ & $3.6 \pm 0.2$ & $241 \pm 36$ & $113 \pm 10$ & B7III & B3III & \\
\hline SMC5_084284 & 005351.651 & -722638.51 & 16.60 & -0.12 & 60 & $17500 \pm 800$ & $3.9 \pm 0.2$ & $133 \pm 10$ & $166 \pm 10$ & B3IV-V & B2.5IV & \\
\hline SMC5_084582 & 005405.590 & -722230.30 & 15.20 & -0.03 & 50 & $10000 \pm 600$ & $3.1 \pm 0.2$ & $30 \pm 10$ & $135 \pm 10$ & A0III & B7III & \\
\hline SMC5_C & 620 & -721706.10 & 17.93 & -0.05 & 20 & $13500=$ & $3.8 \pm 0.2$ & $150 \pm 23$ & $139 \pm 10$ & B5IV & B2.5III & \\
\hline SMC5_08 & 005542.770 & -721554.20 & 17.34 & -0.27 & 35 & $24000 \pm 4200$ & $4.3 \pm 0.2$ & $241 \pm 36$ & $187 \pm 10$ & B1V & B1IV & \\
\hline SMC5_0 & 005 & -722121.99 & 17.61 & -0.11 & 45 & 15000 & $4.3 \pm 0.2$ & $284 \pm 28$ & $173 \pm 10$ & B3V & B6V & \\
\hline 07 & $00 \leq$ & -721 & 18.00 & -0.01 & 20 & 200 & \pm 0.2 & 15 & $140 \pm 10$ & B8IV & B6III & \\
\hline SM & 300 & -721 & 17.91 & -0.26 & 25 & 000 & $4.3 \pm 0.2$ & 22 & $151 \pm 10$ & $\mathrm{~B} 2 \mathrm{~V}$ & B2.5III & \\
\hline 66 & 71 & -72200 & 17.27 & -0.28 & 60 & 215 & $4.3 \pm 0.2$ & $256 \pm 20$ & $164 \pm 10$ & B2V & B2III & \\
\hline SMC5_0 & 005645.154 & -721904.56 & 17.84 & -0.43 & 40 & 1850 & $4.2 \pm 0.2$ & $136 \pm 14$ & $172 \pm 10$ & B2V & B2IV & \\
\hline SMC5_0 & 5526.900 & -721756.20 & 16.30 & -0.36 & 45 & $18000=$ & $3.8 \pm 0.2$ & $89 \pm 10$ & $158 \pm 10$ & B2IV & B2III & \\
\hline SMC5_092642 & 005643.680 & -721533.40 & 17.78 & -0.20 & 30 & $19000 \pm 1900$ & $4.5 \pm 0.2$ & $244 \pm 36$ & $186 \pm 10$ & $\mathrm{~B} 2 \mathrm{~V}$ & B2III & \\
\hline SMC5_102979 & 005450.967 & -722738.06 & 17.32 & 0.18 & 25 & $8000 \pm 800$ & $2.5 \pm 0.1$ & $16 \pm 20$ & $163 \pm 10$ & A5II-III & & cl8 \\
\hline $5 \_115576$ & 5617.477 & -721801.19 & 17.29 & -0.37 & 50 & $20000 \pm 1200$ & $3.9 \pm 0.2$ & $333 \pm 26$ & $162 \pm 10$ & B2IV & B1.5III & \\
\hline 968 & 005450.080 & -722757.50 & 17.25 & -0.32 & 40 & $18500 \pm 1400$ & $4.1 \pm 0.2$ & $177 \pm 17$ & $147 \pm 10$ & $\mathrm{~B} 2 \mathrm{~V}$ & B2III & cl8 \\
\hline SMC5_191509 & 005410.138 & -721344.69 & 17.17 & -0.21 & 70 & $14000 \pm 600$ & $3.8 \pm 0.2$ & $257 \pm 13$ & $65 \pm 10$ & B5IV & B3III & \\
\hline
\end{tabular}


C. Martayan et al.: B and Be stars in the SMC. II., Online Material $p 6$

Table 2. Parameters: $\log \left(L / L_{\odot}\right), M / M_{\odot}$ et $R / R_{\odot}$ interpolated or calculated for our sample of $\mathrm{O}, \mathrm{B}, \mathrm{A}$ stars in the SMC from HR diagrams published in Schaller et al. (1992) for $Z=0.001$.

\begin{tabular}{|c|c|c|c|c|}
\hline$\overline{\text { Star }}$ & $\overline{\log \left(L / L_{\odot}\right)}$ & $\overline{\bar{M} / M_{\odot}}$ & $\overline{R / R_{\odot}}$ & age (Myears) \\
\hline SMC5_000351 & $2.4 \pm 0.4$ & $3.4 \pm 0.5$ & $1.9 \pm 0.3$ & $125 \pm 6$ \\
\hline SMC5_000398 & $4.2 \pm 0.4$ & $9.7 \pm 0.5$ & $24.8 \pm 2.5$ & $26 \pm 3$ \\
\hline SMC5_000432 & $3.1 \pm 0.4$ & $4.5 \pm 0.5$ & $5.0 \pm 1.0$ & $119 \pm 6$ \\
\hline SMC5_000621 & $3.1 \pm 0.4$ & $5.2 \pm 0.5$ & $3.1 \pm 0.5$ & $65 \pm 6$ \\
\hline SMC5_000660 & $2.6 \pm 0.4$ & $3.8 \pm 0.5$ & $2.5 \pm 0.5$ & $137 \pm 6$ \\
\hline SMC5_000670 & $3.3 \pm 0.4$ & $5.8 \pm 0.5$ & $4.4 \pm 0.5$ & $67 \pm 6$ \\
\hline SMC5_000810 & - & - & - & - \\
\hline SMC5_000889 & $2.4 \pm 0.4$ & $2.8 \pm 0.5$ & $4.0 \pm 0.5$ & $25 \pm 3$ \\
\hline SMC5_000924 & $2.9 \pm 0.4$ & $5.0 \pm 0.5$ & $2.4 \pm 0.5$ & $54 \pm 6$ \\
\hline SMC5_000959 & $3.2 \pm 0.4$ & $5.4 \pm 0.5$ & $4.0 \pm 0.5$ & $73 \pm 6$ \\
\hline SMC5_002782 & $3.9 \pm 0.4$ & $8.8 \pm 0.5$ & $4.5 \pm 0.5$ & $71 \pm 3$ \\
\hline SMC5_003118 & $3.6 \pm 0.4$ & $6.6 \pm 0.5$ & $5.4 \pm 1.0$ & $22 \pm 3$ \\
\hline SMC5_003175 & $3.5 \pm 0.4$ & $6.9 \pm 0.5$ & $4.1 \pm 0.5$ & $41 \pm 3$ \\
\hline SMC5_003195 & $2.4 \pm 0.4$ & $3.7 \pm 0.5$ & $1.8 \pm 0.3$ & $13 \pm 3$ \\
\hline SMC5_003292 & $3.2 \pm 0.4$ & $5.5 \pm 0.5$ & $3.8 \pm 0.5$ & $62 \pm 3$ \\
\hline SMC5_003310 & $3.4 \pm 0.4$ & $6.8 \pm 0.5$ & $2.9 \pm 0.5$ & $137 \pm 6$ \\
\hline SMC5_003335 & $2.4 \pm 0.4$ & $3.6 \pm 0.5$ & $1.8 \pm 0.3$ & $104 \pm 6$ \\
\hline SMC5_003739 & $4.5 \pm 0.4$ & $11.0 \pm 1.0$ & $30.6 \pm 2.5$ & $103 \pm 6$ \\
\hline SMC5_003809 & $2.1 \pm 0.4$ & $3.0 \pm 0.5$ & $1.6 \pm 0.3$ & $13 \pm 3$ \\
\hline SMC5_003855 & $4.7 \pm 0.4$ & $14.8 \pm 1.0$ & $13.2 \pm 1.5$ & $43 \pm 3$ \\
\hline SMC5_003910 & $4.4 \pm 0.4$ & $11.6 \pm 1.0$ & $15.1 \pm 1.5$ & $44 \pm 3$ \\
\hline SMC5_003942 & $3.2 \pm 0.4$ & $5.4 \pm 0.5$ & $3.9 \pm 0.5$ & $108 \pm 6$ \\
\hline SMC5_003998 & $2.5 \pm 0.4$ & $3.0 \pm 0.5$ & $3.3 \pm 0.5$ & $17 \pm 3$ \\
\hline SMC5_003999 & $3.6 \pm 0.4$ & $6.2 \pm 0.5$ & $7.5 \pm 1.0$ & $32 \pm 3$ \\
\hline SMC5_004025 & $2.4 \pm 0.4$ & $3.3 \pm 0.5$ & $2.1 \pm 0.5$ & $64 \pm 6$ \\
\hline SMC5_004034 & $3.9 \pm 0.4$ & $8.1 \pm 0.5$ & $8.4 \pm 1.0$ & $77 \pm 6$ \\
\hline SMC5_004044 & $3.1 \pm 0.4$ & $5.0 \pm 0.5$ & $3.8 \pm 0.5$ & $71 \pm 6$ \\
\hline SMC5_004102 & $3.0 \pm 0.4$ & $4.2 \pm 0.5$ & $12.3 \pm 1.5$ & $22 \pm 3$ \\
\hline SMC5_004107 & $3.2 \pm 0.4$ & $4.8 \pm 0.5$ & $4.8 \pm 0.5$ & $38 \pm 3$ \\
\hline SMC5_004133 & $3.0 \pm 0.4$ & $4.1 \pm 0.5$ & $8.4 \pm 1.0$ & $37 \pm 3$ \\
\hline SMC5_004135 & $3.1 \pm 0.4$ & $4.7 \pm 0.5$ & $4.4 \pm 0.5$ & $82 \pm 6$ \\
\hline SMC5_004149 & $2.5 \pm 0.4$ & $3.2 \pm 0.5$ & $3.5 \pm 0.5$ & $94 \pm 6$ \\
\hline SMC5_004153 & $3.3 \pm 0.4$ & $5.6 \pm 0.5$ & $4.0 \pm 0.5$ & $129 \pm 6$ \\
\hline SMC5_004171 & $2.8 \pm 0.4$ & $4.2 \pm 0.5$ & $2.6 \pm 0.5$ & $42 \pm 6$ \\
\hline SMC5_004198 & $4.8 \pm 0.4$ & $15.0 \pm 1.0$ & $13.7 \pm 1.5$ & $177 \pm 10$ \\
\hline SMC5_004203 & $3.1 \pm 0.4$ & $5.5 \pm 0.5$ & $2.6 \pm 0.5$ & $41 \pm 3$ \\
\hline SMC5_004263 & $2.9 \pm 0.4$ & $4.3 \pm 0.5$ & $3.7 \pm 0.5$ & $59 \pm 6$ \\
\hline SMC5_004326 & $3.7 \pm 0.4$ & $8.1 \pm 0.5$ & $4.0 \pm 0.5$ & $62 \pm 6$ \\
\hline SMC5_004381 & $2.9 \pm 0.4$ & $5.0 \pm 0.5$ & $2.4 \pm 0.5$ & $76 \pm 6$ \\
\hline SMC5_004413 & $3.2 \pm 0.4$ & $4.7 \pm 0.5$ & $8.8 \pm 1.0$ & $115 \pm 6$ \\
\hline SMC5_004465 & $2.6 \pm 0.4$ & $3.9 \pm 0.5$ & $2.3 \pm 0.5$ & $112 \pm 6$ \\
\hline SMC5_004502 & $4.0 \pm 0.4$ & $8.6 \pm 0.5$ & $7.6 \pm 1.0$ & $197 \pm 10$ \\
\hline SMC5_004506 & $3.5 \pm 0.4$ & $6.1 \pm 0.5$ & $4.6 \pm 0.5$ & $135 \pm 6$ \\
\hline SMC5_004534 & $3.4 \pm 0.4$ & $5.5 \pm 0.5$ & $7.9 \pm 1.0$ & $85 \pm 6$ \\
\hline SMC5_004591 & $2.8 \pm 0.4$ & $4.4 \pm 0.5$ & $2.3 \pm 0.5$ & $35 \pm 3$ \\
\hline SMC5_004695 & $4.4 \pm 0.4$ & $11.0 \pm 1.0$ & $14.2 \pm 1.5$ & $61 \pm 6$ \\
\hline SMC5_004700 & $3.9 \pm 0.4$ & $7.8 \pm 0.5$ & $7.3 \pm 1.0$ & $91 \pm 6$ \\
\hline SMC5_004718 & $2.6 \pm 0.4$ & $4.2 \pm 0.5$ & $1.9 \pm 0.5$ & $40 \pm 3$ \\
\hline SMC5_004872 & $2.5 \pm 0.4$ & $3.8 \pm 0.5$ & $2.0 \pm 0.5$ & $112 \pm 6$ \\
\hline SMC5_004885 & $2.9 \pm 0.4$ & $4.4 \pm 0.5$ & $2.8 \pm 0.5$ & $54 \pm 6$ \\
\hline SMC5_004947 & $2.5 \pm 0.4$ & $3.6 \pm 0.5$ & $2.1 \pm 0.5$ & $30 \pm 3$ \\
\hline SMC5_004988 & $3.8 \pm 0.4$ & $7.4 \pm 0.5$ & $6.6 \pm 1.0$ & $52 \pm 6$ \\
\hline SMC5_005014 & $2.5 \pm 0.4$ & $3.5 \pm 0.5$ & $2.5 \pm 0.5$ & $24 \pm 3$ \\
\hline SMC5_005090 & $3.2 \pm 0.4$ & $5.9 \pm 0.5$ & $2.8 \pm 0.5$ & $57 \pm 6$ \\
\hline SMC5_005095 & $3.1 \pm 0.4$ & $5.3 \pm 0.5$ & $3.0 \pm 0.5$ & $80 \pm 6$ \\
\hline SMC5_005215 & $2.9 \pm 0.4$ & $4.7 \pm 0.5$ & $2.5 \pm 0.5$ & $64 \pm 6$ \\
\hline SMC5_005229 & $2.5 \pm 0.4$ & $3.8 \pm 0.5$ & $1.9 \pm 0.3$ & $88 \pm 6$ \\
\hline SMC5_013954 & $3.6 \pm 0.4$ & $6.0 \pm 0.5$ & $9.2 \pm 1.0$ & $28 \pm 3$ \\
\hline
\end{tabular}


Table 2. continued.

\begin{tabular}{|c|c|c|c|c|}
\hline$\overline{\text { Star }}$ & $\overline{\log \left(L / L_{\odot}\right)}$ & $\overline{\bar{M} / M_{\odot}}$ & $\overline{\bar{R} / R_{\odot}}$ & "age (Myears) \\
\hline SMC5_014509 & $2.1 \pm 0.4$ & $3.0 \pm 0.5$ & $1.6 \pm 0.3$ & $108 \pm 6$ \\
\hline SMC5_014989 & $3.0 \pm 0.4$ & $4.5 \pm 0.5$ & $3.8 \pm 0.5$ & $24 \pm 3$ \\
\hline SMC5_015117 & $2.5 \pm 0.4$ & $3.4 \pm 0.5$ & $2.6 \pm 0.5$ & $28 \pm 3$ \\
\hline SMC5 015183 & $3.0 \pm 0.4$ & $4.2 \pm 0.5$ & $10.6 \pm 1.0$ & $29 \pm 3$ \\
\hline SMC5 015618 & $3.0 \pm 0.4$ & $5.6 \pm 0.5$ & $2.2 \pm 0.5$ & $16 \pm 3$ \\
\hline SMC5_016652 & $2.5 \pm 0.4$ & $3.7 \pm 0.5$ & $2.0 \pm 0.5$ & $100 \pm 6$ \\
\hline SMC5_016950 & $2.6 \pm 0.4$ & $4.0 \pm 0.5$ & $1.9 \pm 0.3$ & $31 \pm 3$ \\
\hline SMC5_017228 & $2.6 \pm 0.4$ & $4.0 \pm 0.5$ & $2.2 \pm 0.5$ & $52 \pm 6$ \\
\hline SMC5_020135 & $4.0 \pm 0.4$ & $8.3 \pm 0.5$ & $7.8 \pm 1.0$ & $77 \pm 6$ \\
\hline SMC5 020303 & $3.6 \pm 0.4$ & $6.3 \pm 0.5$ & $7.1 \pm 1.0$ & $52 \pm 6$ \\
\hline SMC5 020451 & $2.9 \pm 0.4$ & $4.5 \pm 0.5$ & $2.7 \pm 0.5$ & $43 \pm 6$ \\
\hline SMC5_020672 & $3.9 \pm 0.4$ & $7.7 \pm 0.5$ & $8.2 \pm 1.0$ & $49 \pm 6$ \\
\hline SMC5_020733 & $2.5 \pm 0.4$ & $3.6 \pm 0.5$ & $2.0 \pm 0.5$ & $45 \pm 6$ \\
\hline SMC5_020815 & $3.1 \pm 0.4$ & $4.3 \pm 0.5$ & $9.6 \pm 1.0$ & $203 \pm 10$ \\
\hline SMC5_021070 & $3.0 \pm 0.4$ & $4.5 \pm 0.5$ & $4.0 \pm 0.5$ & $58 \pm 6$ \\
\hline SMC5_021763 & $3.3 \pm 0.4$ & $5.3 \pm 0.5$ & $5.0 \pm 1.0$ & $47 \pm 6$ \\
\hline SMC5_022612 & $3.6 \pm 0.4$ & $6.6 \pm 0.5$ & $5.5 \pm 1.0$ & $83 \pm 6$ \\
\hline SMC5 023315 & $2.7 \pm 0.4$ & $4.1 \pm 0.5$ & $2.4 \pm 0.5$ & $88 \pm 6$ \\
\hline SMC5_023482 & $4.1 \pm 0.4$ & $8.8 \pm 0.5$ & $11.1 \pm 1.5$ & $93 \pm 6$ \\
\hline SMC5_023575 & $4.0 \pm 0.4$ & $9.5 \pm 0.5$ & $5.4 \pm 1.0$ & $26 \pm 3$ \\
\hline SMC5_023656 & $2.3 \pm 0.4$ & $3.3 \pm 0.5$ & $1.7 \pm 0.3$ & $14 \pm 3$ \\
\hline SMC5_024390 & $2.9 \pm 0.4$ & $4.4 \pm 0.5$ & $2.6 \pm 0.5$ & $26 \pm 3$ \\
\hline SMC5_024464 & $2.4 \pm 0.4$ & $2.8 \pm 0.5$ & $3.3 \pm 0.5$ & $57 \pm 6$ \\
\hline SMC5 024949 & $4.1 \pm 0.4$ & $9.1 \pm 0.5$ & $9.3 \pm 1.0$ & $69 \pm 6$ \\
\hline SMC5_025288 & $3.1 \pm 0.4$ & $4.6 \pm 0.5$ & $3.7 \pm 0.5$ & $64 \pm 6$ \\
\hline SMC5_025394 & $4.1 \pm 0.4$ & $9.0 \pm 0.5$ & $5.9 \pm 1.0$ & $51 \pm 6$ \\
\hline SMC5_025596 & $3.1 \pm 0.4$ & $4.8 \pm 0.5$ & $4.0 \pm 0.5$ & $42 \pm 3$ \\
\hline SMC5_025999 & $2.9 \pm 0.4$ & $4.9 \pm 0.5$ & $2.5 \pm 0.5$ & $58 \pm 6$ \\
\hline SMC5_026256 & $3.2 \pm 0.4$ & $5.2 \pm 0.5$ & $3.8 \pm 0.5$ & $60 \pm 6$ \\
\hline SMC5_026331 & $3.2 \pm 0.4$ & $5.0 \pm 0.5$ & $3.9 \pm 0.5$ & $110 \pm 6$ \\
\hline SMC5_026348 & $2.9 \pm 0.4$ & $4.8 \pm 0.5$ & $2.4 \pm 0.5$ & $35 \pm 3$ \\
\hline SMC5_028427 & $3.8 \pm 0.4$ & $7.3 \pm 0.5$ & $6.6 \pm 1.0$ & $57 \pm 6$ \\
\hline SMC5_037283 & $3.7 \pm 0.4$ & $6.8 \pm 0.5$ & $7.6 \pm 1.0$ & $55 \pm 6$ \\
\hline SMC5_037332 & $2.7 \pm 0.4$ & $4.4 \pm 0.5$ & $2.1 \pm 0.5$ & $44 \pm 6$ \\
\hline SMC5_037341 & $3.9 \pm 0.4$ & $7.8 \pm 0.5$ & $7.1 \pm 0.5$ & $126 \pm 6$ \\
\hline SMC5_037384 & $2.3 \pm 0.4$ & $3.1 \pm 0.5$ & $2.1 \pm 0.5$ & $61 \pm 6$ \\
\hline SMC5_037981 & $3.6 \pm 0.4$ & $6.4 \pm 0.5$ & $5.0 \pm 1.0$ & $45 \pm 6$ \\
\hline SMC5 038033 & $3.7 \pm 0.4$ & $6.9 \pm 0.5$ & $6.0 \pm 1.0$ & $155 \pm 6$ \\
\hline SMC5 038144 & $2.6 \pm 0.4$ & $3.8 \pm 0.5$ & $2.5 \pm 0.5$ & $87 \pm 6$ \\
\hline SMC5_038311 & $3.2 \pm 0.4$ & $5.0 \pm 0.5$ & $4.3 \pm 0.5$ & $65 \pm 6$ \\
\hline SMC5_038423 & $1.9 \pm 0.4$ & $2.1 \pm 0.5$ & $3.1 \pm 0.5$ & $128 \pm 6$ \\
\hline SMC5_038530 & $3.1 \pm 0.4$ & $4.8 \pm 0.5$ & $3.4 \pm 0.5$ & $49 \pm 6$ \\
\hline SMC5_038564 & $4.1 \pm 0.4$ & $9.7 \pm 0.5$ & $6.6 \pm 1.0$ & $41 \pm 3$ \\
\hline SMC5_038631 & $2.4 \pm 0.4$ & $2.9 \pm 0.5$ & $3.6 \pm 0.5$ & $177 \pm 10$ \\
\hline SMC5_045030 & $3.0 \pm 0.4$ & $5.5 \pm 0.5$ & $2.2 \pm 0.5$ & $43 \pm 3$ \\
\hline SMC5_045148 & $2.5 \pm 0.4$ & $3.3 \pm 0.5$ & $3.2 \pm 0.5$ & $46 \pm 3$ \\
\hline SMC5_045795 & $3.0 \pm 0.4$ & $4.7 \pm 0.5$ & $3.6 \pm 0.5$ & $60 \pm 6$ \\
\hline SMC5_046182 & $2.9 \pm 0.4$ & $3.9 \pm 0.5$ & $3.9 \pm 0.5$ & $38 \pm 3$ \\
\hline SMC5_046323 & $3.0 \pm 0.4$ & $5.0 \pm 0.5$ & $2.6 \pm 0.5$ & $42 \pm 3$ \\
\hline SMC5_048080 & $2.5 \pm 0.4$ & $3.5 \pm 0.5$ & $2.3 \pm 0.5$ & $169 \pm 6$ \\
\hline SMC5 049673 & $3.1 \pm 0.4$ & $4.9 \pm 0.5$ & $3.3 \pm 0.5$ & $107 \pm 6$ \\
\hline SMC5 049695 & $2.8 \pm 0.4$ & $4.7 \pm 0.5$ & $2.2 \pm 0.5$ & $45 \pm 3$ \\
\hline SMC5_049858 & $3.6 \pm 0.4$ & $6.4 \pm 0.5$ & $4.9 \pm 1.0$ & $80 \pm 6$ \\
\hline SMC5_049859 & $3.5 \pm 0.4$ & $5.9 \pm 0.5$ & $6.3 \pm 1.0$ & $57 \pm 6$ \\
\hline SMC5_050021 & $2.8 \pm 0.4$ & $4.0 \pm 0.5$ & $2.8 \pm 0.5$ & $71 \pm 6$ \\
\hline SMC5_050309 & $2.3 \pm 0.4$ & $3.1 \pm 0.5$ & $2.3 \pm 0.5$ & $67 \pm 6$ \\
\hline SMC5_050662 & $3.5 \pm 0.4$ & $6.1 \pm 0.5$ & $4.6 \pm 0.5$ & $200 \pm 20$ \\
\hline SMC5_050882 & $2.5 \pm 0.4$ & $2.9 \pm 0.5$ & $4.2 \pm 0.5$ & $27 \pm 3$ \\
\hline SMC5_050904 & $2.7 \pm 0.4$ & $4.2 \pm 0.5$ & $2.0 \pm 0.5$ & $74 \pm 6$ \\
\hline SMC5_051112 & $3.2 \pm 0.4$ & $5.0 \pm 0.5$ & $4.3 \pm 0.5$ & $112 \pm 6$ \\
\hline
\end{tabular}


Table 2. continued.

\begin{tabular}{|c|c|c|c|c|}
\hline$\overline{\text { Star }}$ & $\overline{\log \left(L / L_{\odot}\right)}$ & $\overline{\bar{M} / M_{\odot}}$ & $\overline{R / R} / R_{\odot}$ & "age (Myears) \\
\hline SMC5_051147 & $3.6 \pm 0.4$ & $6.8 \pm 0.5$ & $5.4 \pm 1.0$ & $143 \pm 6$ \\
\hline SMC5_051923 & $3.0 \pm 0.4$ & $4.9 \pm 0.5$ & $2.6 \pm 0.5$ & $50 \pm 6$ \\
\hline SMC5_052147 & $3.5 \pm 0.4$ & $6.8 \pm 0.5$ & $4.0 \pm 0.5$ & $204 \pm 20$ \\
\hline SMC5_052342 & $2.7 \pm 0.4$ & $4.3 \pm 0.5$ & $2.1 \pm 0.5$ & $248 \pm 20$ \\
\hline SMC5_052564 & $3.0 \pm 0.4$ & $5.2 \pm 0.5$ & $2.6 \pm 0.5$ & $324 \pm 20$ \\
\hline SMC5_053069 & $3.1 \pm 0.4$ & $4.8 \pm 0.5$ & $3.9 \pm 0.5$ & $74 \pm 6$ \\
\hline SMC5_053323 & $3.1 \pm 0.4$ & $5.1 \pm 0.5$ & $2.8 \pm 0.5$ & $37 \pm 3$ \\
\hline SMC5_053563 & $3.2 \pm 0.4$ & $5.7 \pm 0.5$ & $3.4 \pm 0.5$ & $86 . \pm 6$ \\
\hline SMC5_053746 & $3.1 \pm 0.4$ & $4.6 \pm 0.5$ & $4.1 \pm 0.5$ & $119 \pm 6$ \\
\hline SMC5_053755 & $3.4 \pm 0.4$ & $6.1 \pm 0.5$ & $4.3 \pm 0.5$ & $65 \pm 6$ \\
\hline SMC5_053967 & $3.0 \pm 0.4$ & $4.6 \pm 0.5$ & $3.7 \pm 0.5$ & $137 \pm 6$ \\
\hline SMC5_054028 & $2.8 \pm 0.4$ & $4.2 \pm 0.5$ & $2.9 \pm 0.5$ & $67 \pm 6$ \\
\hline SMC5_061681 & $2.4 \pm 0.4$ & $3.4 \pm 0.5$ & $2.0 \pm 0.5$ & $42 \pm 3$ \\
\hline SMC5_061688 & $2.8 \pm 0.4$ & $4.7 \pm 0.5$ & $2.1 \pm 0.5$ & $81 \pm 6$ \\
\hline SMC5_064582 & $3.3 \pm 0.4$ & $5.9 \pm 0.5$ & $3.5 \pm 0.5$ & $65 \pm 6$ \\
\hline SMC5_064781 & $3.0 \pm 0.4$ & $5.0 \pm 0.5$ & $2.6 \pm 0.5$ & $69 \pm 6$ \\
\hline SMC5_064993 & $2.7 \pm 0.4$ & $4.4 \pm 0.5$ & $2.0 \pm 0.5$ & $25 \pm 3$ \\
\hline SMC5_065022 & $2.4 \pm 0.4$ & $3.4 \pm 0.5$ & $1.9 \pm 0.3$ & $73 \pm 6$ \\
\hline SMC5_065064 & $3.5 \pm 0.4$ & $6.3 \pm 0.5$ & $5.0 \pm 1.0$ & $294 \pm 10$ \\
\hline SMC5_065505 & $2.8 \pm 0.4$ & $4.1 \pm 0.5$ & $2.8 \pm 0.5$ & $163 \pm 10$ \\
\hline SMC5_066230 & $3.3 \pm 0.4$ & $5.7 \pm 0.5$ & $4.5 \pm 0.5$ & $83 \pm 6$ \\
\hline SMC5_067093 & $2.2 \pm 0.4$ & $3.1 \pm 0.5$ & $1.7 \pm 0.3$ & $99 \pm 6$ \\
\hline SMC5_067446 & $3.6 \pm 0.4$ & $7.5 \pm 0.5$ & $3.4 \pm 0.5$ & $269 \pm 10$ \\
\hline SMC5_067596 & $3.2 \pm 0.4$ & $4.8 \pm 0.5$ & $5.2 \pm 1.0$ & $70 \pm 6$ \\
\hline SMC5_067779 & $3.2 \pm 0.4$ & $5.7 \pm 0.5$ & $3.5 \pm 0.5$ & $124 \pm 6$ \\
\hline SMC5_068593 & $3.8 \pm 0.4$ & $7.1 \pm 0.5$ & $7.3 \pm 1.0$ & $27 \pm 3$ \\
\hline SMC5_068678 & $2.3 \pm 0.4$ & $3.3 \pm 0.5$ & $2.0 \pm 0.5$ & $101 \pm 6$ \\
\hline SMC5_068752 & $2.6 \pm 0.4$ & $4.0 \pm 0.5$ & $2.2 \pm 0.5$ & $66 \pm 6$ \\
\hline SMC5_071616 & $2.7 \pm 0.4$ & $3.4 \pm 0.5$ & $3.5 \pm 0.5$ & $86 \pm 6$ \\
\hline SMC5_071876 & $2.7 \pm 0.4$ & $4.1 \pm 0.5$ & $2.5 \pm 0.5$ & $49 \pm 6$ \\
\hline SMC5_071943 & $3.1 \pm 0.4$ & $4.5 \pm 0.5$ & $4.2 \pm 0.5$ & $116 \pm 6$ \\
\hline SMC5_073584 & $2.9 \pm 0.4$ & $4.0 \pm 0.5$ & $4.0 \pm 0.5$ & $131 \pm 6$ \\
\hline SMC5_074305 & $3.8 \pm 0.4$ & $7.9 \pm 0.5$ & $4.9 \pm 1.0$ & $81 \pm 6$ \\
\hline SMC5_074856 & $3.5 \pm 0.4$ & $6.1 \pm 0.5$ & $5.9 \pm 1.0$ & $91 \pm 6$ \\
\hline SMC5_075241 & $3.1 \pm 0.4$ & $5.2 \pm 0.5$ & $3.1 \pm 0.5$ & $87 \pm 6$ \\
\hline SMC5_075287 & $2.7 \pm 0.4$ & $3.9 \pm 0.5$ & $2.7 \pm 0.5$ & $92 \pm 6$ \\
\hline SMC5_077517 & $2.9 \pm 0.4$ & $4.9 \pm 0.5$ & $2.4 \pm 0.5$ & $38 \pm 3$ \\
\hline SMC5_077609 & $3.8 \pm 0.4$ & $7.5 \pm 0.5$ & $6.5 \pm 1.0$ & $137 \pm 6$ \\
\hline SMC5_077659 & $2.7 \pm 0.4$ & $3.8 \pm 0.5$ & $3.0 \pm 0.5$ & $783 \pm 20$ \\
\hline SMC5_077666 & $2.4 \pm 0.4$ & $3.3 \pm 0.5$ & $2.2 \pm 0.5$ & $245 \pm 10$ \\
\hline SMC5_077670 & $3.3 \pm 0.4$ & $5.6 \pm 0.5$ & $4.5 \pm 0.5$ & $98 \pm 6$ \\
\hline SMC5_077712 & $3.6 \pm 0.4$ & $6.5 \pm 0.5$ & $5.0 \pm 1.0$ & $158 \pm 6$ \\
\hline SMC5_078415 & $3.8 \pm 0.4$ & $7.0 \pm 0.5$ & $22.1 \pm 2.0$ & $81 \pm 6$ \\
\hline SMC5_079021 & $3.9 \pm 0.4$ & $7.9 \pm 0.5$ & $7.8 \pm 1.0$ & $231 \pm 10$ \\
\hline SMC5_079166 & $3.0 \pm 0.4$ & $4.6 \pm 0.5$ & $3.2 \pm 0.5$ & $314 \pm 10$ \\
\hline SMC5_079264 & $3.8 \pm 0.4$ & $7.4 \pm 0.5$ & $9.5 \pm 1.0$ & $86 \pm 6$ \\
\hline SMC5_079405 & $3.2 \pm 0.4$ & $4.7 \pm 0.5$ & $13.2 \pm 1.5$ & $86 \pm 6$ \\
\hline SMC5_079508 & $3.7 \pm 0.4$ & $7.0 \pm 0.5$ & $5.9 \pm 1.0$ & $100 \pm 6$ \\
\hline SMC5_080028 & $3.0 \pm 0.4$ & $4.1 \pm 0.5$ & $4.2 \pm 0.5$ & $60 \pm 6$ \\
\hline SMC5_080033 & $2.2 \pm 0.4$ & $3.0 \pm 0.5$ & $1.8 \pm 0.3$ & $54 \pm 6$ \\
\hline SMC5_080142 & $2.8 \pm 0.4$ & $4.2 \pm 0.5$ & $2.6 \pm 0.5$ & $48 \pm 6$ \\
\hline SMC5_080814 & $3.0 \pm 0.4$ & $5.0 \pm 0.5$ & $2.7 \pm 0.5$ & $57 \pm 6$ \\
\hline SMC5_081252 & $2.9 \pm 0.4$ & $4.2 \pm 0.5$ & $3.3 \pm 0.5$ & $100 \pm 6$ \\
\hline SMC5_081871 & $2.8 \pm 0.4$ & $4.4 \pm 0.5$ & $2.5 \pm 0.5$ & $112 \pm 6$ \\
\hline SMC5_082184 & $2.5 \pm 0.4$ & $3.8 \pm 0.5$ & $1.9 \pm 0.3$ & $119 \pm 6$ \\
\hline SMC5_082196 & $2.7 \pm 0.4$ & $4.2 \pm 0.5$ & $2.1 \pm 0.5$ & $60 \pm 6$ \\
\hline SMC5_082379 & $2.6 \pm 0.4$ & $3.5 \pm 0.5$ & $2.9 \pm 0.5$ & $111 \pm 6$ \\
\hline SMC5_082441 & $2.8 \pm 0.4$ & $3.6 \pm 0.5$ & $6.4 \pm 0.5$ & $72 \pm 6$ \\
\hline SMC5_082583 & $3.2 \pm 0.4$ & $4.9 \pm 0.5$ & $4.7 \pm 0.5$ & $26 \pm 3$ \\
\hline SMC5_082923 & $4.2 \pm 0.4$ & $9.6 \pm 0.5$ & $18.5 \pm 1.5$ & $101 \pm 6$ \\
\hline
\end{tabular}


C. Martayan et al.: B and Be stars in the SMC. II., Online Material p 9

Table 2. continued.

\begin{tabular}{ccccc}
\hline \hline Star & $\log \left(L / L_{\odot}\right)$ & $M / M_{\odot}$ & $R / R_{\odot}$ & age (Myears) \\
\hline SMC5_082987 & $3.3 \pm 0.4$ & $5.6 \pm 0.5$ & $4.5 \pm 0.5$ & $100 \pm 6$ \\
SMC5_083593 & $2.7 \pm 0.4$ & $4.4 \pm 0.5$ & $2.0 \pm 0.5$ & $118 \pm 6$ \\
SMC5_083615 & $2.3 \pm 0.4$ & $3.2 \pm 0.5$ & $1.8 \pm 0.5$ & $147 \pm 6$ \\
SMC5_083647 & $2.9 \pm 0.4$ & $4.0 \pm 0.5$ & $3.4 \pm 0.5$ & $155 \pm 6$ \\
SMC5_083676 & $2.7 \pm 0.4$ & $3.3 \pm 0.5$ & $5.0 \pm 1.0$ & $72 \pm 6$ \\
SMC5_084284 & $3.1 \pm 0.4$ & $4.8 \pm 0.5$ & $4.2 \pm 0.5$ & $57 \pm 6$ \\
SMC5_084582 & $2.8 \pm 0.4$ & $3.6 \pm 0.5$ & $8.7 \pm 1.0$ & $138 \pm 6$ \\
SMC5_086155 & $2.6 \pm 0.4$ & $3.3 \pm 0.5$ & $3.7 \pm 0.5$ & $99 \pm 6$ \\
SMC5_086367 & $3.5 \pm 0.4$ & $7.3 \pm 0.5$ & $3.2 \pm 0.5$ & $59 \pm 6$ \\
SMC5_086663 & $2.3 \pm 0.4$ & $3.1 \pm 0.5$ & $2.1 \pm 0.5$ & $124 \pm 6$ \\
SMC5_086807 & $2.5 \pm 0.4$ & $2.9 \pm 0.5$ & $4.1 \pm 0.5$ & $209 \pm 10$ \\
SMC5_087022 & $2.9 \pm 0.4$ & $4.8 \pm 0.5$ & $2.4 \pm 0.5$ & $94 \pm 6$ \\
SMC5_087066 & $3.3 \pm 0.4$ & $6.0 \pm 0.5$ & $3.0 \pm 0.5$ & $247 \pm 10$ \\
SMC5_092257 & $2.9 \pm 0.4$ & $4.6 \pm 0.5$ & $2.8 \pm 0.5$ & $100 \pm 6$ \\
SMC5_092393 & $3.4 \pm 0.4$ & $5.7 \pm 0.5$ & $5.2 \pm 1.0$ & $197 \pm 10$ \\
SMC5_092642 & $2.7 \pm 0.4$ & $4.4 \pm 0.5$ & $2.0 \pm 0.5$ & $45 \pm 6$ \\
SMC5_102979 & $3.1 \pm 0.4$ & $4.0 \pm 0.5$ & $18.7 \pm 1.5$ & $130 \pm 10$ \\
SMC5_115576 & $3.5 \pm 0.4$ & $6.4 \pm 0.5$ & $4.6 \pm 0.5$ & $187 \pm 10$ \\
SMC5_188968 & $3.0 \pm 0.4$ & $4.8 \pm 0.5$ & $3.2 \pm 0.5$ & $54 \pm 6$ \\
SMC5_191509 & $2.7 \pm 0.4$ & $3.6 \pm 0.5$ & $4.1 \pm 0.5$ & $206 \pm 10$ \\
\hline
\end{tabular}


Table 3. Apparent fundamental parameters for Be stars in the SMC. The name, coordinates $(\alpha(2000), \delta(2000)), V$ magnitude and $(B-V)$ colour index of stars are taken from the EIS catalogues, except for 2 stars identified by our catalogue number of emission line stars (MHF[SX]XXXXX). The effective temperature $T_{\text {eff }}$ is given in $\mathrm{K}, \log g$ in dex, $V \sin i$ in $\mathrm{km} \mathrm{s}^{-1}$ and $R V$ in $\mathrm{km} \mathrm{s}^{-1}$. For each parameter the $1 \sigma$ error is given. The abbreviation "CFP" is the spectral type and luminosity classification determined from fundamental parameters (method 2). The localization in clusters is indicated in the last column: cl0 for NGC $330\left(0 \mathrm{~h} 56 \min 19 \mathrm{~s}-72^{\circ} 27^{\prime} 52^{\prime \prime}\right)$, cl1 for H86 $170\left(0 \mathrm{~h} 56 \min 21 \mathrm{~s}-72^{\circ} 21^{\prime} 12^{\prime \prime}\right)$, cl2 for [BS95]78 (0h56min04s $\left.-72^{\circ} 20^{\prime} 12^{\prime \prime}\right), \mathrm{cl} 3$ for the association SMC ASS 39 (0h56min6s $\left.-72^{\circ} 18^{\prime} 00^{\prime \prime}\right)$, cl4 for OGLE-SMC109 $\left(0 \mathrm{~h} 57 \mathrm{~min} 29.8 \mathrm{~s}-72^{\circ} 15^{\prime} 51.9^{\prime \prime}\right)$, cl5 for NGC299 (0h53min24.5s $\left.-72^{\circ} 11^{\prime} 49^{\prime \prime}\right)$ corrected coordinates, cl6 for NGC306 (0h54min15s $\left.-72^{\circ} 14^{\prime} 30^{\prime \prime}\right)$, cl7 for H86 145 (0h53min37s $\left.-72^{\circ} 21^{\prime} 00^{\prime \prime}\right)$, cl8 for OGLE-SMC $99\left(0 \mathrm{~h} 54 \min 48.24 \mathrm{~s}-72^{\circ} 27^{\prime} 57.8^{\prime \prime}\right)$; the letter "k" followed by a number corresponds to the star number in Keller et al. (1999), and the "\#" indicates that the star was pre-selected in our catalogue of emission line stars (unpublished). In the last column the spectral classification from the SIMBAD database is also given. The last three lines correspond to emission-line objects which are not Be stars.

\begin{tabular}{|c|c|c|c|c|c|c|c|c|c|c|c|}
\hline Star & $\alpha(2000)$ & $\delta(2000)$ & $V$ & $B-V$ & $S / N$ & $T_{\mathrm{eff}}^{\mathrm{app} .}$ & $\log g_{\text {app. }}$ & $V \sin i_{\text {app. }}$ & RV & $\mathrm{CFP}$ & comm. \\
\hline MHF[S61]47315 & 05449.559 & -722422.35 & - & - & 120 & $30000 \pm 800$ & $3.4 \pm 0.1$ & $370 \pm 10$ & $160 \pm 10$ & $\overline{\mathrm{B} 0 I V}$ & \# \\
\hline MHF[S61]51066 & 05450.936 & -722234.63 & - & - & 130 & $22500 \pm 600$ & $3.3 \pm 0.1$ & $415 \pm 10$ & $130 \pm 10$ & B1III & \# \\
\hline SMC5_000476 & 05323.700 & -722343.80 & 16.36 & -0.15 & 40 & $18500 \pm 1400$ & $4.0 \pm 0.2$ & $309 \pm 30$ & $110 \pm 10$ & $\mathrm{~B} 2 \mathrm{~V}$ & \\
\hline SMC5_000643 & 05544.490 & -722038.00 & 16.30 & -0.12 & 75 & $17500 \pm 700$ & $3.4 \pm 0.2$ & $280 \pm 14$ & $127 \pm 10$ & B3IV & \\
\hline SMC5_002232 & 05605.577 & -723125.91 & 15.60 & -0.09 & 90 & $20500 \pm 600$ & $3.1 \pm 0.1$ & $246 \pm 10$ & $140 \pm 10$ & B2III & \# \\
\hline SMC5 002483 & 05532.170 & -722956.70 & 16.80 & -0.08 & 35 & $19500 \pm 1500$ & $3.8 \pm 0.2$ & $354 \pm 35$ & $151 \pm 10$ & B2IV & $\mathrm{k} 1064$ \\
\hline SMC5 002751 & 05614.260 & -722830.10 & 15.99 & -0.04 & 45 & $17000 \pm 1100$ & $3.2 \pm 0.2$ & $315 \pm 27$ & $143 \pm 10$ & B3III & $\mathrm{cl} 0$ \\
\hline SMC5_002825 & 05441.373 & -722802.42 & 17.43 & -0.11 & 50 & $24500 \pm 1500$ & $4.5 \pm 0.2$ & $277 \pm 22$ & $138 \pm 10$ & B1V & $\mathrm{cl} 8$ \\
\hline SMC5_002957 & 05445.213 & -722713.78 & 15.28 & -0.08 & 135 & $18000 \pm 500$ & $3.0 \pm 0.1$ & $239 \pm 10$ & $137 \pm 10$ & B2III & \# \\
\hline SMC5_002984 & 05548.780 & -722712.70 & 17.40 & -0.09 & 30 & $14500 \pm 1500$ & $3.5 \pm 0.2$ & $317 \pm 48$ & $155 \pm 10$ & B5IV & \\
\hline SMC5_003119 & 05559.900 & -722621.30 & 15.62 & -0.08 & 65 & $17000 \pm 800$ & $3.1 \pm 0.1$ & $262 \pm 21$ & $154 \pm 10$ & B3III & $\mathrm{k} 258$ \\
\hline SMC5_003296 & 05615.964 & -722515.98 & 16.83 & -0.12 & 70 & $20000 \pm 800$ & $3.9 \pm 0.2$ & $181 \pm 10$ & $139 \pm 10$ & B2IV & \#, k916 \\
\hline SMC5_003315 & 05358.250 & -722502.60 & 16.06 & -0.20 & 45 & $19000 \pm 1400$ & $3.3 \pm 0.2$ & $302 \pm 24$ & $171 \pm 10$ & B2III & \\
\hline SMC5_003389 & 05508.973 & -722437.47 & 16.24 & -0.12 & 75 & $18500 \pm 800$ & $3.2 \pm 0.2$ & $306 \pm 15$ & $154 \pm 10$ & B2III & \# \\
\hline SMC5_003537 & 05650.481 & -722340.50 & 16.27 & 0.03 & 50 & $20500 \pm 1300$ & $3.5 \pm 0.2$ & $197 \pm 16$ & $185 \pm 10$ & B2IV & \# \\
\hline SMC5_0 & 0532 & -722207.40 & 17.62 & -0.11 & 25 & $24000 \pm 2400$ & $4.4 \pm 0.2$ & $492 \pm 75$ & $133 \pm 10$ & B1V & EB \\
\hline SMC5_0C & $057 \mathrm{C}$ & -722130.04 & 16.95 & -0.15 & 35 & $23500 \pm 1900$ & $4.2 \pm 0.2$ & $314 \pm 31$ & $150 \pm 10$ & B1V & \# \\
\hline SMC5_0 & $056 ?$ & -722052.80 & 17.53 & -0.18 & 20 & $21500 \pm 2100$ & $4.0 \pm 0.2$ & $498 \pm 75$ & $174 \pm 10$ & $\mathrm{~B} 2 \mathrm{~V}$ & \\
\hline SMC5_004201 & 05612.270 & -721952.90 & 16.33 & -0.08 & 30 & $14000 \pm 1400$ & $2.5 \pm 0.1$ & $483 \pm 70$ & $114 \pm 10$ & B5II-III & \\
\hline SMC5_004509 & 05638.767 & -721812.67 & 16.52 & -0.20 & 60 & $19500 \pm 1000$ & $3.7 \pm 0.2$ & $101 \pm 10$ & $160 \pm 10$ & B2IV & \\
\hline SMC5_004685 & 05559.850 & -721711.40 & 16.36 & -0.07 & 40 & $17500 \pm 1300$ & $3.6 \pm 0.2$ & $245 \pm 25$ & $154 \pm 10$ & B3IV & \\
\hline SMC5_004982 & 05524.880 & -721530.60 & 16.29 & -0.09 & 40 & $20000 \pm 1500$ & $3.7 \pm 0.2$ & $270 \pm 27$ & $138 \pm 10$ & B2IV & \\
\hline SMC5_0 & 05415.010 & -721508.80 & 15.80 & -0.15 & 65 & $20000 \pm 900$ & $3.9 \pm 0.2$ & $226 \pm 13$ & $125 \pm 10$ & B2IV & \\
\hline SMC5_008231 & 05604.698 & -723341.46 & 16.26 & 0.08 & 65 & $21500 \pm 1000$ & $3.2 \pm 0.2$ & $325 \pm 26$ & $161 \pm 10$ & B2III & \# \\
\hline SMC5_009378 & 05708.098 & -723240.68 & 16.51 & -0.01 & 20 & $16500 \pm 1600$ & $3.4 \pm 0.2$ & $349 \pm 50$ & $131 \pm 10$ & B3III & \# \\
\hline SMC5_011371 & 05628.170 & -723036.80 & 16.94 & -0.01 & 30 & $24000 \pm 2400$ & $4.0 \pm 0.2$ & $475 \pm 70$ & $158 \pm 10$ & B1V & \\
\hline SMC5_011991 & 05525.314 & -722956.79 & 16.28 & -0.10 & 60 & $20000 \pm 1000$ & $3.6 \pm 0.2$ & $192 \pm 15$ & $138 \pm 10$ & B2IV & \# \\
\hline SMC5_012717 & 05705.450 & -722916.20 & 16.48 & -0.02 & 20 & $18000 \pm 1800$ & $3.6 \pm 0.2$ & $360 \pm 54$ & $142 \pm 10$ & B2IV & \\
\hline 012767 & 05534.670 & -722913.10 & 16.66 & -0.18 & 35 & $29500 \pm 2800$ & $3.7 \pm 0.2$ & $346 \pm 50$ & $151 \pm 10$ & B0IV & k1054 \\
\hline SMC5_013233 & 05633.898 & -722843.92 & 18.15 & -0.07 & 30 & $14000 \pm 1400$ & $3.9 \pm 0.2$ & $268 \pm 40$ & $107 \pm 10$ & B5IV & 10 \\
\hline SMC5_0 & 056 & -722 & 15.60 & -0.04 & 45 & 17500 & $3.2 \pm 0.2$ & 284 & $144 \pm 10$ & B3III & B2IIIe,cl0, k206 \\
\hline SMC5_0 & 05623.010 & -722753.90 & 15.35 & -0.05 & 75 & $19500 \pm 800$ & $3.4 \pm 0.2$ & $96 \pm 10$ & $159 \pm 10$ & B2III & $\mathrm{cl} 0, \mathrm{k} 215$ \\
\hline SMC5_014114 & 05632.261 & -722750.17 & 15.52 & 0.01 & 90 & $22500 \pm 700$ & $3.2 \pm 0.1$ & $115 \pm 10$ & $153 \pm 10$ & B1III & B2IIIe,cl0, k203 \\
\hline SMC5_014212 & 05432.957 & -722741.85 & 15.42 & -0.08 & 110 & $19500 \pm 500$ & $3.3 \pm 0.1$ & $213 \pm 11$ & $138 \pm 10$ & B2III & \# \\
\hline SMC5 014271 & 05418.131 & -722737.15 & 15.56 & -0.03 & 90 & $30000 \pm 900$ & $3.3 \pm 0.1$ & $499 \pm 17$ & $126 \pm 10$ & B0III & \# \\
\hline SMC5_014637 & 05612.130 & -722716.90 & 15.42 & -0.08 & 60 & $18500 \pm 1000$ & $3.4 \pm 0.2$ & $161 \pm 10$ & $162 \pm 10$ & B2III & $\mathrm{cl} 0, \mathrm{k} 242$ \\
\hline SMC5_014727 & 05618.130 & -722713.50 & 15.65 & -0.05 & 50 & $20000 \pm 1200$ & $3.6 \pm 0.2$ & $315 \pm 16$ & $137 \pm 10$ & B2IV & $\mathrm{cl} 0, \mathrm{k} 228$ \\
\hline SMC5_014864 & 05633.110 & -722704.99 & 16.12 & -0.05 & 90 & $16500 \pm 500$ & $3.4 \pm 0.1$ & $105 \pm 10$ & $120 \pm 10$ & B3III & $\mathrm{cl0}, \#, \mathrm{k} 419$ \\
\hline SMC5_014878 & 05459.339 & -722702.05 & 15.71 & -0.01 & 120 & $21000 \pm 500$ & $3.4 \pm 0.1$ & $404 \pm 20$ & $101 \pm 10$ & B2III & \# \\
\hline SMC5_015429 & 05533.650 & -722629.90 & 17.63 & -0.14 & 25 & $25000 \pm 2500$ & $4.5 \pm 0.2$ & $467 \pm 70$ & $144 \pm 10$ & B1V & k2299 \\
\hline SMC5_015509 & 05624.620 & -722624.70 & 16.98 & -0.04 & 30 & $21500 \pm 2100$ & $3.9 \pm 0.2$ & $476 \pm 70$ & $155 \pm 10$ & B2IV & k857 \\
\hline SMC5_015867 & 05552.279 & -722603.77 & 17.23 & -0.05 & 70 & $20000 \pm 800$ & $3.9 \pm 0.2$ & $207 \pm 10$ & $135 \pm 10$ & B2IV & k991 \\
\hline SMC5_016177 & 05544.521 & -722543.91 & 16.99 & -0.09 & 60 & $24500 \pm 1200$ & $4.2 \pm 0.2$ & $286 \pm 15$ & $123 \pm 10$ & B1V & k1017 \\
\hline
\end{tabular}


Table 3. continued.

\begin{tabular}{|c|c|c|c|c|c|c|c|c|c|c|c|}
\hline Star & $\alpha(2000)$ & $\delta(2000)$ & $V$ & $B-V$ & $S / N$ & $T_{\text {eff }}^{\text {app. }}$ & $\log g_{\text {app. }}$ & $V \sin i_{\text {app. }}$ & $\mathrm{RV}$ & CFP & comm. \\
\hline SMC5_016461 & 05549.619 & -722527.43 & 14.90 & 0.10 & 90 & $17250 \pm 600$ & $2.6 \pm 0.1$ & $330 \pm 14$ & $126 \pm 10$ & B3II/III & EB, k137 \\
\hline SMC5_016477 & 05601.510 & -722 & 18.76 & -0.10 & 20 & $11000 \pm 1100$ & $3.4 \pm 0.2$ & $309 \pm 46$ & $113 \pm 10$ & B9III & \# \\
\hline SMC5_016486 & 05446.418 & -722522.76 & 15.58 & -0.04 & 135 & $25000 \pm 700$ & $3.3 \pm 0.1$ & $496 \pm 20$ & $140 \pm 10$ & B1III & \# \\
\hline SMC5_016523 & 05530.790 & -722520.30 & 15.70 & -0.06 & 60 & $20000 \pm 1000$ & $2 \pm 0.2$ & $430 \pm 22$ & $130 \pm 10$ & B2III & k278 \\
\hline SMC5_016544 & 05629.100 & -722521.50 & 16.84 & -0.01 & 55 & $21000 \pm 1000$ & $3.6 \pm 0.2$ & $344 \pm 17$ & $107 \pm 10$ & B2IV & \#, k837 \\
\hline SMC5_016824 & 05344.010 & -722456.30 & 15.06 & -0.12 & 70 & $18500 \pm 800$ & $3.4 \pm 0.2$ & $188 \pm 10$ & $161 \pm 10$ & B2IV & \\
\hline SMC5_017596 & 05633.330 & -722419.80 & 17.16 & -0.01 & 35 & $19000 \pm 1900$ & $3.8 \pm 0.2$ & $202 \pm 20$ & $143 \pm 10$ & B2IV & $\mathrm{k} 818$ \\
\hline SMC5_018501 & 05614.450 & -722323.60 & 15.20 & -0.08 & 55 & $23500 \pm 1200$ & $3.7 \pm 0.2$ & $357 \pm 18$ & $183 \pm 10$ & B1IV & k128 \\
\hline SMC5_020211 & 05606.798 & -722135.34 & 16.86 & -0.17 & 50 & $21500 \pm 1100$ & $3.5 \pm 0.2$ & $337 \pm 17$ & $154 \pm 10$ & B2IV & \\
\hline SMC5_021152 & 05312.660 & -722029.50 & 15.30 & -0.18 & 60 & $18000 \pm 900$ & $3.0 \pm 0.2$ & $199 \pm 10$ & $144 \pm 10$ & B2III & \\
\hline SMC5_021886 & 05548.566 & -721946.88 & 17.50 & -0.10 & 50 & $22000 \pm 1100$ & $3.7 \pm 0.2$ & $409 \pm 21$ & $93 \pm 10$ & B2IV & \\
\hline SMC5_022295 & 05514.500 & -721918.60 & 15.92 & -0.10 & 40 & $18500 \pm 1000$ & $3.2 \pm 0.2$ & $346 \pm 27$ & $121 \pm 10$ & B2III & \\
\hline SMC5_022628 & 05337.080 & -721850.60 & 15.86 & -0.09 & 50 & $22500 \pm 1100$ & $3.5 \pm 0.2$ & $457 \pm 24$ & $113 \pm 10$ & B1III-IV & \\
\hline SMC5_022842 & 05549.880 & -721842.10 & 17.77 & -0.18 & 25 & $21500 \pm 2100$ & $3.9 \pm 0.2$ & $493 \pm 70$ & $95 \pm 10$ & B2IV & \\
\hline SMC5_C & 0562 & -721720.79 & 17.36 & -0.12 & 75 & $20000 \pm 800$ & $3.6 \pm 0.2$ & $349 \pm 17$ & $133 \pm 10$ & B2IV & \\
\hline SMC5_C & 05 & -721 & 17.72 & -0.12 & 25 & $14000 \pm 1400$ & 0.2 & 50 & 134 & B5IV & \\
\hline SMC5_ & 05 & -721 & 17.97 & -0.09 & 25 & 16500 & & & 152 & B3V & \\
\hline SMC5_ & 05 & -721 & 16.87 & -0.16 & 40 & $19500 \pm 1000$ & 0.2 & $=17$ & $150 \pm 10$ & B2III & \\
\hline 816 & 05 & -721 & 15.62 & -0.09 & 60 & 900 & 0.2 & 11 & $136 \pm 10$ & B2III & \\
\hline 829 & 056 & -721 & 16.23 & -0.11 & 45 & 1100 & 0.2 & $=17$ & $151 \pm 10$ & B2III-IV & \\
\hline SMC5_026182 & 054 & -721 & 17.98 & -0.13 & 24 & 1500 & 0.2 & \pm 50 & $153 \pm 10$ & B3IV & cl6 \\
\hline SMC5_026689 & 0540 & -721403.90 & 17.52 & -0.09 & 25 & $19500 \pm 1900$ & $4.2 \pm 0.2$ & $397 \pm 55$ & $144 \pm 10$ & $\mathrm{~B} 2 \mathrm{~V}$ & \\
\hline SMC5_0 & .930 & -721204.60 & 16.61 & -0.05 & 35 & $18500 \pm 1800$ & $3.3 \pm 0.2$ & $499 \pm 75$ & $136 \pm 10$ & B2III & \\
\hline 967 & 0554 & -722944.70 & 16.36 & -0.14 & 40 & $18500 \pm 1400$ & $3.2 \pm 0.2$ & \pm 20 & $150 \pm 10$ & B2III & k528 \\
\hline SMC5 & 05 & -722 & 15.50 & -0.02 & 110 & 00 & & $\leqslant 10$ & & B2III & \# \\
\hline SMC5_( & 0562 & -722809.40 & 15.84 & -0.09 & 95 & 2000 & $3.5 \pm 0.1$ & $286 \pm 10$ & $148 \pm 10$ & B2III & $\mathrm{cl} 0, \#, \mathrm{k} 211$ \\
\hline 7158 & 05 & -722754.60 & 17.23 & -0.08 & 30 & 23000 & $3.7 \pm 0.2$ & \pm 60 & $151 \pm 10$ & B1IV & k1041 \\
\hline SMC5_ & 054 & -722752.50 & 16.02 & -0.08 & 35 & 20500 & 0.2 & 50 & 10 & B2III & $\mathrm{cl} 8$ \\
\hline 007 & 053 & -721955.42 & 17.27 & -0.18 & 60 & 1650 & & 13 & $152 \pm 10$ & B3V & \\
\hline SM & 05 & -721 & 17.72 & -0.13 & 30 & 12500 & & 40 & 131 & B7IV & \\
\hline SMC & 05 & -721 & 16.54 & -0.10 & 80 & 700 & 0.1 & 18 & 133 & B1IV & \# \\
\hline SM & 05 & -723 & 16.37 & -0.03 & 70 & 00 & & 40 & 10 & B1III & \# \\
\hline 413 & 05 & -722 & 15.84 & -0.04 & 35 & 400 & .2 & 41 & 10 & B2IV & k27 \\
\hline 117 & 05 & -722 & 16.34 & -0.09 & 35 & 2000 & 0.2 & 50 & 10 & B2IV & $\mathrm{cl} 0, \mathrm{k} 471$ \\
\hline SMC5_0 & 0561 & -722801.57 & 15.96 & -0.07 & 95 & $19500 \pm 600$ & 0.1 & 10 & $140 \pm 10$ & B2III & $\mathrm{cl} 0, \mathrm{k} 222$ \\
\hline SMC5_044898 & $056 \mathrm{C}$ & -722743.74 & 16.85 & -0.03 & 70 & $20000 \pm$ & $3.5 \pm 0.2$ & 20 & $145 \pm 10$ & B2III-IV & $\mathrm{cl} 0, \#, \mathrm{k} 480$ \\
\hline SMC5_0 & 0542 & -722707.62 & 15.53 & -0.11 & 120 & $19000 \pm 500$ & $3.2 \pm 0.1$ & \pm 10 & $158 \pm 10$ & B2III & \# \\
\hline SMC5_0 & 0554 & -722641.70 & 16.58 & -0.04 & 45 & $18500 \pm 1400$ & $3.4 \pm 0.2$ & \pm 18 & $118 \pm 10$ & B2III-IV & k529 \\
\hline SMC5_0 & 0532 & -722541.40 & 16.47 & -0.05 & 30 & \pm 2000 & $3.6 \pm 0.2$ & $330 \pm 50$ & $95 \pm 10$ & B2IV & \\
\hline SMC5_046462 & 05622.590 & -722547.20 & 16.76 & -0.03 & 40 & $21000 \pm 1500$ & $3.7 \pm 0.2$ & $348 \pm 27$ & $120 \pm 10$ & B2IV & k874 \\
\hline SMC & 055 & -722358.20 & 16.04 & -0.08 & 40 & $0 \pm 900$ & \pm 0.2 & 10 & $159 \pm 10$ & B7II-III & k522 \\
\hline SMC5_048045 & 05556.320 & -722333.30 & 16.59 & -0.11 & 45 & $18500 \pm 1400$ & $3.3 \pm 0.2$ & $331 \pm 21$ & $145 \pm 10$ & B2III & k509 \\
\hline SMC5_048047 & 05730.577 & -722333.23 & 15.90 & -0.12 & 80 & $19500 \pm 600$ & $3.4 \pm 0.1$ & $224 \pm 11$ & $117 \pm 10$ & B2IV & \# \\
\hline SMC5_0 & 0535 & $\begin{array}{ll}-72 & 2309.20\end{array}$ & 15.87 & -0.17 & 50 & $19500 \pm 1200$ & $3.8 \pm 0.2$ & $253 \pm 14$ & $133 \pm 10$ & B2IV & \\
\hline 651 & 056 & -722117.05 & 17.86 & -0.21 & 42 & $14500 \pm 1100$ & $4.1 \pm 0.2$ & \pm 19 & $86 \pm 10$ & B5V & cl1 \\
\hline 746 & 05 & -722106.30 & 16.49 & -0.16 & 35 & $19500 \pm 1900$ & 0.2 & \pm 60 & $118 \pm 10$ & B2III-IV & \\
\hline & 05 & -722 & 16.71 & -0.07 & 60 & 00 & & 24 & & B1IV & \# \\
\hline 996 & 05 & -722042.30 & 15.88 & -0.12 & 45 & 19500 & 3.2 & $=30$ & 155 & B2III & \\
\hline 315 & 0540 & -721850.70 & 17.96 & -0.16 & 20 & 1350 & 4.0 & 21 & 113 & B5V & \\
\hline 688 & 05547.140 & -721634.00 & 15.49 & -0.14 & 70 & $18500 \pm 800$ & $3.3 \pm 0.2$ & $279 \pm 14$ & $129 \pm 10$ & B2III & \\
\hline 267 & 055 & -721539.90 & 17.04 & -0.06 & 35 & $19000 \pm 1900$ & $3.8 \pm 0.2$ & $449 \pm 65$ & $140 \pm 10$ & B2IV & \\
\hline 756 & 0541 & -721448.00 & 16.53 & -0.08 & 75 & $19500 \pm 800$ & 0.2 & \pm 10 & $139 \pm 10$ & B2IV & cl6 \\
\hline 592 & 05322.720 & -721155.70 & 16.68 & -0.11 & 40 & $17000 \pm 1200$ & $3.6 \pm 0.2$ & $118 \pm 10$ & $162 \pm 10$ & B3IV & $\mathrm{cl} 5$ \\
\hline SMC5_061950 & 05629.960 & -721441.70 & 17.67 & -0.14 & 30 & $13500 \pm 1300$ & $3.5 \pm 0.2$ & $302 \pm 45$ & $154 \pm 10$ & B5III & \\
\hline SMC5_064327 & 05614.900 & -722847.50 & 15.41 & -0.03 & 80 & $17000 \pm 500$ & $2.8 \pm 0.1$ & $283 \pm 14$ & $147 \pm 10$ & B3II-III & $\mathrm{cl} 0, \mathrm{k} 238$ \\
\hline SMC5_064576 & 05446.290 & -722805.00 & 17.38 & -0.10 & 30 & $20500 \pm 1000$ & $4.2 \pm 0.2$ & $308 \pm 45$ & $156 \pm 10$ & $\mathrm{~B} 2 \mathrm{~V}$ & $\mathrm{cl} 8$ \\
\hline SMC5_064745 & 05428.845 & -722738.11 & 15.691 & -0.07 & 55 & $30000 \pm 1600$ & $3.4 \pm 0.2$ & $447 \pm 36$ & $161 \pm 10$ & B0IV & \# \\
\hline SMC5_064832 & 05454.577 & -722723.64 & 16.79 & 0.07 & 60 & $15500 \pm 800$ & $3.4 \pm 0.2$ & $300 \pm 15$ & $125 \pm 10$ & B3III & \\
\hline SMC5_065055 & 05355.340 & -722645.30 & 14.72 & -0.03 & 70 & $24000 \pm 900$ & $3.2 \pm 0.2$ & $420 \pm 21$ & $140 \pm 10$ & B1III & \\
\hline
\end{tabular}


Table 3. continued.

\begin{tabular}{|c|c|c|c|c|c|c|c|c|c|c|c|}
\hline Star & $\alpha(2000)$ & $\delta(2000)$ & $V$ & $B-V$ & $S / N$ & $T_{\text {eff }}^{\text {app. }}$ & $\log g_{\text {app. }}$ & $V \sin i_{\text {app. }}$ & $\mathrm{RV}$ & $\mathrm{CFP}$ & comm. \\
\hline 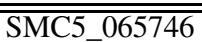 & $\overline{05501.887}$ & $=-722445.57$ & $\overline{17.42}$ & -0.07 & 50 & $24000 \pm 1400$ & $\overline{4.3 \pm 0.2}$ & $362 \pm 19$ & $\overline{105 \pm 10}$ & B1V & \\
\hline SMC5_066754 & 05521.820 & -722133.70 & 16.09 & -0.16 & 40 & $19500 \pm 1400$ & $3.6 \pm 0.2$ & $165 \pm 10$ & $152 \pm 10$ & B2IV & \\
\hline SMC5_067333 & 05651.700 & -721945.20 & 16.21 & -0.03 & 35 & $19500 \pm 1900$ & $3.8 \pm 0.2$ & $271 \pm 23$ & $140 \pm 10$ & B2IV & \\
\hline SMC5_073581 & 05626.600 & -722623.00 & 16.22 & -0.12 & 40 & $17000 \pm 1200$ & $3.4 \pm 0.2$ & $227 \pm 15$ & $135 \pm 10$ & B3III & \\
\hline SMC5_073594 & 05321.410 & -722608.90 & 16.13 & -0.05 & 40 & $17500 \pm 1300$ & $3.5 \pm 0.2$ & $192 \pm 12$ & $166 \pm 10$ & B2-3III-IV & \\
\hline SMC5_074402 & 05304.530 & -722049.30 & 15.83 & -0.08 & 50 & $18000 \pm 1100$ & $3.1 \pm 0.2$ & $393 \pm 20$ & $147 \pm 10$ & B2III & \\
\hline SMC5_074471 & 05326.610 & -722018.60 & 14.97 & -0.18 & 70 & $19000 \pm 800$ & $3.2 \pm 0.2$ & $185 \pm 10$ & $158 \pm 10$ & B2III & \\
\hline SMC5_075061 & 05630.580 & -721616.20 & 17.58 & -0.10 & 30 & $16000 \pm 1600$ & $3.8 \pm 0.2$ & $122 \pm 20$ & $136 \pm 10$ & B3IV & \\
\hline SMC5_075360 & 05405.990 & -721351.60 & 15.78 & -0.12 & 65 & $19000 \pm 900$ & $3.6 \pm 0.2$ & $234 \pm 10$ & $181 \pm 10$ & B2IV & \\
\hline SMC5_078338 & 05625.450 & -722707.00 & 15.49 & -0.05 & 45 & $19000 \pm 1400$ & $3.4 \pm 0.2$ & $73 \pm 10$ & $155 \pm 10$ & B2III & B2IIIe, c10, k213 \\
\hline SMC5_078440 & 05650.560 & -721507.30 & 15.63 & -0.04 & 60 & $18000 \pm 900$ & $2.8 \pm 0.2$ & $348 \pm 17$ & $160 \pm 10$ & B2II-III & \\
\hline SMC5_078928 & 05709.690 & -722657.50 & 17.48 & -0.23 & 25 & $19000 \pm 1900$ & $3.7 \pm 0.2$ & $139 \pm 21$ & $165 \pm 10$ & B2IV & \\
\hline SMC5_080910 & 05424.272 & -721349.41 & 16.74 & -0.16 & 65 & $19500 \pm 1000$ & $3.7 \pm 0.2$ & $316 \pm 15$ & $146 \pm 10$ & B2IV & \\
\hline SMC5_081260 & 05413.120 & -721435.60 & 17.77 & -0.11 & 40 & $15500 \pm 1100$ & $4.2 \pm 0.2$ & $227 \pm 15$ & $139 \pm 10$ & B3V & cl6 \\
\hline SMC5_082042 & 05618.260 & -721746.80 & 16.39 & -0.14 & 40 & $17500 \pm 1300$ & $2.8 \pm 0.2$ & $405 \pm 26$ & $151 \pm 10$ & B3III & \\
\hline SMC5_082202 & 05730.310 & -721558.40 & 15.76 & -0.24 & 75 & $15000 \pm 600$ & $3.4 \pm 0.2$ & $265 \pm 13$ & $139 \pm 10$ & B5III & cl4 \\
\hline SMC5_082543 & 05654.005 & -722850.18 & 17.05 & 0.01 & 55 & $22500 \pm 1200$ & $3.9 \pm 0.2$ & $360 \pm 18$ & $143 \pm 10$ & B1IV & k754 \\
\hline SMC5_082819 & 05607.190 & -722813.70 & 13.46 & 0.04 & 130 & $20000 \pm 500$ & $2.8 \pm 0.1$ & $318 \pm 10$ & $133 \pm 10$ & B2III & $\mathrm{cl0}, \#$ \\
\hline SMC5_082941 & 05353.660 & -722201.40 & 15.75 & -0.12 & 50 & $17500 \pm 900$ & $3.1 \pm 0.2$ & $323 \pm 16$ & $151 \pm 10$ & B3III & \\
\hline SMC5_083491 & 05319.930 & $\begin{array}{l}-722229.20 \\
\end{array}$ & 15.95 & -0.07 & 45 & $17500 \pm 900$ & $2.9 \pm 0.2$ & $433 \pm 28$ & $133 \pm 10$ & B3III & \\
\hline SMC5_085503 & 05447.457 & -722758.77 & 16.88 & -0.03 & 80 & $15000 \pm 500$ & $3.0 \pm 0.2$ & $275 \pm 14$ & $129 \pm 10$ & B5III & $\mathrm{cl} 8$ \\
\hline SMC5_086200 & 05532.480 & -722752.00 & 17.29 & -0.13 & 30 & $19000 \pm 1900$ & $3.7 \pm 0.2$ & $370 \pm 55$ & $125 \pm 10$ & B2IV & k1062 \\
\hline SMC5_086251 & 05535.310 & -721511.70 & 16.86 & -0.15 & 40 & $19500 \pm 1500$ & $3.8 \pm 0.2$ & $298 \pm 19$ & $145 \pm 10$ & B2IV & \\
\hline SMC5_086581 & 05555.498 & -722658.34 & 17.53 & -0.06 & 55 & $15000 \pm 900$ & $4.0 \pm 0.2$ & $310 \pm 15$ & $114 \pm 10$ & B3IV-V & k2118 \\
\hline SMC5_086890 & 05616.439 & -722756.38 & 16.88 & 0.04 & 70 & $24000 \pm 900$ & $3.9 \pm 0.2$ & $383 \pm 19$ & $130 \pm 10$ & B1IV & $\mathrm{cl} 0, \mathrm{k} 462$ \\
\hline SMC5_086983 & 05620.410 & -722806.40 & 16.24 & -0.06 & 40 & $21500 \pm 1600$ & $3.7 \pm 0.2$ & $389 \pm 25$ & $140 \pm 10$ & B2IV & $\mathrm{cl} 0, \mathrm{k} 441$ \\
\hline SMC5_087004 & 05621.394 & -722727.89 & 17.18 & -0.08 & 60 & $23500 \pm 1200$ & $4.1 \pm 0.2$ & $384 \pm 19$ & $134 \pm 10$ & B1V & $\mathrm{cl} 0, \mathrm{k} 882$ \\
\hline SMC5_090914 & 05620.250 & -722728.70 & 16.03 & -0.06 & 45 & $16500 \pm 1200$ & $3.3 \pm 0.2$ & $310 \pm 20$ & $144 \pm 10$ & B3III & $\mathrm{cl} 0, \mathrm{k} 442$ \\
\hline SMC5_190576 & 05644.310 & -722906.30 & 14.56 & -0.12 & 95 & $30000 \pm 900$ & $3.2 \pm 0.1$ & $393 \pm 14$ & $148 \pm 10$ & B0III & \\
\hline SMC5_002807 & 05609.420 & -722809.30 & 14.62 & 1.08 & 15 & & & & & $\mathrm{cool} \mathrm{Sg/EB}$ & Sg K, cl0, k44 \\
\hline SMC5_037102 & 05606.450 & -722827.70 & 17.32 & 0.46 & 20 & & & & & $\mathrm{HB}[\mathrm{e}]$ & $\mathrm{HBe}, \mathrm{AGB}, \mathrm{cl} 0, \mathrm{k} 485$ \\
\hline SMC5_081994 & 05630.750 & -722702.00 & 17.32 & -0.18 & 10 & & & & & PN & $\mathrm{PN}, \mathrm{cl0}, \mathrm{k} 4154$ \\
\hline
\end{tabular}


C. Martayan et al.: B and Be stars in the SMC. II., Online Material p 13

Table 4. Apparent parameters $\log \left(L / L_{\odot}\right), M / M_{\odot}$, and $R / R_{\odot}$ interpolated or calculated for Be stars in the SMC from HR diagrams published in Schaller et al. (1992) for $Z=0.001$.

\begin{tabular}{|c|c|c|c|c|}
\hline$\overline{\text { Star }}$ & $\log \left(L / L_{\odot}\right)$ & $\bar{M} / M_{\odot}$ & $R / R_{\odot}$ & age Myears \\
\hline MHF[S9]47315 & $5.3 \pm 0.4$ & $23.9 \pm 1.5$ & $16.0 \pm 1.5$ & $8 \pm 1$ \\
\hline MHF[S9]51066 & $4.6 \pm 0.4$ & $13.1 \pm 1.0$ & $14.1 \pm 1.5$ & $16 \pm 3$ \\
\hline SMC5_000476 & $3.2 \pm 0.4$ & $5.2 \pm 0.5$ & $3.8 \pm 0.5$ & 6 \\
\hline SMC5_000643 & $3.8 \pm 0.4$ & $7.2 \pm 0.5$ & $8.7 \pm 1.0$ & $44 \pm 3$ \\
\hline SMC5_002232 & $4.7 \pm 0.4$ & $14.0 \pm 1.0$ & $18.0 \pm 1.5$ & $15 \pm 3$ \\
\hline SMC5_002483 & $3.5 \pm 0.4$ & $6.3 \pm 0.5$ & $5.2 \pm 1.0$ & $61 \pm 6$ \\
\hline SMC5_002751 & $4.1 \pm 0.4$ & $8.8 \pm 0.5$ & $13.2 \pm 1.5$ & $30 \pm 3$ \\
\hline SMC5_002825 & $3.3 \pm 0.4$ & $7.0 \pm 0.5$ & $2.5 \pm 0.5$ & $7 \pm 1$ \\
\hline SMC5_002957 & $4.5 \pm 0.4$ & $12.0 \pm 1.0$ & $18.6 \pm 1.5$ & $18 \pm 3$ \\
\hline SMC5_002984 & $3.3 \pm 0.4$ & $4.9 \pm 0.5$ & $6.8 \pm 1.0$ & $96 \pm 6$ \\
\hline SMC5_003119 & $4.2 \pm 0.4$ & $9.6 \pm 0.5$ & $15.0 \pm 1.5$ & $27 \pm 3$ \\
\hline SMC5_003296 & $3.5 \pm 0.4$ & $6.1 \pm 0.5$ & $4.7 \pm 0.5$ & $63 \pm 6$ \\
\hline SMC5_003315 & $4.1 \pm 0.4$ & $8.7 \pm 0.5$ & $11.3 \pm 1.5$ & $31 \pm 3$ \\
\hline SMC5_003389 & $4.2 \pm 0.4$ & $9.7 \pm 1.0$ & $13.2 \pm 1.5$ & $26 \pm 3$ \\
\hline SMC5_003537 & $4.1 \pm 0.4$ & $8.8 \pm 0.5$ & $8.6 \pm 1.0$ & $30 \pm 3$ \\
\hline SMC5_003789 & $3.4 \pm 0.4$ & $7.0 \pm 0.5$ & $2.8 \pm 0.5$ & $18 \pm 3$ \\
\hline SMC5_003919 & $3.6 \pm 0.4$ & $7.4 \pm 0.5$ & $3.7 \pm 0.5$ & $30 \pm 3$ \\
\hline SMC5_004026 & $3.6 \pm 0.4$ & $7.0 \pm 0.5$ & $4.4 \pm 0.5$ & $40 \pm 3$ \\
\hline SMC5_004201 & $4.5 \pm 0.4$ & $12.0 \pm 1.0$ & $35.8 \pm 2.5$ & $18 \pm 3$ \\
\hline SMC5_004509 & $3.7 \pm 0.4$ & $6.9 \pm 0.5$ & $6.3 \pm 1.0$ & $48 \pm 3$ \\
\hline SMC5_004685 & $3.5 \pm 0.4$ & $6.1 \pm 0.5$ & $6.6 \pm 1.0$ & $4 \pm 6$ \\
\hline SMC5_004982 & $3.7 \pm 0.4$ & $6.8 \pm 0.5$ & $5.9 \pm 1.0$ & $49 \pm 3$ \\
\hline SMC5_005045 & $3.5 \pm 0.4$ & $6.0 \pm 0.5$ & $4.6 \pm 0.5$ & $64 \pm 6$ \\
\hline SMC5_008231 & $4.6 \pm 0.4$ & $12.9 \pm 1.0$ & $15.1 \pm 1.5$ & $17 \pm 3$ \\
\hline SMC5_009378 & $3.7 \pm 0.4$ & $6.7 \pm 0.5$ & $9.2 \pm 1.0$ & $52 \pm 6$ \\
\hline SMC5_011371 & $3.8 \pm 0.4$ & $8.2 \pm 0.5$ & $4.5 \pm 0.5$ & $29 \pm 3$ \\
\hline SMC5_0 & $3.8 \pm 0.4$ & $7.6 \pm 0.5$ & $7.2 \pm 1.0$ & $41 \pm 3$ \\
\hline SMC5_012717 & $3.7 \pm 0.4$ & $6.6 \pm 0.5$ & $6.9 \pm 1.0$ & $55 \pm 6$ \\
\hline SMC5_01 & $4.8 \pm 0.4$ & $16.8 \pm 1.0$ & $9.9 \pm 1.0$ & $12 \pm 3$ \\
\hline SMC5_0 & $2.6 \pm 0.4$ & $3.3 \pm 0.5$ & $3.3 \pm 0.5$ & $235 \pm 10$ \\
\hline SMC5_0 & $4.2 \pm 0.4$ & $9.4 \pm 0.5$ & $13.9 \pm 1.5$ & 3 \\
\hline SMC5_0 & $4.2 \pm 0.4$ & $9.4 \pm 0.5$ & $11.2 \pm 1.5$ & $27 \pm 3$ \\
\hline SMC5_0 & $4.7 \pm 0.4$ & $14.7 \pm 1.0$ & $15.9 \pm 1.5$ & $14 \pm 3$ \\
\hline SMC5_014212 & $4.3 \pm 0.4$ & $10.2 \pm 1.0$ & $13.0 \pm 1.5$ & $25 \pm 3$ \\
\hline SMC5_014271 & $5.4 \pm 0.4$ & $26.6 \pm 2.0$ & $18.2 \pm 1.5$ & $7 \pm 1$ \\
\hline SMC5_014637 & $4.0 \pm 0.4$ & $8.5 \pm 0.5$ & $10.5 \pm 1.5$ & $33 \pm 3$ \\
\hline SMC5_014727 & $3.8 \pm 0.4$ & $7.4 \pm 0.5$ & $6.9 \pm 1.0$ & $42 \pm 3$ \\
\hline SMC5_014864 & $3.6 \pm 0.4$ & $6.4 \pm 0.5$ & $8.2 \pm 1.0$ & $59 \pm 6$ \\
\hline SMC5_014878 & $4.4 \pm 0.4$ & $11.0 \pm 1.0$ & $12.3 \pm 1.5$ & $22 \pm 3$ \\
\hline SMC5_015429 & $3.3 \pm 0.4$ & $7.1 \pm 0.5$ & $2.5 \pm 0.5$ & $4 \pm 1$ \\
\hline SMC5_015509 & $3.6 \pm 0.4$ & $7.0 \pm 0.5$ & $4.8 \pm 0.5$ & $42 \pm 3$ \\
\hline SMC5_0 & $3.5 \pm 0.4$ & $6.2 \pm 0.5$ & $4.7 \pm 0.5$ & $61 \pm 6$ \\
\hline SMC5_016177 & $3.6 \pm 0.4$ & $7.7 \pm 0.5$ & $3.6 \pm 0.5$ & $27 \pm 3$ \\
\hline SMC5_016461 & $5.0 \pm 0.4$ & $16.8 \pm 1.0$ & $36.8 \pm 2.0$ & $12 \pm 3$ \\
\hline SMC & $2.7 \pm 0.4$ & $3.4 \pm 0.5$ & $6.4 \pm 1.0$ & $232 \pm 10$ \\
\hline SMC5_0 & $5.0 \pm 0.4$ & $18.0 \pm 1.0$ & $16.7 \pm 1.5$ & $11 \pm 3$ \\
\hline SMC5_0 & $4.5 \pm 0.4$ & $12.3 \pm 1.0$ & $16.0 \pm 1.5$ & $18 \pm 3$ \\
\hline SMC5_0 & $4.0 \pm 0.4$ & $8.6 \pm 0.5$ & $7.8 \pm 1.0$ & $32 \pm 3$ \\
\hline SMC5_016824 & $3.9 \pm 0.4$ & $7.6 \pm 0.5$ & $8.8 \pm 1.0$ & $40 \pm 3$ \\
\hline SMC5_017596 & $3.5 \pm 0.4$ & $6.0 \pm 0.5$ & $5.1 \pm 1.0$ & $66 \pm 6$ \\
\hline SMC5_018501 & $4.2 \pm 0.4$ & $10.0 \pm 1.0$ & $7.7 \pm 1.0$ & $25 \pm 3$ \\
\hline SMC5_020211 & $4.2 \pm 0.4$ & $9.7 \pm 1.0$ & $9.2 \pm 1.0$ & $26 \pm 3$ \\
\hline SMC5_021152 & $4.5 \pm 0.4$ & $11.6 \pm 1.0$ & $18.1 \pm 1.5$ & $20 \pm 3$ \\
\hline SMC5_021886 & $4.0 \pm 0.4$ & $8.7 \pm 0.5$ & $7.2 \pm 1.0$ & $32 \pm 3$ \\
\hline SMC5_022295 & $4.3 \pm 0.4$ & $10.8 \pm 1.0$ & $15.0 \pm 1.5$ & $22 \pm 3$ \\
\hline SMC5_022628 & $4.3 \pm 0.4$ & $10.8 \pm 1.0$ & $10.4 \pm 1.5$ & $23 \pm 3$ \\
\hline SMC5_022842 & $3.7 \pm 0.4$ & $7.0 \pm 0.5$ & $4.9 \pm 0.5$ & $43 \pm 3$ \\
\hline SMC5_023931 & $3.9 \pm 0.4$ & $8.0 \pm 0.5$ & $7.7 \pm 1.0$ & $37 \pm 3$ \\
\hline
\end{tabular}


Table 4. continued.

\begin{tabular}{|c|c|c|c|c|}
\hline$\overline{\text { Star }}$ & $\overline{\log \left(L / L_{\odot}\right)}$ & $\bar{M} / M_{\odot}$ & $\overline{R R / R_{\odot}}$ & age Myears \\
\hline SMC5_025052 & $2.9 \pm 0.4$ & $3.8 \pm 0.5$ & $4.7 \pm 0.5$ & $178 \pm 10$ \\
\hline SMC5_025589 & $2.8 \pm 0.4$ & $4.0 \pm 0.5$ & $3.2 \pm 0.5$ & $129 \pm 3$ \\
\hline SMC5_025718 & $4.3 \pm 0.4$ & $10.5 \pm 1.0$ & $13.0 \pm 1.5$ & $24 \pm 3$ \\
\hline SMC5_025816 & $4.2 \pm 0.4$ & $9.3 \pm 0.5$ & $13.2 \pm 1.5$ & $28 \pm 3$ \\
\hline SMC5_025829 & $3.9 \pm 0.4$ & $8.0 \pm 0.5$ & $9.0 \pm 1.0$ & $37 \pm 3$ \\
\hline SMC5_026182 & $2.7 \pm 0.4$ & $3.6 \pm 0.5$ & $3.4 \pm 0.5$ & $195 \pm 10$ \\
\hline SMC5_026689 & $3.1 \pm 0.4$ & $5.2 \pm 0.5$ & $3.1 \pm 0.5$ & $65 \pm 6$ \\
\hline SMC5_028368 & $4.2 \pm 0.4$ & $9.4 \pm 0.5$ & $12.6 \pm 1.5$ & $28 \pm 3$ \\
\hline SMC5_036967 & $4.3 \pm 0.4$ & $10.7 \pm 1.0$ & $14.9 \pm 1.5$ & $23 \pm 3$ \\
\hline SMC5_037013 & $4.5 \pm 0.4$ & $12.2 \pm 1.0$ & $16.4 \pm 1.5$ & $18 \pm 3$ \\
\hline SMC5_037137 & $4.1 \pm 0.4$ & $9.1 \pm 0.5$ & $9.4 \pm 1.0$ & $29 \pm 3$ \\
\hline SMC5_037158 & $4.1 \pm 0.4$ & $9.2 \pm 0.5$ & $6.9 \pm 1.0$ & $28 \pm 3$ \\
\hline SMC5_037162 & $4.1 \pm 0.4$ & $9.2 \pm 0.5$ & $9.2 \pm 1.0$ & $28 \pm 3$ \\
\hline SMC5_038007 & $2.9 \pm 0.4$ & $4.3 \pm 0.5$ & $3.4 \pm 0.5$ & $116 \pm 6$ \\
\hline SMC5_038312 & $2.8 \pm 0.4$ & $3.5 \pm 0.5$ & $5.4 \pm 1.0$ & $212 \pm 10$ \\
\hline SMC5_038363 & $4.1 \pm 0.4$ & $9.3 \pm 0.5$ & $7.2 \pm 1.0$ & $28 \pm 3$ \\
\hline SMC5_041410 & $4.5 \pm 0.4$ & $12.0 \pm 1.0$ & $13.2 \pm 1.5$ & $18 \pm 3$ \\
\hline SMC5_043413 & $3.8 \pm 0.4$ & $7.1 \pm 0.5$ & $6.7 \pm 1.0$ & $45 \pm 3$ \\
\hline SMC5_044117 & $3.6 \pm 0.4$ & $6.5 \pm 0.5$ & $5.4 \pm 1.0$ & $56 \pm 6$ \\
\hline SMC5_044693 & $4.2 \pm 0.4$ & $9.7 \pm 1.0$ & $11.6 \pm 1.5$ & $26 \pm 3$ \\
\hline SMC5_044898 & $4.0 \pm 0.4$ & $8.6 \pm 0.5$ & $9.0 \pm 1.0$ & $32 \pm 3$ \\
\hline SMC5_045353 & $4.3 \pm 0.4$ & $10.8 \pm 1.0$ & $14.7 \pm 1.5$ & $22 \pm 3$ \\
\hline SMC5_045747 & $3.9 \pm 0.4$ & $7.8 \pm 0.5$ & $8.9 \pm 1.0$ & $39 \pm 3$ \\
\hline SMC5_046388 & $3.9 \pm 0.4$ & $8.0 \pm 0.5$ & $7.6 \pm 1.0$ & $37 \pm 3$ \\
\hline SMC5_046462 & $3.9 \pm 0.4$ & $7.9 \pm 0.5$ & $6.8 \pm 1.0$ & $38 \pm 3$ \\
\hline SMC5_047763 & $4.2 \pm 0.4$ & $9.0 \pm 0.5$ & $27.0 \pm 2.0$ & $30 \pm 3$ \\
\hline SMC5_048045 & $4.2 \pm 0.4$ & $9.4 \pm 0.5$ & $12.5 \pm 1.5$ & $28 \pm 3$ \\
\hline SMC5_048047 & $4.1 \pm 0.4$ & $8.8 \pm 0.5$ & $9.6 \pm 1.0$ & $31 \pm 3$ \\
\hline SMC5_048289 & $3.5 \pm 0.4$ & $6.3 \pm 0.5$ & $5.2 \pm 1.0$ & $61 \pm 6$ \\
\hline SMC5_049651 & $2.4 \pm 0.4$ & $3.1 \pm 0.5$ & $2.6 \pm 0.5$ & $240 \pm 10$ \\
\hline SMC5_049746 & $4.0 \pm 0.4$ & $8.3 \pm 0.5$ & $8.8 \pm 1.0$ & $35 \pm 3$ \\
\hline SMC5_049780 & $4.3 \pm 0.4$ & $10.5 \pm 1.0$ & $8.9 \pm 1.0$ & $24 \pm 3$ \\
\hline SMC5_049996 & $4.4 \pm 0.4$ & $11.1 \pm 1.0$ & $14.4 \pm 1.5$ & $21 \pm 3$ \\
\hline SMC5_051315 & $2.4 \pm 0.4$ & $3.1 \pm 0.5$ & $2.8 \pm 0.5$ & $262 \pm 10$ \\
\hline SMC5_052688 & $4.2 \pm 0.4$ & $9.6 \pm 0.5$ & $12.8 \pm 1.5$ & $27 \pm 3$ \\
\hline SMC5_053267 & $3.5 \pm 0.4$ & $6.0 \pm 0.5$ & $5.0 \pm 1.0$ & $68 \pm 6$ \\
\hline SMC5_053756 & $3.7 \pm 0.4$ & $6.8 \pm 0.5$ & $6.0 \pm 1.0$ & $50 \pm 6$ \\
\hline SMC5_055592 & $3.5 \pm 0.4$ & $5.8 \pm 0.5$ & $6.5 \pm 1.0$ & $71 \pm 6$ \\
\hline SMC5_061950 & $3.1 \pm 0.4$ & $4.3 \pm 0.5$ & $6.2 \pm 1.0$ & $127 \pm 6$ \\
\hline SMC5_064327 & $4.6 \pm 0.4$ & $12.9 \pm 1.0$ & $24.9 \pm 2.0$ & $17 \pm 3$ \\
\hline SMC5_064576 & $3.2 \pm 0.4$ & $5.6 \pm 0.5$ & $3.1 \pm 0.5$ & $56 \pm 6$ \\
\hline SMC5_064745 & $5.2 \pm 0.4$ & $22.8 \pm 1.5$ & $15.2 \pm 1.5$ & $8 \pm 1$ \\
\hline SMC5_064832 & $3.5 \pm 0.4$ & $6.0 \pm 0.5$ & $8.4 \pm 1.0$ & $68 \pm 6$ \\
\hline SMC5_065055 & $5.0 \pm 0.4$ & $18.0 \pm 1.0$ & $18.3 \pm 1.5$ & $11 \pm 3$ \\
\hline SMC5_065746 & $3.5 \pm 0.4$ & $7.2 \pm 0.5$ & $3.3 \pm 0.5$ & $26 \pm 3$ \\
\hline SMC5_066754 & $3.7 \pm 0.4$ & $7.0 \pm 0.5$ & $6.7 \pm 1.0$ & $45 \pm 3$ \\
\hline SMC5_067333 & $3.5 \pm 0.4$ & $6.1 \pm 0.5$ & $5.0 \pm 1.0$ & $65 \pm 6$ \\
\hline SMC5_073581 & $3.9 \pm 0.4$ & $7.5 \pm 0.5$ & $9.9 \pm 1.0$ & $41 \pm 3$ \\
\hline SMC5_073594 & $3.7 \pm 0.4$ & $6.6 \pm 0.5$ & $7.4 \pm 1.0$ & $53 \pm 6$ \\
\hline SMC5_074402 & $4.4 \pm 0.4$ & $11.2 \pm 1.0$ & $16.9 \pm 1.5$ & $21 \pm 3$ \\
\hline SMC5_074471 & $4.3 \pm 0.4$ & $10.7 \pm 1.0$ & $14.8 \pm 1.5$ & $23 \pm 3$ \\
\hline SMC5_075061 & $3.0 \pm 0.4$ & $4.2 \pm 0.5$ & $4.2 \pm 0.5$ & $134 \pm 6$ \\
\hline SMC5_075360 & $3.8 \pm 0.4$ & $7.5 \pm 0.5$ & $7.7 \pm 1.0$ & $41 \pm 3$ \\
\hline SMC5_078338 & $4.0 \pm 0.4$ & $8.5 \pm 0.5$ & $9.5 \pm 1.0$ & $33 \pm 3$ \\
\hline SMC5_078440 & $4.8 \pm 0.4$ & $14.6 \pm 1.0$ & $24.9 \pm 2.0$ & $14 \pm 3$ \\
\hline SMC5_078928 & $3.7 \pm 0.4$ & $6.7 \pm 0.5$ & $6.5 \pm 1.0$ & $52 \pm 6$ \\
\hline SMC5_080910 & $3.7 \pm 0.4$ & $6.7 \pm 0.5$ & $5.9 \pm 1.0$ & $51 \pm 6$ \\
\hline SMC5_081260 & $2.4 \pm 0.4$ & $3.3 \pm 0.5$ & $2.4 \pm 1.0$ & $200 \pm 10$ \\
\hline SMC5_082042 & $4.7 \pm 0.4$ & $13.9 \pm 1.0$ & $25.1 \pm 2.0$ & $15 \pm 3$ \\
\hline SMC5_082202 & $3.5 \pm 0.4$ & $5.6 \pm 0.5$ & $8.2 \pm 1.0$ & $75 \pm 6$ \\
\hline
\end{tabular}


C. Martayan et al.: B and Be stars in the SMC. II., Online Material p 15

Table 4. continued.

\begin{tabular}{ccccc}
\hline \hline Star & $\log \left(L / L_{\odot}\right)$ & $M / M_{\odot}$ & $R / R_{\odot}$ & age Myears \\
\hline SMC5_082543 & $3.8 \pm 0.4$ & $8.0 \pm 0.5$ & $5.3 \pm 1.0$ & $34 \pm 3$ \\
SMC5_082819 & $5.1 \pm 0.4$ & $18.6 \pm 1.0$ & $28.4 \pm 2.5$ & $10 \pm 3$ \\
SMC5_082941 & $4.2 \pm 0.4$ & $9.8 \pm 1.0$ & $14.8 \pm 1.5$ & $26 \pm 3$ \\
SMC5_083491 & $4.6 \pm 0.4$ & $12.6 \pm 1.0$ & $21.7 \pm 2.0$ & $17 \pm 3$ \\
SMC5_085503 & $4.0 \pm 0.4$ & $7.9 \pm 0.5$ & $14.6 \pm 1.5$ & $38 \pm 3$ \\
SMC5_086200 & $3.6 \pm 0.4$ & $6.3 \pm 0.5$ & $5.7 \pm 1.0$ & $61 \pm 6$ \\
SMC5_086251 & $3.6 \pm 0.4$ & $6.5 \pm 0.5$ & $5.6 \pm 1.0$ & $55 \pm 6$ \\
SMC5_086581 & $2.7 \pm 0.4$ & $3.6 \pm 0.5$ & $3.3 \pm 1.0$ & $196 \pm 10$ \\
SMC5_086890 & $3.9 \pm 0.4$ & $8.7 \pm 0.5$ & $5.3 \pm 1.0$ & $29 \pm 3$ \\
SMC5_086983 & $4.0 \pm 0.4$ & $8.3 \pm 0.5$ & $7.0 \pm 1.0$ & $34 \pm 3$ \\
SMC5_087004 & $3.7 \pm 0.4$ & $7.7 \pm 0.5$ & $4.1 \pm 0.5$ & $32 \pm 3$ \\
SMC5_090914 & $3.9 \pm 0.4$ & $7.5 \pm 0.5$ & $10.9 \pm 1.5$ & $41 \pm 3$ \\
SMC5_190576 & $5.7 \pm 0.4$ & $38.1 \pm 2.5$ & $26.6 \pm 2.0$ & $5 \pm 1$ \\
\hline
\end{tabular}


Table 5. Fundamental parameters for Be stars in the SMC corrected from the effects of fast rotation assuming different rotation rates $\left(\Omega / \Omega_{\mathrm{c}}\right)$. The most suitable corrections are those corresponding to $\Omega / \Omega_{\mathrm{c}}=95 \%$. The units are $\mathrm{K}$ for $T_{\mathrm{eff}}^{\mathrm{o}}$, dex for $\log g_{\mathrm{o}}$, and $\mathrm{km} \mathrm{s}^{-1}$ for $V \sin i^{\text {true }}$.

\begin{tabular}{|c|c|c|c|c|c|c|c|c|c|}
\hline \multirow{2}{*}{$\begin{array}{l}\text { star } \\
\text { SMC }\end{array}$} & \multirow[b]{2}{*}{$T_{\mathrm{eff}}^{\mathrm{o}}$} & \multicolumn{2}{|c|}{$\bar{\Omega} \Omega / \Omega_{\mathrm{c}}=85 \%$} & \multicolumn{3}{|c|}{$\bar{\Omega} \Omega / \Omega_{\mathrm{c}}=90 \%$} & \multirow[b]{2}{*}{$T_{\mathrm{eff}}^{\mathrm{o}}$} & \multicolumn{2}{|c|}{$\bar{\Omega} \Omega / \Omega_{\mathrm{c}}=95 \%$} \\
\hline & & $\log g_{\mathrm{o}}$ & $V \sin i^{\text {true }}$ & $T_{\mathrm{eff}}^{\mathrm{o}}$ & $\log g_{\mathrm{o}}$ & $V \sin i^{\text {true }}$ & & $\log g_{\mathrm{o}}$ & $V \sin i^{\text {true }}$ \\
\hline MHF[S9]47315 & $33000 \pm 750$ & $3.7 \pm 0.1$ & $383 \pm 19$ & $34000 \pm 750$ & $3.7 \pm 0.1$ & $390 \pm 19$ & $32500 \pm 750$ & $3.8 \pm 0.1$ & $396 \pm 19$ \\
\hline MHF[S9]51066 & $26500 \pm 1100$ & $3.7 \pm 0.1$ & $428 \pm 21$ & $27500 \pm 1100$ & $3.8 \pm 0.1$ & $437 \pm 21$ & $26000 \pm 1100$ & $3.8 \pm 0.1$ & $450 \pm 21$ \\
\hline SMC5_000476 & $20000 \pm 2800$ & $4.3 \pm 0.2$ & $321 \pm 61$ & $20000 \pm 2800$ & $4.3 \pm 0.2$ & $328 \pm 61$ & $20000 \pm 2800$ & $4.3 \pm 0.2$ & $336 \pm 61$ \\
\hline SMC5_000643 & $19000 \pm 700$ & $3.7 \pm 0.2$ & $292 \pm 28$ & $19000 \pm 700$ & $3.7 \pm 0.2$ & $297 \pm 28$ & $19500 \pm 700$ & $3.8 \pm 0.2$ & $301 \pm 28$ \\
\hline SMC5_002232 & $21500 \pm 1200$ & $3.3 \pm 0.1$ & $252 \pm 17$ & $22000 \pm 1200$ & $3.4 \pm 0.1$ & $256 \pm 17$ & $22000 \pm 1200$ & $3.4 \pm 0.1$ & $261 \pm 17$ \\
\hline SMC5_002483 & $21500 \pm 3100$ & $4.1 \pm 0.2$ & $363 \pm 70$ & $21500 \pm 3100$ & $4.2 \pm 0.2$ & $368 \pm 70$ & $21500 \pm 3100$ & $4.2 \pm 0.2$ & $374 \pm 70$ \\
\hline SMC5_002751 & $18500 \pm 2200$ & $3.5 \pm 0.2$ & $328 \pm 54$ & $18500 \pm 2200$ & $3.5 \pm 0.2$ & $334 \pm 54$ & $19000 \pm 2200$ & $3.5 \pm 0.2$ & $342 \pm 54$ \\
\hline SMC5_002825 & $25500 \pm 2900$ & $4.6 \pm 0.2$ & $283 \pm 44$ & $25500 \pm 2900$ & $4.7 \pm 0.2$ & $288 \pm 44$ & $26000 \pm 2900$ & $4.7 \pm 0.2$ & $288 \pm 44$ \\
\hline SMC5_002957 & $19000 \pm 900$ & $3.3 \pm 0.1$ & $250 \pm 12$ & $19500 \pm 900$ & $3.3 \pm 0.1$ & $254 \pm 12$ & $19500 \pm 900$ & $3.7 \pm 0.1$ & $259 \pm 12$ \\
\hline SMC5_002984 & $15500 \pm 2900$ & $3.8 \pm 0.2$ & $326 \pm 47$ & $16000 \pm 2900$ & $3.8 \pm 0.2$ & $341 \pm 47$ & $16000 \pm 2900$ & $3.8 \pm 0.2$ & $354 \pm 47$ \\
\hline SMC5_003119 & $18500 \pm 1600$ & $3.3 \pm 0.1$ & $277 \pm 42$ & $18500 \pm 1600$ & $3.3 \pm 0.1$ & $281 \pm 42$ & $19000 \pm 1600$ & $3.5 \pm 0.1$ & $286 \pm 42$ \\
\hline SMC5_003296 & $20500 \pm 1600$ & $4.0 \pm 0.2$ & $192 \pm 18$ & $20500 \pm 1600$ & $4.1 \pm 0.2$ & $192 \pm 18$ & $21000 \pm 1600$ & $4.1 \pm 0.2$ & $196 \pm 18$ \\
\hline SMC5_003315 & $19000 \pm 2900$ & $3.6 \pm 0.2$ & \pm 48 & $19500 \pm 2900$ & $3.6 \pm 0.2$ & $7 \pm 48$ & $20000 \pm 2900$ & $3.6 \pm 0.2$ & $323 \pm 48$ \\
\hline SMC5_003389 & $20500 \pm 750$ & $3.3 \pm 0.2$ & $8 \pm 31$ & $21000 \pm 750$ & $3.4 \pm 0.2$ & $329 \pm 31$ & $21500 \pm 750$ & $3.4 \pm 0.2$ & $338 \pm 31$ \\
\hline SMC5_003537 & $21500 \pm 2500$ & $3.7 \pm 0.2$ & $4 \pm 32$ & $21500 \pm 2500$ & $3.7 \pm 0.2$ & $206 \pm 32$ & $22000 \pm 2500$ & $3.7 \pm 0.2$ & $211 \pm 32$ \\
\hline SMC5_003789 & $27500 \pm 4800$ & $4.5 \pm 0.2$ & $509 \pm 75$ & $27500 \pm 4800$ & $4.5 \pm 0.2$ & $517 \pm 75$ & $27500 \pm 4800$ & $4.6 \pm 0.2$ & $528 \pm 75$ \\
\hline SMC5_003919 & $25000 \pm 3800$ & $4.3 \pm 0.2$ & $321 \pm 62$ & $26000 \pm 3800$ & $4.2 \pm 0.2$ & $324 \pm 62$ & $25500 \pm 3800$ & $4.4 \pm 0.2$ & $326 \pm 62$ \\
\hline SMC5_004026 & $28500 \pm 4300$ & $4.2 \pm 0.2$ & $510 \pm 75$ & $28000 \pm 4300$ & $4.2 \pm 0.2$ & $515 \pm 75$ & $28000 \pm 4300$ & $4.2 \pm 0.2$ & $536 \pm 75$ \\
\hline SMC5_004201 & $16500 \pm 2800$ & $3.6 \pm 0.1$ & $500 \pm 72$ & $17500 \pm 2800$ & $3.6 \pm 0.1$ & $518 \pm 72$ & $18500 \pm 2800$ & $3.6 \pm 0.1$ & $550 \pm 72$ \\
\hline SMC5_004509 & $19500 \pm 1900$ & $3.8 \pm 0.2$ & $109 \pm 16$ & $20000 \pm 1900$ & $3.8 \pm 0.2$ & $110 \pm 16$ & $20000 \pm 1900$ & $3.8 \pm 0.2$ & $113 \pm 16$ \\
\hline SMC5_004685 & $18500 \pm 2600$ & $3.8 \pm 0.2$ & $259 \pm 49$ & $18500 \pm 2600$ & $3.9 \pm 0.2$ & $264 \pm 49$ & $19000 \pm 2600$ & $3.9 \pm 0.2$ & $271 \pm 49$ \\
\hline SMC5_004982 & $21000 \pm 3000$ & $4.0 \pm 0.2$ & $280 \pm 54$ & $21500 \pm 3000$ & $4.0 \pm 0.2$ & $281 \pm 54$ & $21500 \pm 3000$ & $4.1 \pm 0.2$ & $288 \pm 54$ \\
\hline SMC5_005045 & $20500 \pm 1800$ & $4.1 \pm 0.2$ & $236 \pm 27$ & $21000 \pm 1800$ & $4.1 \pm 0.2$ & $243 \pm 27$ & $21000 \pm 1800$ & $4.2 \pm 0.2$ & $241 \pm 27$ \\
\hline SMC5_008231 & $23000 \pm 2100$ & $3.5 \pm 0.2$ & \pm 52 & $23000 \pm 2100$ & $3.5 \pm 0.2$ & $335 \pm 52$ & 2100 & $3.5 \pm 0.2$ & $338 \pm 52$ \\
\hline SMC5_009378 & $18000 \pm 3300$ & $3.7 \pm 0.2$ & \pm 50 & $18500 \pm 3300$ & $3.8 \pm 0.2$ & $370 \pm 50$ & 3300 & $3.8 \pm 0.2$ & $382 \pm 50$ \\
\hline SMC5_011371 & $27000 \pm 4800$ & $4.1 \pm 0.2$ & \pm 70 & $27000 \pm 4800$ & $4.1 \pm 0.2$ & \pm 70 & $27500 \pm 4800$ & $4.5 \pm 0.2$ & $=70$ \\
\hline SMC5_01 & \pm 2000 & $3.8 \pm 0.2$ & 31 & 2000 & $3.8 \pm 0.2$ & 31 & 000 & $3.8 \pm 0.2$ & 31 \\
\hline SMC5_012717 & $20000 \pm 3600$ & $4.1 \pm 0.2$ & $374 \pm 54$ & $20000 \pm 3600$ & $4.0 \pm 0.2$ & $376 \pm 54$ & $20000 \pm 3600$ & $4.0 \pm 0.2$ & $382 \pm 54$ \\
\hline SMC5_012767 & $31500 \pm 5600$ & $3.9 \pm 0.2$ & $356 \pm 50$ & $30000 \pm 5600$ & $3.8 \pm 0.2$ & $361 \pm 50$ & $31500 \pm 5600$ & $3.9 \pm 0.2$ & $363 \pm 50$ \\
\hline SMC5_013233 & $15000 \pm 2800$ & $4.1 \pm 0.2$ & $280 \pm 40$ & $15000 \pm 2800$ & $4.1 \pm 0.2$ & $288 \pm 40$ & $14500 \pm 2800$ & $4.1 \pm 0.2$ & $293 \pm 40$ \\
\hline SMC5_013978 & $19000 \pm 2100$ & $3.5 \pm 0.2$ & $297 \pm 37$ & $18500 \pm 2100$ & $3.5 \pm 0.2$ & $296 \pm 37$ & $20000 \pm 2100$ & $3.6 \pm 0.2$ & $311 \pm 37$ \\
\hline SMC5_014052 & $19500 \pm 1600$ & $3.4 \pm 0.2$ & $104 \pm 10$ & $19500 \pm 1600$ & $3.4 \pm 0.2$ & $104 \pm 10$ & $20000 \pm 1600$ & $3.5 \pm 0.2$ & $107 \pm 10$ \\
\hline SMC5_014114 & $23000 \pm 700$ & $3.3 \pm 0.1$ & $123 \pm 10$ & $23000 \pm 700$ & $3.3 \pm 0.1$ & $124 \pm 10$ & $23000 \pm 700$ & $3.3 \pm 0.1$ & $125 \pm 10$ \\
\hline SMC5_014212 & $20000 \pm 500$ & $3.5 \pm 0.1$ & $222 \pm 11$ & $20500 \pm 500$ & $3.5 \pm 0.1$ & $225 \pm 11$ & $20500 \pm 500$ & $3.6 \pm 0.1$ & $229 \pm 11$ \\
\hline SMC5_014271 & $35500 \pm 1800$ & $3.7 \pm 0.1$ & $512 \pm 35$ & $38000 \pm 1800$ & $3.8 \pm 0.1$ & $525 \pm 35$ & $35000 \pm 1800$ & $4.0 \pm 0.1$ & $559 \pm 35$ \\
\hline SMC5_014637 & $19000 \pm 1900$ & $3.5 \pm 0.2$ & $172 \pm 16$ & $19500 \pm 1900$ & $3.5 \pm 0.2$ & $175 \pm 16$ & $19000 \pm 1900$ & $3.6 \pm 0.2$ & $176 \pm 16$ \\
\hline SMC5_014727 & $22500 \pm 2400$ & $3.8 \pm 0.2$ & $324 \pm 32$ & $22500 \pm 2400$ & $3.8 \pm 0.2$ & $331 \pm 32$ & $21500 \pm 2400$ & $4.0 \pm 0.2$ & $333 \pm 32$ \\
\hline SMC5_014864 & $17000 \pm 500$ & $3.5 \pm 0.1$ & $114 \pm 10$ & $17000 \pm 500$ & $3.5 \pm 0.1$ & $116 \pm 10$ & $17000 \pm 500$ & $3.6 \pm 0.1$ & $121 \pm 10$ \\
\hline SMC5_014878 & $25500 \pm 500$ & $3.7 \pm 0.1$ & $419 \pm 20$ & $27500 \pm 500$ & $3.7 \pm 0.1$ & $398 \pm 20$ & $25000 \pm 500$ & $3.7 \pm 0.1$ & $442 \pm 20$ \\
\hline SMC5_015429 & $30000 \pm 5000$ & $4.9 \pm 0.2$ & $480 \pm 70$ & $29000 \pm 5000$ & $4.9 \pm 0.2$ & $489 \pm 70$ & $28000 \pm 5000$ & $4.9 \pm 0.2$ & $497 \pm 70$ \\
\hline SMC5_015509 & $26500 \pm 4300$ & $4.3 \pm 0.2$ & $490 \pm 71$ & $25500 \pm 4300$ & $4.3 \pm 0.2$ & $495 \pm 71$ & $24500 \pm 4300$ & $4.3 \pm 0.2$ & $509 \pm 71$ \\
\hline SMC5_015867 & $21000 \pm 1600$ & $4.0 \pm 0.2$ & $217 \pm 21$ & $21000 \pm 1600$ & $4.1 \pm 0.2$ & $218 \pm 21$ & $21000 \pm 1600$ & $4.1 \pm 0.2$ & $218 \pm 21$ \\
\hline SMC5_016177 & $25500 \pm 2500$ & $4.3 \pm 0.2$ & $292 \pm 29$ & $26500 \pm 2500$ & $4.2 \pm 0.2$ & $296 \pm 29$ & $25500 \pm 2500$ & $4.2 \pm 0.2$ & $297 \pm 29$ \\
\hline SMC5_016461 & $18500 \pm 700$ & $2.9 \pm 0.1$ & $325 \pm 31$ & $18500 \pm 700$ & $2.8 \pm 0.1$ & $327 \pm 31$ & $18500 \pm 700$ & $2.9 \pm 0.1$ & $335 \pm 31$ \\
\hline SMC5_016477 & $11500 \pm 2200$ & $3.5 \pm 0.2$ & $318 \pm 45$ & $11500 \pm 2200$ & $3.5 \pm 0.2$ & $317 \pm 45$ & $11000 \pm 2200$ & $3.6 \pm 0.2$ & $326 \pm 45$ \\
\hline SMC5_016486 & $30500 \pm 1300$ & $4.1 \pm 0.1$ & $509 \pm 25$ & $32500 \pm 1300$ & $4.1 \pm 0.1$ & $522 \pm 25$ & $30000 \pm 1300$ & $4.0 \pm 0.1$ & $555 \pm 25$ \\
\hline SMC5_016523 & $23000 \pm 2000$ & $3.5 \pm 0.2$ & $445 \pm 43$ & $23000 \pm 2000$ & $3.6 \pm 0.2$ & $451 \pm 43$ & $23000 \pm 2000$ & $3.7 \pm 0.2$ & $454 \pm 43$ \\
\hline SMC5_016544 & $23000 \pm 2100$ & $3.9 \pm 0.2$ & $352 \pm 34$ & $23000 \pm 2100$ & $3.9 \pm 0.2$ & $355 \pm 34$ & $23500 \pm 2100$ & $4.0 \pm 0.2$ & $357 \pm 34$ \\
\hline
\end{tabular}


Table 5. continued.

\begin{tabular}{|c|c|c|c|c|c|c|c|c|c|}
\hline \multirow{2}{*}{$\begin{array}{l}\text { star } \\
\text { SMC }\end{array}$} & \multirow[b]{2}{*}{$T_{\mathrm{eff}}^{\mathrm{o}}$} & \multicolumn{2}{|c|}{$\bar{\Omega} / \Omega_{\mathrm{c}}=85 \%$} & \multicolumn{3}{|c|}{$\Omega / \Omega_{\mathrm{c}}=90 \%$} & \multirow[b]{2}{*}{$T_{\mathrm{eff}}^{\mathrm{o}}$} & \multicolumn{2}{|c|}{$\overline{\Omega / \Omega_{\mathrm{c}}=95 \%}$} \\
\hline & & $\log g_{\mathrm{o}}$ & $V \sin i^{\text {true }}$ & $T_{\mathrm{eff}}^{\mathrm{o}}$ & $\log g_{\mathrm{o}}$ & $V \sin i^{\text {true }}$ & & $\log g_{\mathrm{o}}$ & $V \sin i^{\text {true }}$ \\
\hline SMC5_016824 & $10500 \pm 800$ & $3.7 \pm 0.2$ & $200 \pm 10$ & $10500 \pm 800$ & $3.7 \pm 0.2$ & $203 \pm 10$ & $10500 \pm 800$ & $3.7 \pm 0.2$ & $205 \pm 10$ \\
\hline SMC5_017596 & $20000 \pm 1900$ & $4.0 \pm 0.2$ & $213 \pm 20$ & $19500 \pm 1900$ & $4.0 \pm 0.2$ & $210 \pm 20$ & $20500 \pm 1900$ & $4.0 \pm 0.2$ & $218 \pm 20$ \\
\hline SMC5_018501 & $26000 \pm 1200$ & $4.0 \pm 0.2$ & $197 \pm 19$ & $24000 \pm 1200$ & $4.0 \pm 0.2$ & $373 \pm 19$ & $25500 \pm 1200$ & $4.0 \pm 0.2$ & $373 \pm 19$ \\
\hline SMC5_010211 & $21700 \pm 1100$ & $3.8 \pm 0.2$ & $322 \pm 17$ & $23000 \pm 1100$ & $3.8 \pm 0.2$ & $323 \pm 17$ & $21700 \pm 1100$ & $3.8 \pm 0.2$ & $175 \pm 17$ \\
\hline SMC5_021152 & $19500 \pm 900$ & $3.3 \pm 0.2$ & $211 \pm 10$ & $19500 \pm 900$ & $3.3 \pm 0.2$ & $214 \pm 10$ & $19500 \pm 900$ & $3.3 \pm 0.2$ & $218 \pm 10$ \\
\hline SMC5_021886 & $22200 \pm 1100$ & $3.8 \pm 0.2$ & $211 \pm 21$ & $22200 \pm 1100$ & $4.1 \pm 0.2$ & $212 \pm 21$ & $26000 \pm 1100$ & $4.2 \pm 0.2$ & $432 \pm 21$ \\
\hline SMC5_022295 & $22500 \pm 1000$ & $3.3 \pm 0.2$ & $369 \pm 22$ & $21500 \pm 1000$ & $3.3 \pm 0.2$ & $363 \pm 22$ & $22500 \pm 1000$ & $3.3 \pm 0.2$ & $392 \pm 22$ \\
\hline SMC5_022628 & $27000 \pm 1100$ & $3.9 \pm 0.2$ & $230 \pm 23$ & $29000 \pm 1100$ & $3.9 \pm 0.2$ & $481 \pm 23$ & $27000 \pm 1100$ & $4.2 \pm 0.2$ & $515 \pm 23$ \\
\hline SMC5_022842 & $30000 \pm 2100$ & $4.2 \pm 0.2$ & $510 \pm 50$ & $30500 \pm 2100$ & $4.2 \pm 0.2$ & $515 \pm 50$ & $26500 \pm 2100$ & $4.2 \pm 0.2$ & $551 \pm 50$ \\
\hline SMC5_023931 & $22000 \pm 1600$ & $3.9 \pm 0.2$ & $357 \pm 17$ & $22000 \pm 1600$ & $3.9 \pm 0.2$ & $360 \pm 17$ & $22000 \pm 1600$ & $4.0 \pm 0.2$ & $368 \pm 17$ \\
\hline SMC5_025052 & $17000 \pm 2800$ & $3.8 \pm 0.2$ & $369 \pm 50$ & $17500 \pm 2800$ & $3.8 \pm 0.2$ & $371 \pm 50$ & $17500 \pm 2800$ & $3.9 \pm 0.2$ & $375 \pm 50$ \\
\hline SMC5_025589 & $20500 \pm 2300$ & $4.2 \pm 0.2$ & $370 \pm 50$ & $20000 \pm 2300$ & $4.2 \pm 0.2$ & $376 \pm 50$ & $18000 \pm 2300$ & $4.5 \pm 0.2$ & $398 \pm 50$ \\
\hline SMC5_025718 & $23000 \pm 2000$ & $3.4 \pm 0.2$ & $275 \pm 17$ & $21500 \pm 2000$ & $3.4 \pm 0.2$ & $274 \pm 17$ & $21500 \pm 2000$ & $3.6 \pm 0.2$ & $280 \pm 17$ \\
\hline SMC5_025816 & $19000 \pm 1800$ & $3.5 \pm 0.2$ & $233 \pm 11$ & $19000 \pm 1800$ & $3.5 \pm 0.2$ & $235 \pm 11$ & $19500 \pm 1800$ & $3.5 \pm 0.2$ & $244 \pm 11$ \\
\hline SMC5_025829 & $21000 \pm 1100$ & $3.5 \pm 0.2$ & $277 \pm 17$ & $20000 \pm 1100$ & $3.8 \pm 0.2$ & $278 \pm 17$ & $20500 \pm 1100$ & $3.8 \pm 0.2$ & $286 \pm 17$ \\
\hline SMC5_026182 & $18500 \pm 3000$ & $4.2 \pm 0.2$ & $384 \pm 54$ & $17500 \pm 3000$ & $4.3 \pm 0.2$ & $382 \pm 54$ & $17000 \pm 3000$ & $4.4 \pm 0.2$ & $402 \pm 54$ \\
\hline SMC5_026689 & $24000 \pm 1900$ & $4.5 \pm 0.2$ & $407 \pm 59$ & $22000 \pm 1900$ & $4.6 \pm 0.2$ & $416 \pm 59$ & $22500 \pm 1900$ & $4.6 \pm 0.2$ & $425 \pm 59$ \\
\hline SMC5_028368 & $23500 \pm 1800$ & $3.7 \pm 0.2$ & $529 \pm 70$ & $22500 \pm 1800$ & $3.8 \pm 0.2$ & $532 \pm 70$ & $22500 \pm 1800$ & $4.0 \pm 0.2$ & $541 \pm 70$ \\
\hline SMC5_036967 & $22000 \pm 1400$ & $3.5 \pm 0.2$ & $326 \pm 20$ & $20000 \pm 1400$ & $3.5 \pm 0.2$ & $329 \pm 20$ & $20000 \pm 1400$ & $3.5 \pm 0.2$ & $334 \pm 20$ \\
\hline SMC5_037013 & $20000 \pm 500$ & $3.3 \pm 0.1$ & $144 \pm 10$ & $20000 \pm 500$ & $3.3 \pm 0.1$ & $145 \pm 10$ & $20000 \pm 500$ & $3.3 \pm 0.1$ & $148 \pm 10$ \\
\hline SMC5_037137 & $21500 \pm 600$ & $3.7 \pm 0.1$ & $292 \pm 10$ & $22000 \pm 600$ & $3.8 \pm 0.1$ & $296 \pm 10$ & $22000 \pm 600$ & $3.8 \pm 0.1$ & $301 \pm 10$ \\
\hline SMC5_037158 & $27500 \pm 2300$ & $3.9 \pm 0.2$ & $435 \pm 60$ & $26000 \pm 2300$ & $3.9 \pm 0.2$ & $441 \pm 60$ & $26000 \pm 2300$ & $4.1 \pm 0.2$ & $448 \pm 60$ \\
\hline SMC5_037162 & $24000 \pm 500$ & $3.7 \pm 0.2$ & $378 \pm 55$ & $22500 \pm 500$ & $3.8 \pm 0.2$ & $381 \pm 55$ & $23000 \pm 500$ & $3.9 \pm 0.2$ & $386 \pm 55$ \\
\hline SMC5_038007 & $18000 \pm 900$ & $4.2 \pm 0.2$ & $279 \pm 13$ & $17500 \pm 900$ & $4.3 \pm 0.2$ & $283 \pm 13$ & $18000 \pm 900$ & $4.2 \pm 0.2$ & $293 \pm 13$ \\
\hline SMC5_038312 & $14000 \pm 1300$ & $3.6 \pm 0.2$ & $255 \pm 10$ & $13500 \pm 1300$ & $3.8 \pm 0.2$ & $274 \pm 10$ & $13500 \pm 1300$ & $3.7 \pm 0.2$ & $285 \pm 10$ \\
\hline SMC5_038363 & $25000 \pm 700$ & $4.0 \pm 0.1$ & $364 \pm 10$ & $24000 \pm 700$ & $4.0 \pm 0.1$ & $369 \pm 10$ & $25000 \pm 700$ & $4.0 \pm 0.1$ & $372 \pm 10$ \\
\hline SMC5_041410 & $25000 \pm 900$ & $3.5 \pm 0.2$ & $513 \pm 10$ & $25500 \pm 900$ & $3.6 \pm 0.2$ & $522 \pm 10$ & $25500 \pm 900$ & $3.5 \pm 0.2$ & $533 \pm 10$ \\
\hline SMC5_043413 & $21000 \pm 1900$ & $3.9 \pm 0.2$ & $5 \pm 41$ & $21000 \pm 1900$ & $3.9 \pm 0.2$ & $287 \pm 41$ & $21000 \pm 1900$ & $4.0 \pm 0.2$ & $294 \pm 41$ \\
\hline SMC5_044117 & $22000 \pm 500$ & $4.1 \pm 0.2$ & $344 \pm 50$ & $21500 \pm 500$ & $4.1 \pm 0.2$ & $346 \pm 50$ & $22000 \pm 500$ & $4.1 \pm 0.2$ & $351 \pm 50$ \\
\hline SMC5_044693 & $20500 \pm 600$ & $3.6 \pm 0.1$ & $269 \pm 10$ & $21500 \pm 600$ & $3.6 \pm 0.1$ & $274 \pm 10$ & $21500 \pm 600$ & $3.6 \pm 0.1$ & $=10$ \\
\hline SMC5_044898 & \pm 800 & $3.6 \pm 0.2$ & 19 & 00 & $3.6 \pm 0.2$ & 19 & 00 & $3.6 \pm 0.2$ & 19 \\
\hline SMC5_045353 & $21500 \pm 500$ & $3.5 \pm 0.1$ & $332 \pm 10$ & $21500 \pm 500$ & $3.5 \pm 0.1$ & $335 \pm 10$ & $21500 \pm 500$ & $3.6 \pm 0.1$ & $343 \pm 10$ \\
\hline SMC5_045747 & $20000 \pm 700$ & $3.7 \pm 0.2$ & $290 \pm 18$ & $20000 \pm 700$ & $3.7 \pm 0.2$ & $293 \pm 18$ & $20500 \pm 700$ & $3.8 \pm 0.2$ & $300 \pm 18$ \\
\hline SMC5_046388 & $22500 \pm 2000$ & $3.9 \pm 0.2$ & $336 \pm 50$ & $22000 \pm 2000$ & $3.9 \pm 0.2$ & $343 \pm 50$ & $22500 \pm 2000$ & $4.0 \pm 0.2$ & $347 \pm 50$ \\
\hline SMC5_046462 & $23000 \pm 1000$ & $4.0 \pm 0.2$ & $359 \pm 22$ & $22500 \pm 1000$ & $4.0 \pm 0.2$ & $359 \pm 22$ & $23000 \pm 1000$ & $4.0 \pm 0.2$ & $363 \pm 22$ \\
\hline SMC5_047763 & $13000 \pm 1000$ & $2.6 \pm 0.2$ & $97 \pm 12$ & $13000 \pm 1000$ & $2.6 \pm 0.2$ & $100 \pm 12$ & $13000 \pm 1000$ & $2.6 \pm 0.2$ & $101 \pm 12$ \\
\hline SMC5_048045 & $22500 \pm 700$ & $3.5 \pm 0.2$ & $352 \pm 21$ & $21000 \pm 700$ & $3.6 \pm 0.2$ & $341 \pm 21$ & $20500 \pm 700$ & $3.6 \pm 0.2$ & $354 \pm 21$ \\
\hline SMC5_048047 & $20500 \pm 300$ & $3.6 \pm 0.1$ & $233 \pm 11$ & $20500 \pm 300$ & $3.7 \pm 0.1$ & $237 \pm 11$ & $21000 \pm 300$ & $3.7 \pm 0.1$ & $238 \pm 11$ \\
\hline SMC5_048289 & $20500 \pm 1100$ & $4.0 \pm 0.2$ & $263 \pm 13$ & $20500 \pm 1100$ & $4.1 \pm 0.2$ & $265 \pm 13$ & $21000 \pm 1100$ & $4.1 \pm 0.2$ & $270 \pm 13$ \\
\hline SMC5_049651 & $15000 \pm 1100$ & $4.3 \pm 0.2$ & $300 \pm 19$ & $15000 \pm 1100$ & $4.3 \pm 0.2$ & $311 \pm 19$ & $15000 \pm 1100$ & $4.3 \pm 0.2$ & $317 \pm 19$ \\
\hline SMC5_049746 & $23000 \pm 1000$ & $4.0 \pm 0.2$ & $453 \pm 60$ & $23500 \pm 1000$ & $4.1 \pm 0.2$ & $452 \pm 60$ & $22500 \pm 1000$ & $4.0 \pm 0.2$ & $466 \pm 60$ \\
\hline SMC5_049780 & $26000 \pm 1200$ & $3.8 \pm 0.2$ & $459 \pm 22$ & $26000 \pm 1200$ & $4.0 \pm 0.2$ & $465 \pm 22$ & $26500 \pm 1200$ & $4.0 \pm 0.2$ & $473 \pm 22$ \\
\hline SMC5_049996 & $24000 \pm 1400$ & $4.0 \pm 0.2$ & $477 \pm 30$ & $24000 \pm 1400$ & $4.1 \pm 0.2$ & $477 \pm 30$ & $23000 \pm 1400$ & $4.0 \pm 0.2$ & $490 \pm 30$ \\
\hline SMC5_051315 & $14000 \pm 1300$ & $4.1 \pm 0.2$ & $150 \pm 21$ & $14000 \pm 1300$ & $4.1 \pm 0.2$ & $150 \pm 21$ & $14000 \pm 1300$ & $4.1 \pm 0.2$ & $156 \pm 21$ \\
\hline SMC5_052688 & $19500 \pm 700$ & $3.5 \pm 0.2$ & $290 \pm 14$ & $20000 \pm 700$ & $3.5 \pm 0.2$ & $290 \pm 14$ & $20500 \pm 700$ & $3.7 \pm 0.2$ & $300 \pm 14$ \\
\hline SMC5_053267 & $23000 \pm 1000$ & $4.1 \pm 0.2$ & $466 \pm 65$ & $22500 \pm 1000$ & $4.1 \pm 0.2$ & $480 \pm 65$ & $25000 \pm 1000$ & $4.2 \pm 0.2$ & $493 \pm 65$ \\
\hline SMC5_053756 & $20000 \pm 800$ & $3.9 \pm 0.2$ & $155 \pm 10$ & $20500 \pm 800$ & $3.9 \pm 0.2$ & $157 \pm 10$ & $20500 \pm 800$ & $3.9 \pm 0.2$ & $160 \pm 10$ \\
\hline SMC5_055592 & $17000 \pm 1200$ & $3.7 \pm 0.2$ & $127 \pm 10$ & $17500 \pm 1200$ & $3.7 \pm 0.2$ & $129 \pm 10$ & $17500 \pm 1200$ & $3.7 \pm 0.2$ & $135 \pm 10$ \\
\hline SMC5_061950 & $15500 \pm 1300$ & $3.7 \pm 0.2$ & $297 \pm 45$ & $15500 \pm 1300$ & $3.7 \pm 0.2$ & $318 \pm 45$ & $15000 \pm 1300$ & $3.8 \pm 0.2$ & $332 \pm 45$ \\
\hline SMC5_064327 & $18500 \pm 500$ & $3.2 \pm 0.1$ & $297 \pm 14$ & $19000 \pm 500$ & $3.2 \pm 0.1$ & $299 \pm 14$ & $20000 \pm 500$ & $3.4 \pm 0.1$ & $312 \pm 14$ \\
\hline SMC5_064576 & $22000 \pm 1000$ & $4.5 \pm 0.2$ & $315 \pm 46$ & $22000 \pm 1000$ & $4.5 \pm 0.2$ & $321 \pm 46$ & $22000 \pm 1000$ & $4.5 \pm 0.2$ & $325 \pm 46$ \\
\hline SMC5_064745 & $34500 \pm 1000$ & $3.8 \pm 0.2$ & $460 \pm 36$ & $36000 \pm 1000$ & $3.8 \pm 0.2$ & $471 \pm 36$ & $34500 \pm 1000$ & $3.9 \pm 0.2$ & $495 \pm 36$ \\
\hline SMC5_064832 & $17500 \pm 800$ & $3.8 \pm 0.2$ & $316 \pm 15$ & $17500 \pm 800$ & $3.8 \pm 0.2$ & $316 \pm 15$ & $17500 \pm 800$ & $3.7 \pm 0.2$ & $336 \pm 15$ \\
\hline SMC5_065055 & $26500 \pm 900$ & $3.5 \pm 0.2$ & $431 \pm 21$ & $26500 \pm 900$ & $3.5 \pm 0.2$ & $435 \pm 21$ & $26500 \pm 900$ & $3.5 \pm 0.2$ & $443 \pm 21$ \\
\hline SMC5_065746 & $26000 \pm 1400$ & $4.5 \pm 0.2$ & $370 \pm 19$ & $27000 \pm 1400$ & $4.4 \pm 0.2$ & $375 \pm 19$ & $26000 \pm 1400$ & $4.5 \pm 0.2$ & $378 \pm 19$ \\
\hline SMC5_066754 & $19500 \pm 1400$ & $3.8 \pm 0.2$ & $174 \pm 10$ & $20000 \pm 1400$ & $3.8 \pm 0.2$ & $176 \pm 10$ & $20000 \pm 1400$ & $3.8 \pm 0.2$ & $178 \pm 10$ \\
\hline
\end{tabular}


Table 5. continued.

\begin{tabular}{|c|c|c|c|c|c|c|c|c|c|}
\hline \multirow{2}{*}{$\begin{array}{l}\text { star } \\
\text { SMC }\end{array}$} & \multicolumn{3}{|c|}{$\bar{\Omega} / \Omega_{\mathrm{c}}=85 \%$} & \multicolumn{3}{|c|}{$\bar{\Omega} / \Omega_{\mathrm{c}}=90 \%$} & \multicolumn{3}{|c|}{$\bar{\Omega} / \Omega_{\mathrm{c}}=95 \%$} \\
\hline & $T_{\mathrm{eff}}^{\mathrm{o}}$ & $\log g_{\mathrm{o}}$ & $V \sin i^{\text {true }}$ & $T_{\mathrm{eff}}^{\mathrm{o}}$ & $\log g_{\mathrm{o}}$ & $V \sin i^{\text {true }}$ & $T_{\mathrm{eff}}^{\mathrm{o}}$ & $\log g_{\mathrm{o}}$ & $V \sin i^{\text {true }}$ \\
\hline SMC5_067333 & $20500 \pm 1000$ & $4.1 \pm 0.2$ & $281 \pm 23$ & $20500 \pm 1000$ & $4.1 \pm 0.2$ & $285 \pm 23$ & $21000 \pm 1000$ & $4.1 \pm 0.2$ & $289 \pm 23$ \\
\hline SMC5_073581 & $19000 \pm 1200$ & $3.6 \pm 0.2$ & $240 \pm 15$ & $19000 \pm 1200$ & $3.7 \pm 0.2$ & $245 \pm 15$ & $19000 \pm 1200$ & $3.6 \pm 0.2$ & $251 \pm 15$ \\
\hline SMC5_073594 & $18500 \pm 1300$ & $3.7 \pm 0.2$ & $203 \pm 13$ & $19000 \pm 1300$ & $3.7 \pm 0.2$ & $207 \pm 13$ & $19000 \pm 1300$ & $3.8 \pm 0.2$ & $213 \pm 13$ \\
\hline SMC5_074402 & $20000 \pm 1100$ & $3.7 \pm 0.2$ & $403 \pm 20$ & $21000 \pm 1100$ & $3.7 \pm 0.2$ & $417 \pm 20$ & $22000 \pm 1100$ & $3.8 \pm 0.2$ & $437 \pm 20$ \\
\hline SMC5_074471 & $19500 \pm 700$ & $3.4 \pm 0.2$ & $196 \pm 10$ & $19500 \pm 700$ & $3.4 \pm 0.2$ & $197 \pm 10$ & $20000 \pm 700$ & $3.5 \pm 0.2$ & $201 \pm 10$ \\
\hline SMC5_075061 & $16000 \pm 1000$ & $3.9 \pm 0.2$ & $133 \pm 18$ & $16000 \pm 1000$ & $3.9 \pm 0.2$ & $133 \pm 18$ & $16000 \pm 1000$ & $3.9 \pm 0.2$ & $141 \pm 18$ \\
\hline SMC5_075360 & $20000 \pm 900$ & $3.8 \pm 0.2$ & $241 \pm 10$ & $20000 \pm 900$ & $3.8 \pm 0.2$ & $245 \pm 10$ & $20500 \pm 900$ & $3.9 \pm 0.2$ & $251 \pm 10$ \\
\hline SMC5_078338 & $19500 \pm 1400$ & $3.5 \pm 0.2$ & $79 \pm 10$ & $19500 \pm 1400$ & $3.5 \pm 0.2$ & $80 \pm 10$ & $19500 \pm 1400$ & $3.6 \pm 0.2$ & $83 \pm 10$ \\
\hline SMC5_078440 & $22500 \pm 900$ & $3.5 \pm 0.2$ & $358 \pm 17$ & $21000 \pm 900$ & $3.5 \pm 0.2$ & $369 \pm 17$ & $21000 \pm 900$ & $3.6 \pm 0.2$ & $378 \pm 17$ \\
\hline SMC5_078928 & $19500 \pm 1000$ & $3.8 \pm 0.2$ & $148 \pm 21$ & $19500 \pm 1000$ & $3.8 \pm 0.2$ & $150 \pm 21$ & $20000 \pm 1000$ & $3.8 \pm 0.2$ & $153 \pm 21$ \\
\hline SMC5_080910 & $21000 \pm 1000$ & $4.0 \pm 0.2$ & $324 \pm 15$ & $21000 \pm 1000$ & $4.1 \pm 0.2$ & $329 \pm 15$ & $21500 \pm 1000$ & $4.1 \pm 0.2$ & $334 \pm 15$ \\
\hline SMC5_081260 & $16000 \pm 1100$ & $4.4 \pm 0.2$ & $238 \pm 15$ & $16000 \pm 1100$ & $4.4 \pm 0.2$ & $249 \pm 15$ & $16000 \pm 1100$ & $4.4 \pm 0.2$ & $252 \pm 15$ \\
\hline SMC5_082042 & $19500 \pm 1300$ & $3.7 \pm 0.2$ & $420 \pm 22$ & $21000 \pm 1300$ & $3.7 \pm 0.2$ & $426 \pm 22$ & $21000 \pm 1300$ & $3.7 \pm 0.2$ & $433 \pm 22$ \\
\hline SMC5_082202 & $16000 \pm 300$ & $3.6 \pm 0.2$ & $277 \pm 13$ & $16000 \pm 300$ & $3.6 \pm 0.2$ & $287 \pm 13$ & $16500 \pm 300$ & $3.7 \pm 0.2$ & $298 \pm 13$ \\
\hline SMC5_082543 & $24500 \pm 1100$ & $4.2 \pm 0.2$ & $368 \pm 18$ & $27000 \pm 1100$ & $4.1 \pm 0.2$ & $378 \pm 18$ & $24500 \pm 1100$ & $4.2 \pm 0.2$ & $377 \pm 18$ \\
\hline SMC5_082819 & $21000 \pm 500$ & $3.1 \pm 0.1$ & $326 \pm 10$ & $21500 \pm 500$ & $3.1 \pm 0.1$ & $330 \pm 10$ & $23000 \pm 500$ & $3.1 \pm 0.1$ & $340 \pm 10$ \\
\hline SMC5_082941 & $18500 \pm 900$ & $3.5 \pm 0.2$ & $333 \pm 16$ & $19000 \pm 900$ & $3.5 \pm 0.2$ & $339 \pm 16$ & $21000 \pm 900$ & $3.7 \pm 0.2$ & $359 \pm 16$ \\
\hline SMC5_083491 & $20500 \pm 900$ & $3.5 \pm 0.2$ & 28 & 2000 & $3.5 \pm 0.2$ & \pm 28 & \pm 900 & $3.6 \pm 0.2$ & \pm 28 \\
\hline SMC5_08 & $15500 \pm$ & $3.3 \pm 0.1$ & 286 & 1600( & $3.2 \pm 0.1$ & 298 & 165 & $3.4 \pm 0.1$ & $297 \pm 14$ \\
\hline SMC5_086200 & $20500 \pm 1000$ & $4.1 \pm 0.2$ & $382 \pm 55$ & 21000 & $4.1 \pm 0.2$ & $387 \pm 55$ & 20000 & $4.1 \pm 0.2$ & $410 \pm 55$ \\
\hline SMC5_086251 & $20500 \pm 1400$ & $4.0 \pm 0.2$ & $304 \pm 19$ & $21000 \pm 1400$ & $4.1 \pm 0.2$ & $310 \pm 19$ & $21000 \pm 1400$ & $4.1 \pm 0.2$ & $314 \pm 19$ \\
\hline SMC5_086581 & $16000 \pm 900$ & $4.3 \pm 0.2$ & $319 \pm 15$ & $16500 \pm 900$ & $4.2 \pm 0.2$ & $334 \pm 15$ & $16000 \pm 900$ & $4.2 \pm 0.2$ & $336 \pm 15$ \\
\hline SMC5_086890 & $26000 \pm 900$ & $4.2 \pm 0.2$ & $392 \pm 19$ & $25500 \pm 900$ & $4.3 \pm 0.2$ & $395 \pm 19$ & $26000 \pm 900$ & $4.2 \pm 0.2$ & $400 \pm 19$ \\
\hline SMC5_086983 & $23500 \pm 1000$ & $4.0 \pm 0.2$ & $400 \pm 25$ & $24000 \pm 1000$ & $4.0 \pm 0.2$ & $405 \pm 25$ & $24000 \pm 1000$ & $4.0 \pm 0.2$ & $406 \pm 25$ \\
\hline SMC5_087004 & $25500 \pm 1200$ & $4.4 \pm 0.2$ & $393 \pm 19$ & $25500 \pm 1200$ & $4.5 \pm 0.2$ & $396 \pm 19$ & $26000 \pm 1200$ & $4.4 \pm 0.2$ & $401 \pm 19$ \\
\hline SMC5_090914 & $18000 \pm 1200$ & $3.6 \pm 0.2$ & $327 \pm 20$ & $18500 \pm 1200$ & $3.6 \pm 0.2$ & $329 \pm 20$ & $18500 \pm 1200$ & $3.5 \pm 0.2$ & $337 \pm 20$ \\
\hline SMC5_190576 & $32500 \pm 900$ & $3.5 \pm 0.1$ & $402 \pm 14$ & $32000 \pm 900$ & $3.4 \pm 0.1$ & $405 \pm 14$ & $32500 \pm 900$ & $3.4 \pm 0.1$ & $411 \pm 14$ \\
\hline
\end{tabular}


C. Martayan et al.: B and Be stars in the SMC. II., Online Material p 19

Table 6. Parameters: $\log \left(L / L_{\odot}\right), M / M_{\odot}, R / R_{\odot}$, and age of Be stars in the SMC, obtained by interpolation in the evolutionary tracks published in Schaller et al. (1992) with $Z=0.001$ with fundamental parameters corrected for fast rotation effects with $\Omega / \Omega_{\mathrm{c}}=95 \%$.

\begin{tabular}{|c|c|c|c|c|}
\hline$\overline{\text { Star }}$ & $\overline{l o g}\left(L / L_{\odot}\right)$ & $\overline{\bar{M} / M_{\odot}}$ & $\overline{R / R}$ & age Myears \\
\hline MHF[S9]47315 & $5.0 \pm 0.4$ & $21.3 \pm 1.5$ & $10.2 \pm 1.5$ & $8 \pm 1$ \\
\hline MHF[S9]51066 & $4.4 \pm 0.4$ & $12.0 \pm 1.0$ & $7.6 \pm 1.5$ & $18 \pm 3$ \\
\hline SMC5_000476 & $3.0 \pm 0.4$ & $5.0 \pm 0.5$ & $2.5 \pm 0.5$ & $51 \pm 6$ \\
\hline SMC5_000643 & $3.6 \pm 0.4$ & $6.6 \pm 0.5$ & $5.7 \pm 1.0$ & $55 \pm 6$ \\
\hline SMC5_002232 & $4.5 \pm 0.4$ & $11.9 \pm 1.0$ & $12.8 \pm 1.5$ & $18 \pm 3$ \\
\hline SMC5_002483 & $3.3 \pm 0.4$ & $6.2 \pm 0.5$ & $3.4 \pm 1.0$ & $47 \pm 3$ \\
\hline SMC5_002751 & $3.9 \pm 0.4$ & $7.5 \pm 0.5$ & $7.9 \pm 1.5$ & $41 \pm 3$ \\
\hline SMC5_002825 & - & - & - & - \\
\hline SMC5_002957 & $3.7 \pm 0.4$ & $6.7 \pm 1.0$ & $6.2 \pm 1.5$ & $51 \pm 6$ \\
\hline SMC5_002984 & $3.1 \pm 0.4$ & $4.6 \pm 0.5$ & $4.9 \pm 1.0$ & $111 \pm 6$ \\
\hline SMC5_003119 & $3.9 \pm 0.4$ & $7.8 \pm 0.5$ & $8.4 \pm 1.5$ & $39 \pm 3$ \\
\hline SMC5_003296 & $3.3 \pm 0.4$ & $6.1 \pm 0.5$ & $3.6 \pm 0.5$ & $53 \pm 6$ \\
\hline SMC5_003315 & $3.8 \pm 0.4$ & $7.5 \pm 0.5$ & $7.2 \pm 1.5$ & $41 \pm 3$ \\
\hline SMC5_003389 & $4.4 \pm 0.4$ & $11.1 \pm 1.0$ & $12.0 \pm 1.5$ & $21 \pm 3$ \\
\hline SMC5_003537 & $3.9 \pm 0.4$ & $8.2 \pm 0.5$ & $6.5 \pm 1.0$ & $35 \pm 3$ \\
\hline SMC5_003789 & $3.5 \pm 0.4$ & $7.3 \pm 0.5$ & $2.4 \pm 0.5$ & $27 \pm 3$ \\
\hline SMC5_003919 & $3.5 \pm 0.4$ & $7.8 \pm 0.5$ & $2.9 \pm 0.5$ & $13 \pm 3$ \\
\hline SMC5_004026 & $4.0 \pm 0.4$ & $10.6 \pm 0.5$ & $4.3 \pm 0.5$ & $15 \pm 3$ \\
\hline SMC5_004201 & $3.6 \pm 0.4$ & $6.6 \pm 1.0$ & $6.7 \pm 2.5$ & $55 \pm 6$ \\
\hline SMC5_004509 & $3.6 \pm 0.4$ & $6.5 \pm 0.5$ & $5.2 \pm 1.0$ & $55 \pm 6$ \\
\hline SMC5_004685 & $3.4 \pm 0.4$ & $5.8 \pm 0.5$ & $4.7 \pm 1.0$ & $71 \pm 6$ \\
\hline SMC5_004982 & $3.5 \pm 0.4$ & $6.6 \pm 0.5$ & $4.0 \pm 1.0$ & $46 \pm 6$ \\
\hline SMC5_005045 & $3.3 \pm 0.4$ & $6.1 \pm 0.5$ & $3.4 \pm 0.5$ & $51 \pm 6$ \\
\hline SMC5_008231 & $4.4 \pm 0.4$ & $11.1 \pm 1.0$ & $10.2 \pm 1.5$ & $21 \pm 3$ \\
\hline SMC5_009378 & $3.4 \pm 0.4$ & $5.9 \pm 0.5$ & $5.2 \pm 1.0$ & $70 \pm 6$ \\
\hline SMC5_011371 & $3.6 \pm 0.4$ & $8.7 \pm 0.5$ & $2.8 \pm 0.5$ & $3 \pm 1$ \\
\hline SMC5_011991 & $3.7 \pm 0.4$ & $7.0 \pm 0.5$ & $5.3 \pm 1.0$ & $45 \pm 6$ \\
\hline SMC5_012717 & $3.4 \pm 0.4$ & $6.0 \pm 0.5$ & $4.0 \pm 1.0$ & $60 \pm 6$ \\
\hline SMC5_012767 & $4.7 \pm 0.4$ & $16.4 \pm 1.0$ & $7.4 \pm 1.0$ & $10 \pm 3$ \\
\hline SMC5_013233 & $2.5 \pm 0.4$ & $3.3 \pm 0.5$ & $2.7 \pm 0.5$ & $218 \pm 10$ \\
\hline SMC5_013978 & $3.9 \pm 0.4$ & $7.7 \pm 0.5$ & $7.1 \pm 1.5$ & $39 \pm 3$ \\
\hline SMC5_014052 & $4.1 \pm 0.4$ & $8.9 \pm 0.5$ & $9.2 \pm 1.5$ & $29 \pm 3$ \\
\hline SMC5_014114 & $4.7 \pm 0.4$ & $14.0 \pm 1.0$ & $14.3 \pm 1.5$ & $15 \pm 3$ \\
\hline SMC5_014212 & $4.0 \pm 0.4$ & $8.4 \pm 1.0$ & $8.1 \pm 1.5$ & $33 \pm 3$ \\
\hline SMC5_014271 & $4.9 \pm 0.4$ & $19.8 \pm 2.0$ & $7.5 \pm 1.5$ & $7 \pm 1$ \\
\hline SMC5_014637 & $3.8 \pm 0.4$ & $7.1 \pm 0.5$ & $7.2 \pm 1.5$ & $44 \pm 3$ \\
\hline SMC5_014727 & $3.6 \pm 0.4$ & $7.0 \pm 0.5$ & $4.5 \pm 1.0$ & $41 \pm 3$ \\
\hline SMC5_014864 & $3.5 \pm 0.4$ & $6.1 \pm 0.5$ & $6.9 \pm 1.0$ & $65 \pm 6$ \\
\hline SMC5_014878 & $4.3 \pm 0.4$ & $11.0 \pm 1.0$ & $8.1 \pm 1.5$ & $22 \pm 3$ \\
\hline SMC5_015509 & $3.5 \pm 0.4$ & $7.5 \pm 0.5$ & $3.1 \pm 0.5$ & $21 \pm 3$ \\
\hline SMC5_015867 & $3.4 \pm 0.4$ & $6.2 \pm 0.5$ & $3.6 \pm 0.5$ & $50 \pm 6$ \\
\hline SMC5_016177 & $3.8 \pm 0.4$ & $8.6 \pm 0.5$ & $3.9 \pm 0.5$ & $21 \pm 3$ \\
\hline SMC5_016461 & $4.8 \pm 0.4$ & $14.9 \pm 1.0$ & $23.9 \pm 2.0$ & $13 \pm 3$ \\
\hline SMC5_016477 & $2.5 \pm 0.4$ & $3.0 \pm 0.5$ & $4.9 \pm 0.5$ & $301 \pm 10$ \\
\hline SMC5_016486 & $4.4 \pm 0.4$ & $13.4 \pm 1.0$ & $6.1 \pm 1.5$ & $13 \pm 3$ \\
\hline SMC5_016523 & $4.1 \pm 0.4$ & $9.5 \pm 0.5$ & $7.6 \pm 1.0$ & $27 \pm 3$ \\
\hline SMC5_016544 & $3.8 \pm 0.4$ & $8.4 \pm 1.0$ & $5.1 \pm 1.5$ & $30 \pm 3$ \\
\hline SMC5_016824 & $3.7 \pm 0.4$ & $7.0 \pm 0.5$ & $6.5 \pm 1.0$ & $46 \pm 6$ \\
\hline SMC5_017596 & $3.4 \pm 0.4$ & $6.0 \pm 0.5$ & $3.9 \pm 1.0$ & $58 \pm 6$ \\
\hline SMC5_018501 & $4.1 \pm 0.4$ & $10.0 \pm 0.5$ & $5.4 \pm 1.0$ & $22 \pm 3$ \\
\hline SMC5_020211 & $4.0 \pm 0.4$ & $9.0 \pm 1.0$ & $6.0 \pm 1.0$ & $28 \pm 3$ \\
\hline SMC5_021152 & $4.2 \pm 0.4$ & $10.0 \pm 1.0$ & $12.5 \pm 1.0$ & $25 \pm 3$ \\
\hline SMC5_021886 & $3.7 \pm 0.4$ & $8.5 \pm 1.0$ & $3.7 \pm 1.5$ & $21 \pm 3$ \\
\hline SMC5_022295 & $4.6 \pm 0.4$ & $13.2 \pm 0.5$ & $14.3 \pm 1.0$ & $16 \pm 3$ \\
\hline SMC5_022628 & $3.9 \pm 0.4$ & $9.6 \pm 1.0$ & $4.2 \pm 1.5$ & $18 \pm 3$ \\
\hline SMC5_022842 & $3.8 \pm 0.4$ & $9.2 \pm 1.0$ & $4.0 \pm 1.5$ & $19 \pm 3$ \\
\hline SMC5_023931 & $3.7 \pm 0.4$ & $7.5 \pm 0.5$ & $4.7 \pm 0.5$ & $37 \pm 3$ \\
\hline SMC5_025052 & $3.2 \pm 0.4$ & $4.9 \pm 0.5$ & $4.5 \pm 1.0$ & $91 \pm 6$ \\
\hline
\end{tabular}


Table 6. continued.

\begin{tabular}{|c|c|c|c|c|}
\hline Star & $\overline{\log \left(L / L_{\odot}\right)}$ & $\overline{M / M_{\odot}}$ & $\overline{R / R_{\odot}}$ & age Myears \\
\hline SMC5_025589 & $2.6 \pm 0.4$ & $4.0 \pm 0.5$ & $2.0 \pm 0.5$ & $57 \pm 6$ \\
\hline SMC5_025718 & $4.1 \pm 0.4$ & $8.9 \pm 0.5$ & $8.0 \pm 0.5$ & $29 \pm 3$ \\
\hline SMC5_025816 & $3.9 \pm 0.4$ & $7.9 \pm 1.0$ & $8.1 \pm 1.5$ & $38 \pm 3$ \\
\hline SMC5 025829 & $3.7 \pm 0.4$ & $7.1 \pm 0.5$ & $5.9 \pm 1.5$ & $44 \pm 6$ \\
\hline SMC5 026182 & $2.5 \pm 0.4$ & $3.7 \pm 0.5$ & $2.1 \pm 1.0$ & $119 \pm 6$ \\
\hline SMC5_026689 & - & - & - & - \\
\hline SMC5_028368 & $3.6 \pm 0.4$ & $7.0 \pm 0.5$ & $4.2 \pm 0.5$ & $38 \pm 3$ \\
\hline SMC5_036967 & $4.1 \pm 0.4$ & $8.8 \pm 0.5$ & $8.8 \pm 1.5$ & $31 \pm 3$ \\
\hline SMC5_037013 & $4.4 \pm 0.4$ & $11.0 \pm 1.0$ & $13.5 \pm 1.5$ & $22 \pm 3$ \\
\hline SMC5 037137 & $3.9 \pm 0.4$ & $7.9 \pm 1.0$ & $6.1 \pm 1.5$ & $38 \pm 3$ \\
\hline SMC5 037158 & $3.9 \pm 0.4$ & $9.5 \pm 0.5$ & $4.6 \pm 1.0$ & $21 \pm 3$ \\
\hline SMC5_037162 & $3.9 \pm 0.4$ & $8.1 \pm 0.5$ & $5.6 \pm 1.0$ & $35 \pm 3$ \\
\hline SMC5_038007 & $2.8 \pm 0.4$ & $4.2 \pm 0.5$ & $2.6 \pm 1.0$ & $97 \pm 6$ \\
\hline SMC5_038312 & $2.7 \pm 0.4$ & $3.5 \pm 0.5$ & $4.5 \pm 0.5$ & $217 \pm 10$ \\
\hline SMC5_038363 & $4.0 \pm 0.4$ & $9.3 \pm 0.5$ & $5.1 \pm 1.0$ & $24 \pm 3$ \\
\hline SMC5_041410 & $4.6 \pm 0.4$ & $13.7 \pm 1.0$ & $10.9 \pm 1.5$ & $15 \pm 3$ \\
\hline SMC5_043413 & $3.5 \pm 0.4$ & $6.7 \pm 0.5$ & $4.4 \pm 1.0$ & $47 \pm 3$ \\
\hline SMC5_044117 & $3.4 \pm 0.4$ & $6.4 \pm 0.5$ & $3.6 \pm 1.0$ & $45 \pm 3$ \\
\hline SMC5_044693 & $4.0 \pm 0.4$ & $8.5 \pm 0.5$ & $7.4 \pm 1.0$ & $33 \pm 3$ \\
\hline SMC5_044898 & $4.2 \pm 0.4$ & $10.3 \pm 1.0$ & $8.4 \pm 1.5$ & $24 \pm 3$ \\
\hline SMC5_045353 & $4.0 \pm 0.4$ & $8.7 \pm 1.0$ & $7.7 \pm 1.5$ & $31 \pm 3$ \\
\hline SMC5_045747 & $3.7 \pm 0.4$ & $7.0 \pm 0.5$ & $5.8 \pm 1.0$ & $46 \pm 3$ \\
\hline SMC5_046388 & $3.7 \pm 0.4$ & $7.6 \pm 0.5$ & $4.8 \pm 1.0$ & $37 \pm 3$ \\
\hline SMC5 046462 & $3.7 \pm 0.4$ & $7.6 \pm 0.5$ & $4.5 \pm 1.0$ & $34 \pm 3$ \\
\hline SMC5_047763 & $4.2 \pm 0.4$ & $9.0 \pm 0.5$ & $26.4 \pm 1.0$ & $30 \pm 3$ \\
\hline SMC5_048045 & $4.0 \pm 0.4$ & $8.5 \pm 0.5$ & $8.2 \pm 2.0$ & $33 \pm 3$ \\
\hline SMC5_048047 & $3.9 \pm 0.4$ & $8.0 \pm 0.5$ & $6.9 \pm 1.0$ & $37 \pm 3$ \\
\hline SMC5_048289 & $3.4 \pm 0.4$ & $6.2 \pm 0.5$ & $3.7 \pm 1.0$ & $53 \pm 6$ \\
\hline SMC5_049651 & $2.3 \pm 0.4$ & $3.1 \pm 0.5$ & $2.1 \pm 0.5$ & $210 \pm 10$ \\
\hline SMC5_049746 & $3.6 \pm 0.4$ & $7.4 \pm 0.5$ & $4.4 \pm 1.0$ & $37 \pm 3$ \\
\hline SMC5_049780 & $4.1 \pm 0.4$ & $10.3 \pm 1.0$ & $5.4 \pm 1.0$ & $20 \pm 3$ \\
\hline SMC5_049996 & $3.7 \pm 0.4$ & $7.7 \pm 1.0$ & $4.5 \pm 1.5$ & $34 \pm 3$ \\
\hline SMC5_051315 & $2.4 \pm 0.4$ & $3.1 \pm 0.5$ & $2.7 \pm 0.5$ & $254 \pm 10$ \\
\hline SMC5_052688 & $3.8 \pm 0.4$ & $7.4 \pm 0.5$ & $6.6 \pm 1.5$ & $42 \pm 6$ \\
\hline SMC5_053267 & $3.8 \pm 0.4$ & $8.5 \pm 0.5$ & $4.1 \pm 1.0$ & $24 \pm 3$ \\
\hline SMC5_053756 & $3.6 \pm 0.4$ & $6.7 \pm 0.5$ & $4.7 \pm 1.0$ & $48 \pm 6$ \\
\hline SMC5_055592 & $3.4 \pm 0.4$ & $5.7 \pm 0.5$ & $5.5 \pm 1.0$ & $74 \pm 6$ \\
\hline SMC5 061950 & $2.9 \pm 0.4$ & $4.0 \pm 0.5$ & $4.4 \pm 1.0$ & $142 \pm 6$ \\
\hline SMC5 064327 & $4.2 \pm 0.4$ & $9.7 \pm 1.0$ & $11.3 \pm 2.0$ & $26 \pm 3$ \\
\hline SMC5_064576 & $3.0 \pm 0.4$ & $5.6 \pm 0.5$ & $2.2 \pm 0.5$ & $9 \pm 1$ \\
\hline SMC5_064745 & $5.0 \pm 0.4$ & $21.8 \pm 1.5$ & $9.1 \pm 1.5$ & $7 \pm 1$ \\
\hline SMC5_064832 & $3.4 \pm 0.4$ & $5.5 \pm 0.5$ & $5.6 \pm 1.0$ & $78 \pm 6$ \\
\hline SMC5_065055 & $4.8 \pm 0.4$ & $15.3 \pm 1.0$ & $11.6 \pm 1.5$ & $13 \pm 3$ \\
\hline SMC5_065746 & $3.4 \pm 0.4$ & $7.9 \pm 0.5$ & $2.6 \pm 0.5$ & $3 \pm 1$ \\
\hline SMC5_066754 & $3.6 \pm 0.4$ & $6.5 \pm 0.5$ & $5.1 \pm 1.0$ & $57 \pm 6$ \\
\hline SMC5 067333 & $3.3 \pm 0.4$ & $6.0 \pm 0.5$ & $3.5 \pm 1.0$ & $54 \pm 6$ \\
\hline SMC5_073581 & $3.7 \pm 0.4$ & $6.8 \pm 0.5$ & $6.7 \pm 1.0$ & $50 \pm 6$ \\
\hline SMC5_073594 & $3.6 \pm 0.4$ & $6.3 \pm 0.5$ & $5.6 \pm 1.0$ & $60 \pm 6$ \\
\hline SMC5_074402 & $3.9 \pm 0.4$ & $8.2 \pm 1.0$ & $6.4 \pm 1.5$ & $35 \pm 3$ \\
\hline SMC5_074471 & $4.1 \pm 0.4$ & $9.1 \pm 1.0$ & $9.5 \pm 1.5$ & $28 \pm 3$ \\
\hline SMC5_075061 & $2.9 \pm 0.4$ & $4.3 \pm 0.5$ & $3.8 \pm 0.5$ & $127 \pm 6$ \\
\hline SMC5 075360 & $3.6 \pm 0.4$ & $6.5 \pm 0.5$ & $5.0 \pm 1.0$ & $56 \pm 6$ \\
\hline SMC5_078338 & $3.8 \pm 0.4$ & $7.3 \pm 0.5$ & $7.2 \pm 1.0$ & $43 \pm 3$ \\
\hline SMC5_078440 & $4.0 \pm 0.4$ & $8.7 \pm 1.0$ & $7.9 \pm 2.0$ & $31 \pm 3$ \\
\hline SMC5_078928 & $3.5 \pm 0.4$ & $6.3 \pm 0.5$ & $5.0 \pm 1.0$ & $60 \pm 6$ \\
\hline SMC5_080910 & $3.4 \pm 0.4$ & $6.4 \pm 0.5$ & $3.8 \pm 1.0$ & $48 \pm 6$ \\
\hline SMC5_081260 & $2.3 \pm 0.4$ & $3.3 \pm 0.5$ & $2.0 \pm 1.0$ & $159 \pm 6$ \\
\hline SMC5_082042 & $3.9 \pm 0.4$ & $7.8 \pm 1.0$ & $6.7 \pm 2.0$ & $38 \pm 3$ \\
\hline SMC5_082202 & $3.3 \pm 0.4$ & $5.0 \pm 0.5$ & $5.4 \pm 1.0$ & $88 \pm 6$ \\
\hline SMC5_082543 & $3.7 \pm 0.4$ & $7.9 \pm 0.5$ & $3.7 \pm 1.0$ & $26 \pm 3$ \\
\hline
\end{tabular}


Table 6. continued.

\begin{tabular}{ccccc}
\hline \hline Star & $\log \left(L / L_{\odot}\right)$ & $M / M_{\odot}$ & $R / R_{\odot}$ & age Myears \\
\hline SMC5_082819 & $5.0 \pm 0.4$ & $18.4 \pm 1.0$ & $20.3 \pm 2.5$ & $11 \pm 3$ \\
SMC5_082941 & $3.9 \pm 0.4$ & $7.9 \pm 1.0$ & $6.8 \pm 1.5$ & $38 \pm 3$ \\
SMC5_083491 & $4.1 \pm 0.4$ & $8.9 \pm 1.0$ & $8.1 \pm 2.0$ & $30 \pm 3$ \\
SMC5_085503 & $3.7 \pm 0.4$ & $6.5 \pm 0.5$ & $8.5 \pm 1.5$ & $56 \pm 6$ \\
SMC5_086200 & $3.3 \pm 0.4$ & $5.7 \pm 0.5$ & $3.5 \pm 1.0$ & $61 \pm 6$ \\
SMC5_086251 & $3.4 \pm 0.4$ & $6.3 \pm 0.5$ & $3.7 \pm 1.0$ & $50 \pm 6$ \\
SMC5_086581 & $2.5 \pm 0.4$ & $3.6 \pm 0.5$ & $2.5 \pm 1.0$ & $167 \pm 6$ \\
SMC5_086890 & $3.8 \pm 0.4$ & $8.9 \pm 0.5$ & $4.0 \pm 1.0$ & $21 \pm 3$ \\
SMC5_086983 & $3.8 \pm 0.4$ & $8.1 \pm 0.5$ & $4.5 \pm 1.0$ & $30 \pm 3$ \\
SMC5_087004 & $3.5 \pm 0.4$ & $8.0 \pm 0.5$ & $2.9 \pm 0.5$ & $11 \pm 3$ \\
SMC5_090914 & $3.7 \pm 0.4$ & $6.9 \pm 0.5$ & $7.4 \pm 1.5$ & $47 \pm 6$ \\
SMC5_190576 & $5.5 \pm 0.4$ & $30.6 \pm 2.5$ & $17.4 \pm 2.0$ & $6 \pm 3$ \\
\hline
\end{tabular}

Table 8. Comparison of the rotational velocities for B and Be stars in fields and clusters in the SMC, LMC, and in the MW following statistical tests. Column 3 gives the result of the Student's t-test, Col. 4 gives the $\alpha$ coefficient, Col. 5 gives the probability of difference, and the last column comments on the result following the convention. The comment "limit" means that the number of stars in samples is low and the value of mean $V \sin i$ may be affected by the distribution of the inclination angles.

\begin{tabular}{cccccc}
\hline \hline & Comparison & $T_{n 1, n 2}$ & $\alpha$ & probability & comments \\
\hline Field & SMC Be / LMC Be & 6.08 & 0.1 & $80-90 \%$ & slight difference \\
Field & SMC Be / MW Be Chauville et al (2001) & 15.4 & 0.02 & $95-98 \%$ & significant difference \\
Field & SMC Be / MW Be Glebocki et al. (2000) & & & & \\
Field & SMC Be / MW Be Yudin (2001) & & & & \\
\hline Field & SMC B / LMC B & 16.78 & 0.02 & $95-98 \%$ & significant difference \\
Field & SMC B / MW B Glebocki et al. (2000) & 29.38 & 0.02 & $95-98 \%$ & significant difference \\
Field & SMC B / MW B Levato et al. (2004) & & & & \\
\hline Cluster & SMC Be / LMC Be & 0.17 & 0.5 & $10-50 \%$ & no difference \\
Cluster & SMC Be / MW Be WEBDA log $(t)<7$ & 4.29 & 0.1 & $80-90 \%$ & limit, slight difference \\
Cluster & SMC Be / MW Be WEBDA $\log (t)>7$ & 7.69 & 0.05 & $90-95 \%$ & significant difference \\
\hline Cluster & SMC B / LMC B & 1.71 & 0.30 & $50-7-0 \%$ & limit, no difference \\
Cluster & SMC B / MW B WEBDA log $(t)<7$ & 5.45 & 0.1 & $80-90 \%$ & slight difference \\
Cluster & SMC B / MW B WEBDA $\log (t)>7$ & 1.65 & 0.3 & $50-70 \%$ & no difference \\
\hline & SMC Be field / SMC Be clusters & 6.12 & 0.1 & $80-90 \%$ & slight difference \\
& SMC B field / SMC B clusters & 0.596 & 0.5 & $50 \%$ & no difference \\
\hline
\end{tabular}

Table 11. Comparison of the rotational velocities for Be stars in the SMC, LMC, and in the MW, which have similar age and masses by the Student's t-test. The second column gives the $\alpha$ coefficient, the fourth column gives the probability of difference, and the last column comments on the result following the convention. The comment "limit" means that the number of stars in samples is low and the value of mean $V$ sin $i$ may be affected by the distribution of the inclination angles.

\begin{tabular}{|c|c|c|c|}
\hline Comparison & $\overline{\alpha \alpha}$ & probability & comments \\
\hline $\mathrm{SMC} \mathrm{Be} \mathrm{/} \mathrm{MW} \mathrm{Be}$ & $\begin{array}{c}2 \leq M<5 M_{\odot} \\
0.085\end{array}$ & $91.5 \%$ & slight difference \\
\hline $\begin{array}{l}\text { SMC Be / LMC Be } \\
\text { SMC Be / MW Be } \\
\text { LMC Be / MW Be }\end{array}$ & $\begin{array}{c}5 \leq M<10 M_{\odot} \\
0.298 \\
0.0001 \\
0.005\end{array}$ & $\begin{array}{c}70.2 \% \\
99.99 \% \\
99.5 \%\end{array}$ & $\begin{array}{c}\text { no difference } \\
\text { extremely significant difference } \\
\text { highly significant difference }\end{array}$ \\
\hline $\begin{array}{l}\text { SMC Be / LMC Be } \\
\text { SMC Be / MW Be } \\
\text { LMC Be / MW Be }\end{array}$ & $\begin{array}{c}10 \leq M<12 M_{\odot} \\
0.024 \\
0.013 \\
0.25\end{array}$ & $\begin{array}{l}97.7 \% \\
98.7 \% \\
75.1 \%\end{array}$ & $\begin{array}{c}\text { significant difference } \\
\text { limit, highly significant difference } \\
\text { limit, no difference }\end{array}$ \\
\hline $\mathrm{SMC} \mathrm{Be} \mathrm{/} \mathrm{LMC} \mathrm{Be}$ & $\begin{array}{c}12 \leq M<18 M_{\odot} \\
0.008\end{array}$ & $99.2 \%$ & highly significant difference \\
\hline
\end{tabular}

